The final version of this paper was published in The International Journal of Robotics Research, 29(7), June 2010, pp. 855-896, by SAGE Publications Ltd, http://online.sagepub.com. (C)Michael Erdmann

\title{
On the Topology of Discrete Strategies*
}

\author{
Michael Erdmann \\ School of Computer Science \\ Carnegie Mellon University \\ me@cs.cmu.edu
}

December 2009

\begin{abstract}
This paper explores a topological perspective of planning in the presence of uncertainty, focusing on tasks specified by goal states in discrete spaces. The paper introduces strategy complexes. A strategy complex is the collection of all plans for attaining all goals in a given space. Plans are like jigsaw pieces. Understanding how the pieces fit together in a strategy complex reveals structure. That structure characterizes the inherent capabilities of an uncertain system. By adjusting the jigsaw pieces in a design loop, one can build systems with desired competencies.

The paper draws on representations from combinatorial topology, Markov chains, and polyhedral cones. Triangulating between these three perspectives produces a topological language for describing concisely the capabilities of uncertain systems, analogous to concepts of reachability and controllability in other disciplines. The major nouns in this language are topological spaces.

Three key theorems (numbered 1, 11, 20 in the paper) illustrate the sentences in this language: (a) Goal Attainability: There exists a strategy for attaining a particular goal from anywhere in a system if and only if the strategy complex of a slightly modified system is homotopic to a sphere. (b) Full Controllability: A system can move between any two states despite control uncertainty precisely when its strategy complex is homotopic to a sphere of dimension two less than the number of states. (c) General Structure: Any system's strategy complex is homotopic to the product of a spherical part, modeling full controllability on subspaces, and a general part, modeling adversarial capabilities.

The paper contains a number of additional results required as stepping stones, along with many examples. The paper provides algorithms for computing the key structures described. Finally, the paper shows that some interesting questions are hard. For instance, it is $N P$-complete to determine the most precisely attainable goal of a system with perfect sensing but uncertain control.
\end{abstract}

This work was sponsored by DARPA under contract HR0011-07-1-0002. This work does not necessarily reflect the position or the policy of the U.S. Government. No official endorsement should be inferred.

${ }^{*}$ A much abbreviated version of this paper appeared as [28]. 


\section{Contents}

1 Introduction $\quad 4$

1.1 Planning Manipulation Strategies with Uncertainty . . . . . . . . . . . . . . 4

1.2 Understanding System Capabilities . . . . . . . . . . . . . . . . . . 4

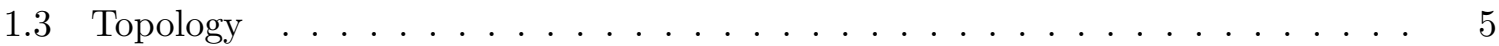

1.4 Result Flavor . . . . . . . . . . . . . . . . . . . . . . . 5

1.5 Spheres as Topological Descriptors of Task Solvability . . . . . . . . . . . 6

1.6 Contributions . . . . . . . . . . . . . . . . . . 6

1.7 An Underlying Motivation . . . . . . . . . . . . . . . . 7

1.8 Broader Context . . . . . . . . . . . . . . . . . . 7

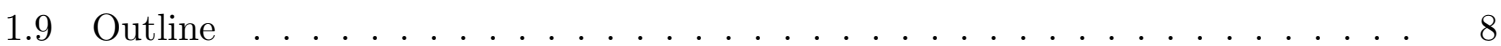

2 An Example: Nondeterminism, Cycles, and Strategies 9

3 Nondeterministic Graphs and Strategy Complexes $\quad 11$

3.1 Basic Definitions . . . . . . . . . . . . . . . . . . . 11

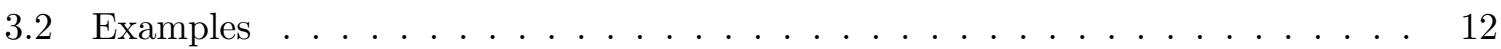

4 Loopback Graphs and Complexes $\quad 15$

5 Topological Tools and Homotopy Equivalence $\quad 17$

5.1 Deformation Retractions . . . . . . . . . . . . . . . . . . . . 18

5.2 Collapsibility and Contractibility . . . . . . . . . . . . . 18

5.3 The Nerve Lemma . . . . . . . . . . . . . . . . . . . . . . . . . . . 19

5.4 The Quillen Fiber Lemma . . . . . . . . . . . . . . . . . . . . 19

5.5 Homotopy Interpretation . . . . . . . . . . . . . . . . . . 20

6 Stochastic Graphs and Strategy Complexes 20

6.1 Stochastic Actions and Graphs . . . . . . . . . . . . . . . . . . . . 20

6.2 Stochastic Acyclicity . . . . . . . . . . . . . . . . . . . 21

6.3 Stochastic Strategy Complexes . . . . . . . . . . . . . . . . 23

7 Covering Sets $\quad \mathbf{2 5}$

7.1 Homogeneous Covering Sets . . . . . . . . . . . . . . . . . . 25

7.2 Affine Covering Sets . . . . . . . . . . . . . . . . 25

7.3 Inferring Topology from Covering Sets . . . . . . . . . . . . . . . 26

8 Controllability of Motions in Stochastic Graphs 30

8.1 Connectivity: Covers and Chains . . . . . . . . . . . . . . . . . 31

8.2 Characterizing Controllability with Spheres . . . . . . . . . . . . . 34

9 Topology as a Design Tool: An Example $\quad 36$

9.1 How Many Design Scenarios? . . . . . . . . . . . . . . . . . . . . 36

9.2 Tuning Convergence Times and Designing System Capabilities . . . . . . . . 37 
10 Duality $\quad 41$

10.1 Start Region Contractibility . . . . . . . . . . . . . . . . . . 41

10.2 Source Complex. . . . . . . . . . . . . . . . . . . . 42

10.3 Contractibility Characterization of Goal Attainability . . . . . . . . . . . . 43

10.4 The Dual Complex . . . . . . . . . . . . . . . . . . . . . . . . . . . . . . . . . .

10.5 Duality in Design . . . . . . . . . . . . . . . . . . . 44

11 Modularity $\quad 46$

11.1 Graph Union . . . . . . . . . . . . . . . . . . . . . . 46 46

11.2 Testing Acyclicity _. . . . . . . . . . . . . . . . . 47

11.3 Simplification via Strongly Controllable Subspaces . . . . . . . . . . . . . . 48

11.4 An Example (Air Travel During Thunderstorm Season) . . . . . . . . . . . . 52

12 Algorithms $\quad 55$

13 Realizability $\quad 59$

14 Hardness $\quad \mathbf{6 0}$

14.1 The Difficulty of Determining a System's Precision . . . . . . . . . . . . . . . 60

14.2 Small Realization is Uncomputable . . . . . . . . . . . . . . . . . . . 61

14.3 Recognizing Repercussions is Uncomputable . . . . . . . . . . . . . . . . . 62

15 Topological Thinking $\quad 63$

15.1 Topology Precompiles an Existence Argument . . . . . . . . . . . . . . . 63

15.2 Topological Analysis of Adversity . . . . . . . . . . . . . . . . . . 64

15.3 Topological Thinking in Partially Observable Spaces . . . . . . . . . . . . . . 66

15.3.1 Inferring Task Unsolvability From Duality . . . . . . . . . . . . . . 67

15.3.2 Hypothesis-Testing and Sphere Suspension . . . . . . . . . . . . 69

$\begin{array}{ll}16 \text { Conclusions } & \mathbf{7 1}\end{array}$

16.1 Summary . . . . . . . . . . . . . . . . . . . . 71

16.2 Other Results . . . . . . . . . . . . . . . . . . . . 71

16.3 Open Questions . . . . . . . . . . . . . . . . . . . 71

17 Acknowledgments $\quad 72$

$\begin{array}{ll}\text { List of Primary Symbols } & 73\end{array}$

$\begin{array}{ll}\text { List of Lemmas and Theorems } & \mathbf{7 4}\end{array}$

$\begin{array}{ll}\text { List of Algorithms } & 75\end{array}$

$\begin{array}{ll}\text { List of Key Definitions } & 75\end{array}$

$\begin{array}{ll}\text { List of Figures } & 76\end{array}$

$\begin{array}{ll}\text { References } & \mathbf{7 7}\end{array}$ 


\section{Introduction}

\subsection{Planning Manipulation Strategies with Uncertainty}

Two themes pervade the last four decades of research in robotic manipulation. The first is the difficulty of uncertainty, the second is a solution by discrete modeling. The jamming diagram appearing in Whitney's classic paper on peg-in-hole assembly [77], grounded in extensive work at the C.S. Draper Laboratory [62] in the 1970s, illustrates both themes: (i) The precise location and motion of the peg are uncertain. (ii) The contact state of the peg naturally discretizes the assembly space. By analyzing this discretized space, [77] produces an automatic strategy for assembly that is robust in the presence of uncertainty.

As this early example demonstrates, discretization appears in manipulation research to simplify, but not artificially so. Instead, the mechanics of a problem often generate natural discrete states even when the initial rendering of the problem is continuous. Contact modes, describing which features of multiple objects are in contact and how are they sliding relative to each other, are particularly common methods of discretization. As further illustration, the cooperative manipulation strategies of [27] are based on discrete states representing regions of configuration space over which the frictional contact mechanics of the robot palms and the part are invariant. Discrete states may also capture higher-order information, perhaps modeling sensing uncertainty. For instance, in the part orienters of $[29,72,37,30]$, the discrete states considered by the motion planners were sets of underlying contact states of the parts being oriented. In this manner, sensing uncertainty itself contributes to the definition of state $[5,54]$.

Delineating relevant states is only part of discrete modeling. One must also describe transitions between states, with the aim of synthesizing robust manipulation strategies. Anyone who has ever programmed a robot manipulator quickly learns never to expect a particular grasping operation or a particular assembly trajectory to succeed. Instead, transitions between states are generally uncertain, due both to underlying control uncertainty and, in the discrete case, to approximation effects. As a result, the discrete modeling of a manipulation problem does not define a standard directed graph, but rather a nondeterministic or stochastic graph, in which commanded motions may have any one of several possible outcomes. Consequently, one must program robots by thinking in terms of sets of possible motions not individual trajectories. The preimage methodology of [56] emphasizes this point and shows how to generate robust strategies in a fairly general, continuous, setting. In the discrete setting, that methodology is akin to Bellman-style backchaining [4]. Recent manipulation results [42, 43] demonstrate the utility of these ideas in stochastic settings.

\section{$1.2 \quad$ Understanding System Capabilities}

The description of planning above is highly operational. One can almost turn a crank: Given a manipulation task, create a discrete representation of the task based on the task mechanics and uncertainties, then backchain from the task goal. Missing is an understanding of the full capabilities of such a discrete representation. Operationally, one can report whether a planner found a plan to perform some task, but one does not understand intrinsically when there should be a plan and what failure to find a plan really means. It would be good to understand what the remedy for a failure might be, for instance, more precise sensing, more precise control, a change in the task goal, or perhaps even a remodeling of the task description and discretization. 
Our field does not even have an adequate language to describe the issues. In control theory, terms such as "controllable", "unstable", and "limit cycle" are common; in graph theory, "strongly connected", "source" and "sink"; in Markov chains, "recurrent class", "transient" and "absorbing". In different languages and in different settings, these terms describe similar concepts, significant for characterizing system capabilities.

Such terms also offer standards by which to design systems. One learns how to create or destroy limit cycles in control theory, for example. One understands that a finite Markov chain cannot consist exclusively of transient states, whereas an infinite one can. All effective engineering fields have such concise descriptions of capabilities. For instance, to pick an example very different from the previous ones, in computer language theory, one learns that certain context-free grammars are easily compilable while others are not.

Planning in the presence of uncertainty has no such concise descriptions of capabilities and thus no concise design standards.

\subsection{Topology}

A thesis of this paper is that topology provides a language for concise descriptions of system capabilities. In order to develop that language, we follow a tack common in engineering disciplines: Given a method for solving any particular problem operationally, one should attempt to classify problems and solutions generally, for instance, by observing similarities in problems and exploiting commonalities of solution. In the case of planning for uncertain systems, a plan seldom exists in isolation; instead it is part of an ensemble of interconnected strategies for accomplishing an ensemble of interconnected goals in some system.

Topology is good at extracting structure from such an interconnected patchwork. That structure constitutes our language. Moreover, this language, as we will see, has two desirable traits:

1. The topological descriptors abstract away details of particular trajectories, focusing instead on overall system capabilities.

2. The topological descriptors are consistent with existing terminology for standard directed graphs and Markov chains, while generalizing both.

Our search specifically for a topological language to describe system capabilities was motivated by Robert Ghrist's technology transfer between topology and robotics [13, 36, 34, 35], by Steven LaValle's work on information spaces [38, 65, 74, 73], and by workshops on topology and robotics organized by Michael Farber at ETH Zürich in 2003 and 2006 [57].

\subsection{Result Flavor}

An analogy for understanding the relationship of topology to planning might be the relationship of linear algebra to vectors written with specific coordinates. Linear algebra provides abstract techniques for representing and manipulating vectors, independent of coordinates. Anything one can do with matrix and vector notation one can do as well by writing out arrays of coordinates and manipulating the coordinates directly. Indeed, ultimately computations in a computer must work with numbers in some coordinate system. However, the numbers are like trees that obscure the forest. By thinking instead at the abstract level of linear subspaces, kernels, eigenvectors, and so forth, one can recognize fundamental structure. How easy it is 
to say that a high-dimensional positive definite system always decomposes into orthogonal one-dimensional systems; how cumbersome it would be to convey that truth by numbers or coordinates alone.

This perspective has precedent in other areas of computer science, such as the pioneering work of Herlihy on asynchronous computation. For instance, [40] shows that an asynchronous decision task has a wait-free protocol if and only if there exists a certain color-preserving continuous function between two chromatic simplicial complexes determined by the task. In other words, a computational problem is equivalent to a topological problem. The simplicial complexes reflect the structure of the input and output spaces of the task. The continuous function is a topological constraint between those spaces and thus a constraint on solvability of the decision task.

In topological robotics, Farber initiated a line of work to describe the topological complexity of motion planning problems on a space in terms of the cohomology of that space [31]. This complexity reflects the discontinuities inherent to any controller that maps start and goal configurations of a robot to trajectories between those configurations.

An early example demonstrating the applicability specifically of algebraic topology to robotics, and indeed manipulation, appears in the work of Hopcroft and Wilfong [41]. They use the Mayer-Vietoris sequence on the zeroth and first homology groups to study contact connectivity. A key result: Under certain conditions on the structure of configuration space, if there is a motion of two objects starting and ending with the objects in contact, then there exists a motion throughout which the objects remain in contact.

\subsection{Spheres as Topological Descriptors of Task Solvability}

The current paper focuses on tasks that may be specified by goal states in some nondeterministic or stochastic graph; the task is to attain some goal state starting from anywhere within the graph. A key result (Theorem 1) shows that such a task has a guaranteed solution if and only if a certain simplicial complex associated with the task is homotopic to a sphere of a certain dimension. This special result leads to a general graph controllability theorem (Theorem 11) that characterizes the ability of a system to achieve any goal despite control uncertainty, again in terms of the existence of a certain sphere. Spheres and contractible spaces are much like the linear subspaces and trivial kernels in our earlier analogy.

These results are motivated by similar results describing the structure of complete directed graphs [8] and strongly connected directed graphs [44]. Indeed, our proof techniques build on the foundations of those two papers. Of additional interest is the extensive analysis in [47].

It is worth noting that the domain of nondeterministic graphs is much richer than that of directed graphs. It turns out (Section 13) that every finite simplicial complex can be realized via some nondeterministic graph. This correspondence further underscores the natural connection of topology to planning in the presence of uncertainty.

\subsection{Contributions}

The primary contribution of this paper is the previously unseen structure it reveals in planning problems. The paper shows how the details of a nondeterministic or stochastic graph may be abstracted away, leaving a purely topological description of the task: One may reason about task solvability by thinking in terms of spheres and contractible spaces. 
A second contribution is the introduction of particular tools, such as strategy complexes. A single strategy on a graph is an eventually convergent nondeterministic control law for moving within some portion of the graph. A strategy complex consists of all possible strategies on a graph. Strategy complexes can be useful for reasoning about alternate strategies that a system might need if a selected strategy for accomplishing a task fails unexpectedly.

Third, although largely beyond the scope of this paper, a stream of ancillary results flows from our characterization of task solvability, among them: (i) One can answer purely topologically the question whether a collection of actions is essential to solving a task. (ii) One can recast various questions about tasks into measurements by the Euler characteristic. (iii) One discovers that all strategies for accomplishing a goal in a nondeterministic graph must overlap a particular strategy found by backchaining. (iv) One observes that the number of strategies for attaining a single goal state is either zero or odd (this is the topological equivalent of Yogi Berra's direction: "When you come to a fork in the road, take it.").

We anticipate yet more structure will be discovered in planning problems via the lens of topology. Indeed, every key topological idea we have looked at thus far has had some significant meaning when recast in a planning context. The Combinatorial Alexander Dual discussed in Section 10 is a good example.

\subsection{An Underlying Motivation}

A longstanding motivation for our research has been a desire to understand system capabilities and the interplay of sensing and action. That motivation is rooted in a long line of work on motion planning with uncertainty from the 1980s and 1990s $[9,59,60,22,10,14,12,72,15$, $16,53,33,55,11]$ as well as related work on understanding information invariants, such as natural tradeoffs between sensing and action [70, 21, 18, 2, 19, 52, 26, 17, 45, 46]. The tools for characterizing system capabilities discussed in Sections 9 and 10 provide a concise topological language for making such comparisons.

\subsection{Broader Context}

Applying the ideas in this paper to robotics problems requires front-end work: one needs to cast a robot task as a motion planning problem within some graph whose transitions are (potentially) uncertain. Much of robotics research over the past three decades has been devoted precisely to such reductions. We will not review those techniques here, but point to two excellent books: [53] and [54]. For instance, in manipulation, one generally needs to describe the geometry and mechanics of contact, including dealing with issues such as friction, deformation, and so forth. In many instances, these analyses are fairly local. One then is left with the task of combining the local information to form global motion planners. The current paper presents a topological perspective of that second phase.

The topological perspective likely has broader applications than the motivating tasks of robotic manipulation. Section 11.4 provides a small example. We hope that the techniques in this paper will be useful for analyzing planning and decision-making problems in a wide variety of domains, namely those with two modular phases: a front-end discretization phase, followed by a planning phase. In particular, methods such as those discussed in Sections 9-15 should be useful not only for planning but for analyzing and fine-tuning the details of the front-end phase. Potentially relevant additional domains include decisional architecture design, temporal 
reasoning, and hybrid control systems.

\subsection{Outline}

Our basic approach is to replace the combinatorial structure of a discrete uncertain graph with the topology of a collection of open sets in Euclidean space. This technique is well-established for standard directed graphs $[8,44]$. We show how to generalize the approach to uncertain systems. In particular, convergence results for Markov chains appear as intersection properties of certain affine open cones.

The resulting topology characterizes system capabilities. We indicate how to use such characterizations for designing desired systems, for analyzing existing systems, and for general reasoning about motion with uncertainty.

- Section 1 discusses core approaches to planning manipulation strategies and how these have motivated this paper's topological inquiry.

- Section 2 provides intuition for understanding nondeterministic graphs and their strategy complexes, via a series of examples.

- Section 3 formally introduces nondeterministic graphs and their strategy complexes.

- Section 4 introduces loopback graphs and complexes, using these to characterize task solvability by the existence of topological spheres.

- Section 5 provides background on the topological tools appearing in this paper.

- Section 6 adds stochastic actions to the mix, now permitting both nondeterminism and stochasticity in a graph.

- Section 7 develops a connection between the combinatorial structure of plans and certain conical open sets in Euclidean space, useful for measuring a strategy's convergence times.

- Section 8 characterizes full controllability on a graph, despite control uncertainty, in terms of the existence of a sphere of dimension two less than the number of states in the graph.

- Section 9 explores methods for designing systems using the topological tools developed thus far.

- Section 10 dualizes the earlier spherical characterizations, leading both to contractibility characterizations and two new simplicial complexes: the source complex and its dual complex. The source complex consists of all start regions of convergent plans, the dual complex consists of all potentially unattainable goals.

- Section 11 discusses internal structure: how to combine graphs, how to simplify graphs. An implemented example illustrates the ideas.

- Section 12 contains algorithms to implement the core ideas of the paper.

- Section 13 proves two realizability theorems, establishing two different ways in which nondeterministic graphs are just like finite simplicial complexes. 
- Section 14 shows that ascertaining an uncertain system's precision is $N P$-complete. The section also shows that two natural questions one might ask of graphs are undecidable, based on the undecidability of recognizing contractibility.

- Section 15 discusses several different ways in which topological thinking illuminates planning problems.

\section{An Example: Nondeterminism, Cycles, and Strategies}
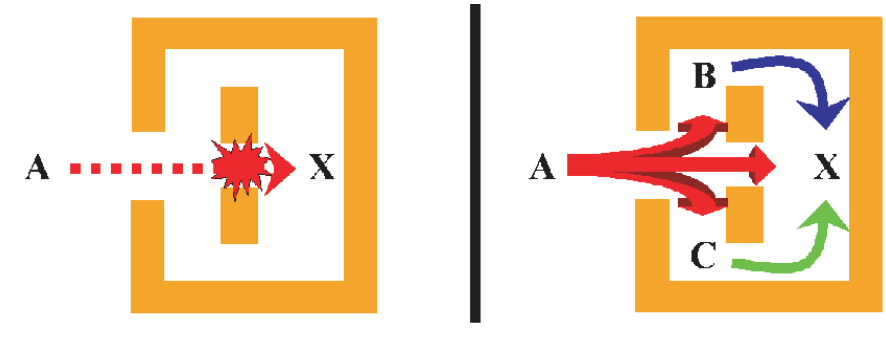

Figure 1: Direct access might be blocked.
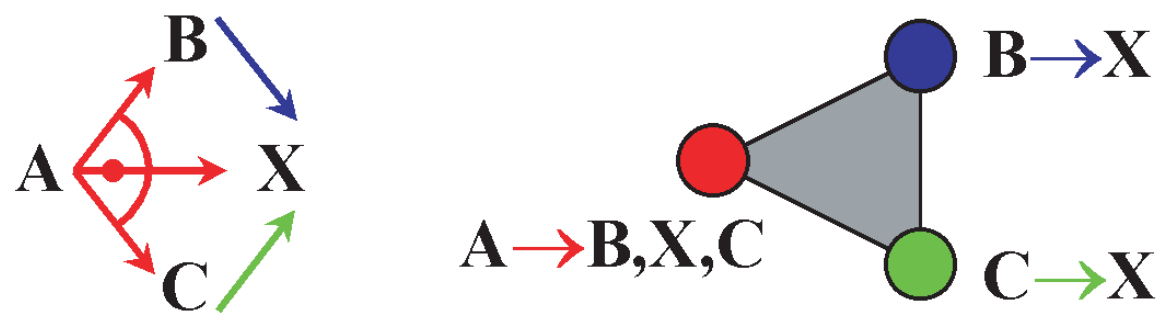

Figure 2: Nondeterministic graph and associated strategy complex for the motions of Fig. 1.

Legend: Figures in this paper depict nondeterministic actions in two ways: In a graph, as directed edges tied together by a circular arc, along with a "dot" inside the arc. In a strategy complex, by multiple outcomes to the right of an "arrow" $(\rightarrow)$.

Imagine an ambulance rescue in an old complicated city, perhaps after an earthquake. There are many opportunities for nondeterminism: Entries into the city might be blocked, maps might be wrong, navigation might lead to circular paths. Let us focus on the final step in which the ambulance medics must pass through a narrow opening to reach their patient, as in Fig. 1. Something might go wrong, a collapse of some sort, forcing the rescuers to either side. Let us suppose the rescuers may then take additional steps around buildings bounding the original narrow opening to reach their patient.

We can model this scenario using the graph in the left panel of Fig. 2. The action to move from A (ambulance) to $\mathrm{X}$ (patient) might nondeterministically lead to $\mathrm{X}$ but perhaps also to $\mathrm{B}$ or $\mathrm{C}$, depending on whether and how a collapse occurs on the direct path from A to $\mathrm{X}$. In the example, there then are deterministic actions from either B or C to X. Such a graph is essentially a compressed AND/OR graph [3]. 
We can now represent the strategy (or control law or plan) just described as a solid triangle (right panel of Fig. 2). The vertices of the triangle are the individual actions to be executed at any particular location during the rescue operation. So, for instance, the strategy says "When at location $\mathrm{B}$, execute the action $\mathrm{B} \rightarrow \mathrm{X}$."
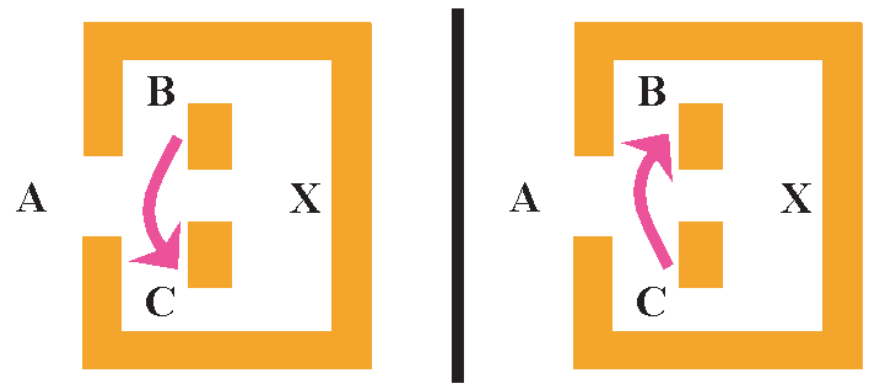

Figure 3: Actions that move between the far locations B and C.

Perhaps it is also possible to move from B to C, as in Fig. 3, and vice-versa. We can augment our graph to include these actions (left panel of Fig. 4)
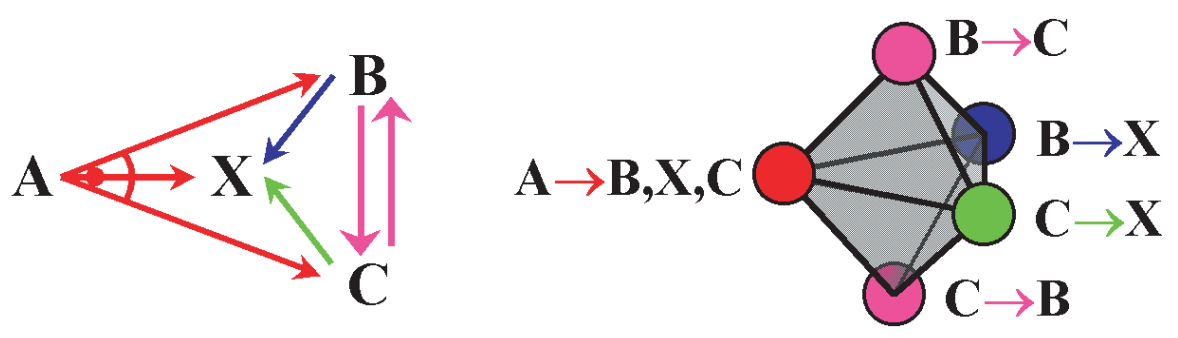

Figure 4: With additional (potentially cycle-inducing) actions, the strategy complex now contains two solid tetrahedra.

Once we have the additional actions $\mathrm{B} \rightarrow \mathrm{C}$ and $\mathrm{C} \rightarrow \mathrm{B}$, we have to be careful not to include both in a strategy. Otherwise, the rescuers (who could be robots, not humans) might cycle forever between B and C, never reaching their patient at X. Instead, we recognize that there now exist several distinct strategies for reaching $\mathrm{X}$. These can be represented by the two solid tetrahedra in the right panel of Fig. 4. Observe that the two tetrahedra intersect in a triangle that is our original triangle from Fig. 2, but there is no simplex that simultaneously includes the two actions $\mathrm{B} \rightarrow \mathrm{C}$ and $\mathrm{C} \rightarrow \mathrm{B}$.

Each tetrahedron represents a strategy (or control law or plan) consisting of actions that may be executed without accidentally creating cycles in the graph. Each triangle or edge or vertex of one of these tetrahedra, formed from a subset of the actions comprising the tetrahedron, also represents some strategy (perhaps with a different goal).

The semantics of the top tetrahedron of Fig. 4 are:

- When at $\mathrm{A}$, execute the action $\mathrm{A} \rightarrow \mathrm{B}, \mathrm{X}, \mathrm{C}$.

- When at $\mathrm{B}$, execute either the action $\mathrm{B} \rightarrow \mathrm{X}$ or the action $\mathrm{B} \rightarrow \mathrm{C}$. It does not matter which; pick one, perhaps nondeterministically.

- When at $\mathrm{C}$, execute the action $\mathrm{C} \rightarrow \mathrm{X}$. 
Nondeterminism appears in both the outcomes and choices of the strategy:

1. Nature acts as an adversary during execution of the action $\mathrm{A} \rightarrow \mathrm{B}, \mathrm{X}, \mathrm{C}$, making the outcome uncertain. This can be bad. Fortunately, in the example, the system can compensate, by executing additional actions.

2. The system has available multiple actions at location B. This is good; it provides redundancy, alternate paths. The system can either leave the choice of which action to execute at state B open or it can select a particular action. The tetrahedron in toto leaves the choice open, effectively increasing nondeterminism, perhaps handing the choice over to an adversary. Alternatively, if the system chooses a particular action at state B, it is effectively picking a particular triangular face of the tetrahedron as its true strategy.

Terminology: In the remainder of the paper, we will generally speak of a "strategy" rather than a "plan", in order to avoid the suggestion that strategies are created or discovered by planners. They exist independently of being created or discovered.

\section{Nondeterministic Graphs and Strategy Complexes}

This section makes precise the intuition of the previous section, focusing on nondeterministic graphs; Section 6 introduces stochasticity.

\subsection{Basic Definitions}

\section{Nondeterministic Graphs}

A nondeterministic graph $G=(V, \mathcal{A})$ is a set of states $V$ and a collection of (nondeterministic) actions $\mathcal{A}$. Each $A \in \mathcal{A}$ is a nonempty set of directed edges $\left\{\left(v, u_{1}\right),\left(v, u_{2}\right), \ldots\right\}$, with $v$ and all $u_{i}$ in $V$. We refer to $v$ as $A$ 's source and to each $u_{i}$ as a (nondeterministic) target of $A$. If $A$ has a single target, $A$ is also said to be deterministic.

Action $A$ may be executed whenever the system is at state $v$. When action $A$ is executed, the system moves from state $v$ to one of the targets $u_{i}$. If $A$ has multiple targets, the precise target attained is not known ahead of time. One can imagine an adversary choosing the target.

Distinct actions may have overlapping or identical edge sets. (For instance, two different global motion commands might have the same effect at a given state. We could introduce extra notation to label actions with names, thereby making explicit their individual identity, but the extra notation would be more cumbersome than informative.)

All graphs, sets of states, actions, and collections of actions in this paper are finite.

Remark: Nondeterministic graphs in which each action is deterministic and no two actions have the same edge set are equivalent to standard directed graphs.

Terminology: We speak of a graph "state", reserving the term "vertex" for singleton simplices in simplicial complexes. 


\section{Acyclic Graphs and Subgraphs}

Suppose $G=(V, \mathcal{A})$ is a nondeterministic graph. A possible path of length $k$ in $G$ is a sequence of states $v_{0}, v_{1}, \ldots, v_{k}$ in $V$ such that $\left(v_{i}, v_{i+1}\right) \in A_{i}$, with each $A_{i}$ an action in $\mathcal{A}$, for $i=0, \ldots, k-1$. $G$ is acyclic if none of its possible paths have $v_{0}=v_{k}$ with $k \geq 1$.

A nondeterministic subgraph $H=(W, \mathcal{B})$ of $G$ is a nondeterministic graph in its own right such that $W \subseteq V$ and $\mathcal{B} \subseteq \mathcal{A}$. In particular, any $\mathcal{B} \subseteq \mathcal{A}$ defines a nondeterministic subgraph $H_{\mathcal{B}}=(V, \mathcal{B})$ of $G$. We will say that a collection of actions $\mathcal{B} \subseteq \mathcal{A}$ is acyclic if its induced subgraph $H_{\mathcal{B}}$ is acyclic.

\section{Simplicial Complexes}

An (abstract) simplicial complex $\Sigma$ is a collection of finite sets, such that if $\sigma$ is in $\Sigma$ then so is every subset of $\sigma$ [61]. The elements of $\Sigma$ are called simplices; the elements of a simplex and singleton simplices are both called vertices. Sometimes one refers to the underlying vertex set of a simplicial complex $\Sigma$, which contains all the vertices of $\Sigma$ and may contain vertices that could be present in $\Sigma$ even if they happen not to be. The dimension of a simplex is one less than the number of its elements. If $\tau \subseteq \sigma$, with $\tau, \sigma \in \Sigma$, then one says that $\tau$ is a face of $\sigma$.

We permit the empty simplex $\emptyset$, for combinatorial simplicity $[8,47]$. The complex $\{\emptyset\}$, consisting solely of the empty simplex, is the empty complex. It is also the sphere of dimension -1 . The complex $\emptyset$, consisting of no simplices, is the void complex.

If $\Sigma$ is a simplicial complex, if $\Sigma^{\prime}$ is some subcollection of $\Sigma$, and if $\Sigma^{\prime}$ is a simplicial complex in its own right, then one says that $\Sigma^{\prime}$ is a subcomplex of $\Sigma$.

All simplicial complexes in this paper are finite. Any nonvoid finite simplicial complex has a geometric realization in some Euclidean space, with relative topology the same as its polytope topology [61]. Thus we may view $\Sigma$ as a topological space.

See $[61,6,39]$ for a further introduction to topology and simplicial complexes. See Section 5 for a summary of topological tools used in this paper.

\section{Strategy Complexes}

Given a nondeterministic graph $G=(V, \mathcal{A})$ with $V \neq \emptyset$, let $\Delta_{G}$ be the simplicial complex whose simplices are the acyclic collections of actions $\mathcal{B} \subseteq \mathcal{A}$. If $V=\emptyset$, let $\Delta_{G}=\emptyset$. We refer to $\Delta_{G}$ as $G$ 's strategy complex and to every simplex in $\Delta_{G}$ as a (nondeterministic) strategy.

Observe that no action of $G$ with a self-loop can appear in any simplex of $\Delta_{G}$.

\subsection{Examples}

In what follows, we abbreviate any action $\left\{\left(v, u_{1}\right), \ldots,\left(v, u_{k}\right)\right\}$ by writing $\mathrm{v} \rightarrow \mathrm{u}_{1}, \ldots, \mathrm{u}_{\mathrm{k}}$.

The graph on the left of Fig. 5 is a standard directed graph. Each edge of the directed graph is a possible "action" the system could perform, moving it from some state of the graph to some other state.

The strategy complex of this graph is shown in the right panel of Fig. 5. The biggest simplex one could possibly expect to see in the strategy complex would be a tetrahedron, consisting of all four actions present in the directed graph. However, two of the actions, namely $1 \rightarrow 2$ and $2 \rightarrow 1$, could give rise to a cycle in the graph, so no simplex of the strategy complex can contain both these actions. The complex is in fact generated by two triangles. 

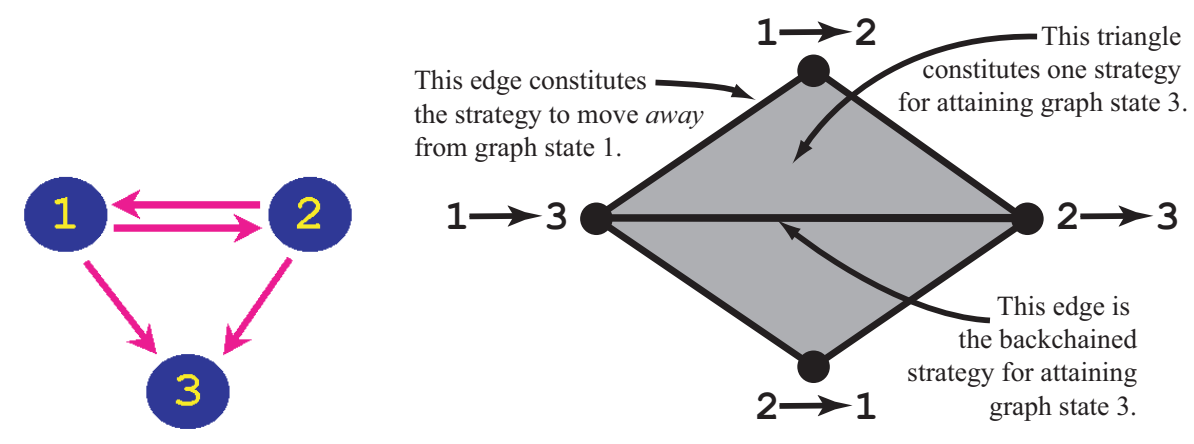

Figure 5: The graph on the left defines the strategy complex shown on the right.

The two triangles, as well as three of the five edges in the complex, constitute strategies for attaining state 3 in the graph. The central edge, consisting of the actions $\{1 \rightarrow 3 ; 2 \rightarrow 3\}$, is the strategy one would obtain by backchaining from state 3 in a traditional fashion.

Observe that a strategy complex may contain strategies for a variety of goals. For instance, the top left edge of the complex in Fig. 5, comprising the set of actions $\{1 \rightarrow 3 ; 1 \rightarrow 2\}$, is a strategy that simply says "move away from state 1."
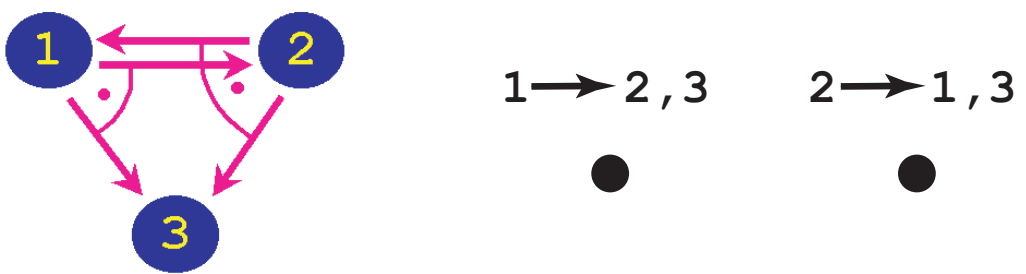

Figure 6: The graph on the left has two nondeterministic actions that could create a cycle, so the strategy complex on the right consists of two isolated vertices.

For contrast, consider the graph of Fig. 6. It contains two actions, one each at states 1 and 2. Each action has two nondeterministic outcomes. The two actions cannot appear together as a simplex since, depending on the actual nondeterministic transitions at runtime, these actions could cause cycling in the graph between states 1 and 2. As a result, the strategy complex consists of two isolated vertices, representing the two strategies "move away from state 1" and "move away from state 2." In particular, there are no strategies guaranteed to attain state 3 from the other two states. 

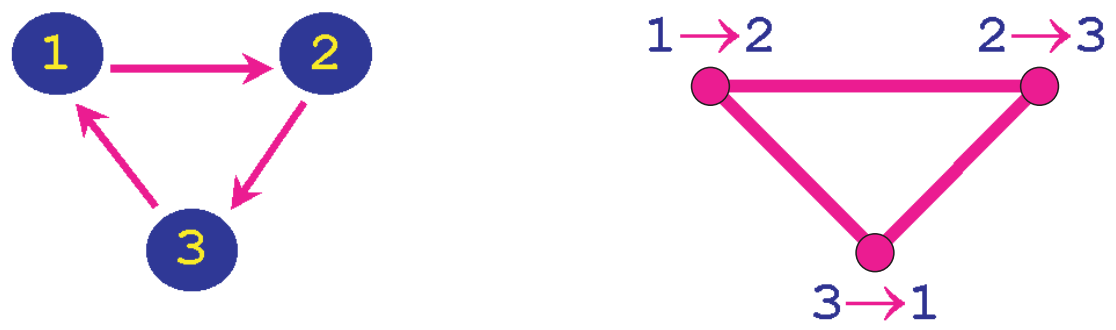

Figure 7: A strongly connected directed graph and its strategy complex. Compare with Fig. 8.
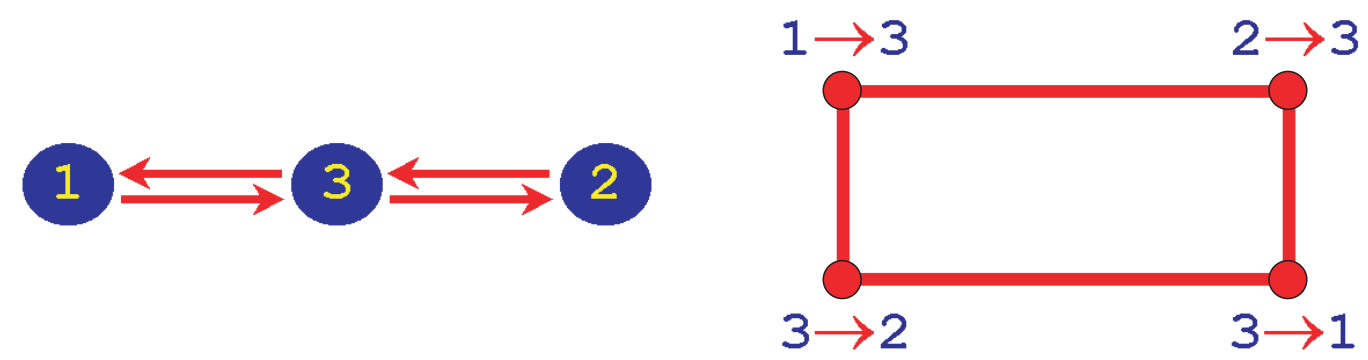

Figure 8: Another strongly connected directed graph and its strategy complex. See also Fig. 7.

It is also instructive to compare the two graphs and strategy complexes shown in Figures 7 and 8. Both graphs are strongly connected directed graphs with three states. The graphs are not isomorphic or even homomorphic, but their strategy complexes are both topological circles.

Indeed, Hultman [44] proved: Any directed graph that can be written as the disjoint union of its strongly connected components generates a strategy complex (he used a different name) topologically similar to a sphere of dimension $n-k-1$, where $n$ is the number of states in the graph and $k$ is the number of strongly connected components. All other directed graphs produce contractible strategy complexes (roughly meaning: they can be shrunk to a point; see Section 5 for a precise definition).

This is a first hint that topology is capturing some significant graph property independent of the detailed structure of a graph. In the case of directed graphs, the strategy complexes always look either like spheres or contractible spaces (homotopically; see Section 5).

Remark: The nondeterministic setting is considerably richer than the deterministic setting of directed graphs. As we will see in Section 13, nondeterministic graphs are able to generate a much larger collection of topological spaces via their strategy complexes, namely all spaces describable by finite simplicial complexes. This means nondeterministic graphs and finite simplicial complexes are essentially identical topological objects. Consequently, one should use topology to study strategies for solving tasks in uncertain discrete spaces. 


\section{Loopback Graphs and Complexes}

Now let us modify the graph of Fig. 5. We will add artificial deterministic transitions from state 3 to each of states 1 and 2. We call these added transitions loopback actions. Think of these loopback actions as "topological electrodes" that will allow us to measure whether the graph contains a guaranteed strategy for attaining state 3 from all states.
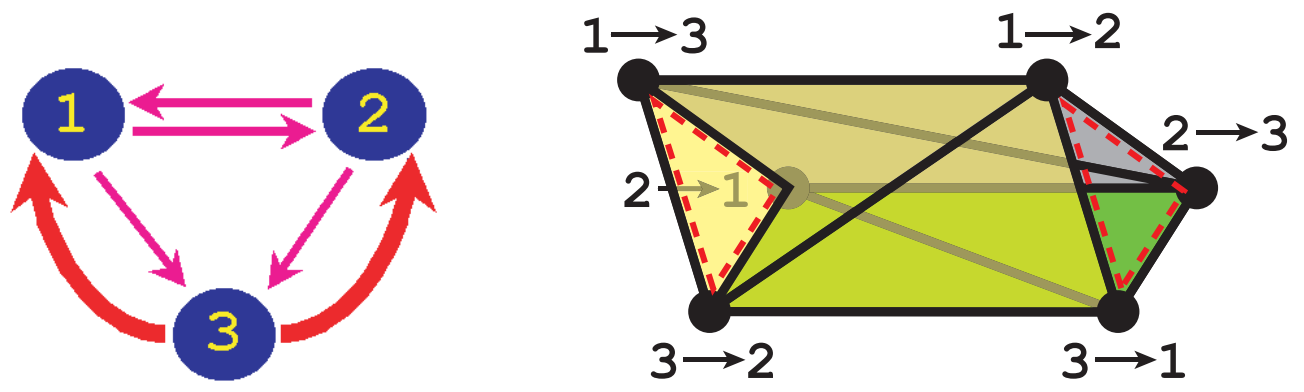

Figure 9: A loopback graph and loopback complex associated with the graph of Fig. 5. The complex contains 6 vertices, 12 edges, and 6 triangles (shaded). The two triangular endcaps outlined in dashed red are not part of the complex, since each gives rise to a cycle in the graph. The complex is homotopic to $S^{1}$, the circle.

The left panel of Fig. 9 shows the resulting loopback graph. Now imagine constructing the strategy complex associated with that graph, as shown in the right panel of the figure. We refer to it as a loopback complex of our original graph. The complex in this case looks roughly like a polygonal cylinder. The complex is homotopic to a circle, as represented by either open end of the cylinder.

"Homotopic" means, in this case, that the complex, viewed as a topological space, can be continuously deformed within itself into a subspace that is topologically a circle. Notice that one cannot continuously deform the complex (within itself) into a point. This is crucial. Homotopy type is an equivalence relation on topological spaces. Circles and points lie in different homotopy equivalence classes. (See Section 5 for precise definitions.)
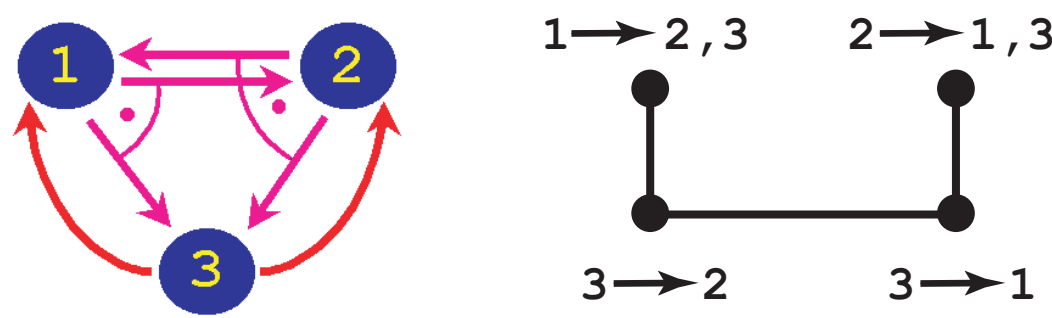

Figure 10: A loopback graph and loopback complex associated with the graph of Fig. 6. The complex is contractible.

In contrast, suppose we add both possible loopback actions at state 3 to the graph of Fig. 6 . The resulting graph and loopback complex are shown in Fig. 10. Now the loopback complex is homotopic to a point; one can continuously deform it within itself to a point. 
The Punch Line: No matter how complicated the nondeterministic graph, if we add all loopback actions to it that transition from some state $s$ to the remaining states, then the resulting loopback complex will always be homotopic either to a sphere or to a point. A sphere tells us that there is a strategy guaranteed to attain state $\mathrm{s}$ from all states in the graph; a point tells us that no such strategy exists. We are beginning to see a topological language by which to characterize system capabilities.

The following definitions and theorem make the previous observation precise. We provide a proof of the theorem following its statement, both to preserve continuity and to build intuition. The reader may nonetheless first wish to review the topological tools discussed in Section 5. The foundations of our proof appear in $[8,44]$. Subsequently, we will generalize these techniques further, namely to stochastic graphs.

Definitions Let $G=(V, \mathcal{A})$ be a nondeterministic graph and suppose $s \in V$ is some desired stop state. We make the following definitions:

- $G$ contains a complete guaranteed strategy for attaining $s$ if there is some acyclic set of actions $\mathcal{B} \subseteq \mathcal{A}$ such that $\mathcal{B}$ contains at least one action with source $v$ for every $v \in V \backslash\{s\}$. Observe that $\mathcal{B}$ cannot contain any actions with source $s$. Moreover, any possible path in the graph $(V, \mathcal{B})$, that terminates at some $v_{k} \neq s$, may be extended to a longer possible path. Iterating, this process converges at $s$, since $\mathcal{B}$ is acyclic.

We say: $\mathcal{B}$ is a complete guaranteed strategy for attaining $s$.

- Define $G_{\leftarrow s}$ to be the nondeterministic graph identical to $G$ except that all actions with source $s$ have been discarded, replaced instead by $(|V|-1)$-many loopback actions $\{(s, v)\}$, each consisting of a single edge from $s$ to some $v$, with $v$ ranging over $V \backslash\{s\}$.

$G_{\leftarrow s}$ is a loopback graph of $G$.

- Define $\Delta_{G_{\leftarrow s}}$ to be the strategy complex associated with $G_{\leftarrow s}$. $\Delta_{G_{\leftarrow s}}$ is a loopback complex of $G$.

- $[n]$ is (standard) shorthand for the set $\{1, \ldots, n\}$.

Theorem 1 (Goal Attainability) Let $G=(V, \mathcal{A})$ be a nondeterministic graph and $s \in V$. If $G$ contains a complete guaranteed strategy for attaining $s$, then $\Delta_{G \leftarrow s}$ is homotopic to the sphere $S^{n-2}$, with $n=|V|$. Otherwise, $\Delta_{G_{\leftarrow s}}$ is contractible.

Proof. We may assume $V=[n]$ and $s=n$. The theorem is trivially true for $n=1$, so suppose $n>1$.

I. Let $\mathcal{B}$ be a complete guaranteed strategy for attaining $s$ and let $\mathcal{A}^{\prime}$ be the actions of $G_{\leftarrow s}$. For each $A \in \mathcal{A}^{\prime}$, define the open polyhedral cone $U_{A}=\bigcap_{(i, j) \in A}\left\{\mathbf{x} \in \mathbf{R}^{n} \mid x_{i}>x_{j}\right\}$. Observe that a set of actions $\left\{A_{1}, \ldots, A_{k}\right\}$ is acyclic if and only if $U_{A_{1}} \cap \cdots \cap U_{A_{k}}$ is not empty. When nonempty, the intersection is contractible. By the Nerve Lemma (see Section 5.3), $\Delta_{G_{\leftarrow s}}$ therefore has the homotopy type of $\bigcup_{A \in \mathcal{A}^{\prime}} U_{A}$. We claim that this union covers all of $\mathbf{R}^{n}$ except for the line on which all coordinates are equal. Thus it is homotopic to $S^{n-2}$.

To see coverage: Clearly no point with all coordinates equal can be in the union. The cones determined by the loopback actions cover all points $\mathbf{x} \in \mathbf{R}^{n}$ for which $x_{n}>x_{i}$, some $i$. 
Suppose some $\mathbf{x}$ in $\mathbf{R}^{n} \backslash\left\{x_{1}=\cdots=x_{n}\right\}$ is not inside any $U_{A}$. Then $x_{i} \geq x_{n}$ for all $i$, with at least one $x_{i}>x_{n}$. Some action $B \in \mathcal{B}$ has that $i$ as a source. $\mathcal{B} \subseteq \mathcal{A}^{\prime}$, so $\mathbf{x} \notin U_{B}$, meaning there is some target $j$ of $B$ such that $x_{j} \geq x_{i}>x_{n}$. Repeating this argument with $j$, etc., produces an arbitrarily long and thus cyclic possible path in $H_{\mathcal{B}}$. Contradiction.

II. If $G$ does not contain a complete guaranteed strategy for attaining $s$, then no simplex of $\Delta_{G \leftarrow s}$ contains actions at all states of $V \backslash\{s\}$. For every simplex $\sigma \in \Delta_{G_{\leftarrow s}}$ there is therefore a unique nonempty maximal set $\tau_{\sigma}$ of loopback actions such that $\sigma \bigcup \tau_{\sigma} \in \Delta_{G \leftarrow s}$. A standard collapsing argument now shows that $\Delta_{G_{\leftarrow s}}$ is contractible (see Section 5.2).

\section{Topological Tools and Homotopy Equivalence}

One of the most important functions of topology is to recognize equivalences between seemingly different objects. This section reviews some of the key topological equivalences and tools for establishing those equivalences. As suggested by Theorem 1, we will use these tools to characterize system capabilities by assigning topological structures to nondeterministic (and stochastic) graphs.

Two simplicial complexes, $\Gamma$ and $\Sigma$, are said to be isomorphic, written $\Gamma \cong \Sigma$, if there is a bijective correspondence between the vertices of the two complexes given by some function $f$, such that $\left\{v_{1}, \ldots, v_{k}\right\}$ is a simplex of $\Gamma$ if and only if $\left\{f\left(v_{1}\right), \ldots, f\left(v_{k}\right)\right\}$ is a simplex of $\Sigma$. In particular, $f$ preserves simplex dimension.

Two topological spaces, $X$ and $Y$, are said to be homeomorphic, written $X \approx Y$, if there exist two continuous functions, $f: X \rightarrow Y$ and $g: Y \rightarrow X$, such that $g \circ f=\mathrm{id}_{X}$ and $f \circ g=\operatorname{id}_{Y}$, where $\operatorname{id}_{X}$ is the identity function on $X$ and $\operatorname{id}_{Y}$ is the identity function on $Y$.

Isomorphic simplicial complexes are homeomorphic when viewed as topological spaces.

$\approx$ is an equivalence relation in the category of topological spaces. It is perhaps the most familiar to the reader. Two homeomorphic spaces really are very much the same intuitively, differing "only" by some continuous transformation with a continuous inverse.

There is a weaker equivalence relation for topological spaces, based on homotopy. This relation is more akin to the notion of "morphing" similarity one sees in computer graphics. The equivalence classes one obtains under homotopy turn out to be useful descriptors for planning in the presence of uncertainty. We can think of these equivalence classes as constituting major nouns of the language we desired in Section 1. We give the definitions next and then some tools for establishing homotopy equivalence.

Suppose $f_{0}: X \rightarrow Y$ and $f_{1}: X \rightarrow Y$ are two continuous functions between topological spaces $X$ and $Y$. One says that $f_{0}$ is homotopic to $f_{1}$, written $f_{0} \simeq f_{1}$, if there exists a continuous function $F: X \times[0,1] \rightarrow Y$ such that $F(x, 0)=f_{0}(x)$ and $F(x, 1)=f_{1}(x)$ for all $x \in X$. (Here $[0,1]$ is the unit interval of the real line.) One can think of $F(\cdot, t)$ as a continuous "morphing" of $f_{0}$ into $f_{1}$ as $t$ varies from 0 to 1 .

Two topological spaces, $X$ and $Y$, are said to be homotopy equivalent (or to have the same homotopy type or to be homotopic), written $X \simeq Y$, if there exist two continuous functions, $f: X \rightarrow Y$ and $g: Y \rightarrow X$, such that $g \circ f \simeq \operatorname{id}_{X}$ and $f \circ g \simeq \operatorname{id}_{Y}$. Observe that the difference between "homeomorphic" and "homotopic" is the difference between compositions that are exactly equal to the identity versus merely homotopic to the identity. Thus homeomorphic spaces are also homotopic. 
A topological space homotopic to a single point is said to be contractible.

It is often difficult to establish homotopy equivalence based on these definitions alone, but there are several key topological tools that one may use instead. We discuss these next. Once again, we also refer the reader to $[61,39,6,8]$ for more extensive treatments.

\subsection{Deformation Retractions}

Suppose $A$ is a subspace of a topological space $X$. A (strong) deformation retraction of $X$ onto $A$ is a continuous function $F: X \times[0,1] \rightarrow X$ such that:

(a) $F(x, 0)=x$ for all $x \in X$,

(b) $F(x, 1) \in A$ for all $x \in X$, and

(c) $F(a, t)=a$ for all $a \in A$ and all $t \in[0,1]$.

$F$ establishes that $X \simeq A[61]$ and one says that $A$ is a deformation retract of $X$. More generally, it is a fact that two spaces are homotopy equivalent precisely when each may be viewed as a deformation retract of some common encompassing space [39].

We encourage the reader to verify the following facts, as warmup for ideas to come:

(1) Let $X$ be all of $n$-dimensional Euclidean space except for the origin. Then $X$ is homotopy equivalent to $S^{n-1}$, the sphere of dimension $n-1$.

(2) Let $X=\mathbf{R}^{n} \backslash\left\{\mathbf{x} \in \mathbf{R}^{n} \mid x_{1}=\cdots=x_{n}\right\}$, that is, $n$-dimensional Euclidean space with a line removed. Then $X$ is homotopy equivalent to $S^{n-2}$. (The proof of Theorem 1 uses this fact.)

\subsection{Collapsibility and Contractibility}

Suppose $\Sigma$ is a simplicial complex and suppose $\tau$ and $\sigma$ are simplices in $\Sigma$ such that $\tau$ is a proper face of $\sigma$. If $\tau$ is a proper face of no other simplex in $\Sigma$, then one can remove both $\tau$ and $\sigma$ from $\Sigma$ to obtain a new complex $\Sigma^{\prime}=\Sigma \backslash\{\tau, \sigma\}$ that has the same homotopy type as $\Sigma$. The process of constructing $\Sigma^{\prime}$ from $\Sigma$ is called an elementary collapse. The reverse process is called an elementary anti-collapse. [6]

It is a fact that a finite simplicial complex is contractible if and only if there is a sequence of elementary collapses and elementary anti-collapses that transforms the complex into a single point. Finding such a sequence is an uncomputable problem (this goes back to a deep result that the word problem for groups is undecidable [63]). The special case in which only elementary collapses are needed is computable; one can try all possibilities. Such complexes are called collapsible. A classic example of a space that is contractible but not collapsible is the "House with Two Rooms" [39].

A finite simplicial complex $\Sigma$ is a cone if there is some vertex $v$ of $\Sigma$ such that $\sigma \bigcup\{v\} \in \Sigma$ whenever $\sigma \in \Sigma$. In this case, $v$ is an apex of the cone. A cone is a classic example of a collapsible complex. 
The following very useful fact appears as Lemma 7.6 in [8] (the collapsibility argument in the proof of Theorem 1 uses this lemma):

Notation: $\quad \sigma \pm v$ means $\sigma \backslash\{v\}$ if $v \in \sigma$ and $\sigma \bigcup\{v\}$ if $v \notin \sigma$.

Lemma 2 (Björner and Welker, [8]) Suppose $\Sigma^{\prime}$ is a subcomplex of a finite simplicial complex $\Sigma$ and suppose for some vertex $v$ of $\Sigma$, the collection $\Sigma \backslash \Sigma^{\prime}$ is closed under the map $\sigma \mapsto \sigma \pm v$. Then $\Sigma$ collapses to $\Sigma^{\prime}$ (via a sequence of elementary collapses) and thus $\Sigma \simeq \Sigma^{\prime}$.

\subsection{The Nerve Lemma}

Suppose $\mathcal{U}$ is some collection of sets (not necessarily distinct). One may define a simplicial complex called the nerve of $\mathcal{U}$, written $\mathcal{N}(\mathcal{U})$, as follows: The simplices of $\mathcal{N}(\mathcal{U})$ are given by the empty simplex and all nonvoid finite subcollections $\left\{U_{1}, \ldots, U_{k}\right\}$ of $\mathcal{U}$ such that the intersection $U_{1} \cap \cdots \cap U_{k}$ is not empty. See $[61,39]$.

Lemma 3 (Nerve Lemma, [39]) Let $X$ be a paracompact topological space. Suppose $\mathcal{U}$ is a collection of open subsets of $X$ whose union covers $X$, such that the intersection of any nonvoid finite subcollection of sets in $\mathcal{U}$ is contractible whenever it is nonempty. Then $X \simeq \mathcal{N}(\mathcal{U})$.

The reader can look up the term "paracompact". See [50, 20, 39]. It is enough for our purposes to know that any topological subspace of Euclidean space is paracompact.

The Nerve Lemma infers global homotopy type from local contractibility. For instance, in Theorem 1, we associated to every action of a nondeterministic graph an open set. The Nerve Lemma allowed us to infer the overall topology of the graph's strategy complex by the intersection properties of these open sets. In Section 7, we will associate open sets with actions in both nondeterministic and stochastic graphs so as to encode the actions' convergence properties. Again, the Nerve Lemma will allow us to infer the topology of the graphs' strategy complexes.

\subsection{The Quillen Fiber Lemma}

Another very useful tool is the Quillen Fiber Lemma. We will state it for partially ordered sets (known as posets). Every simplicial complex $\Sigma$ defines a poset $\mathcal{F}(\Sigma)$, called the face poset of $\Sigma$. The elements of the face poset $\mathcal{F}(\Sigma)$ are the nonempty simplices of the complex $\Sigma$, partially ordered by set inclusion. One can also construct a simplicial complex $\Sigma(P)$, called the order complex, from any poset $P$. The simplices of $\Sigma(P)$ are given by the finite chains $p_{1}<\cdots<p_{k}$ in $P$.

It is a fact that $\Sigma$ and $\Sigma(\mathcal{F}(\Sigma))$ are homeomorphic. Indeed, $\Sigma(\mathcal{F}(\Sigma))$ is the first barycentric subdivision of $\Sigma$, usually written as $\operatorname{sd}(\Sigma)$. Abstractly, $\operatorname{sd}(\Sigma)$ is a new complex, whose nonempty simplices are given by all sets of the form $\left\{\sigma_{1}, \sigma_{2}, \ldots, \sigma_{k}\right\}$, with each $\sigma_{i}$ a nonempty simplex of $\Sigma$, and with $\sigma_{i}$ a proper face of $\sigma_{i+1}$, for $i=1, \ldots, k-1$ [69]. Geometrically, the first barycentric subdivision is a re-triangulation obtained by adding as vertices the centroids (called barycenters) of all simplices in $\Sigma$, then defining new simplices accordingly [71, 61].

Thus posets and simplicial complexes are essentially identical topological objects. For instance, one may speak of the topology of a poset, implicitly meaning the topology of its order complex. We will not elaborate on this connection further, but refer the reader to $[8,6,75]$. 
Notation: If $Q$ is a poset, then $Q_{\leq q}$ denotes the set $\left\{q^{\prime} \in Q \mid q^{\prime} \leq_{Q} q\right\}$, where $\leq_{Q}$ is the partial order on $Q$ (set inclusion in the case of face posets derived from simplicial complexes).

Lemma 4 (Quillen, [68]) Suppose $f: P \rightarrow Q$ is an order-preserving map between two posets. If $f^{-1}\left(Q_{\leq q}\right)$ is contractible for all $q \in Q$, then $P$ and $Q$ are homotopy equivalent.

\subsection{Homotopy Interpretation}

It is still a research question to determine fully what the homotopy type of a graph's strategy complex really means. Much of the rest of the paper will be devoted to understanding that meaning for some important special cases.
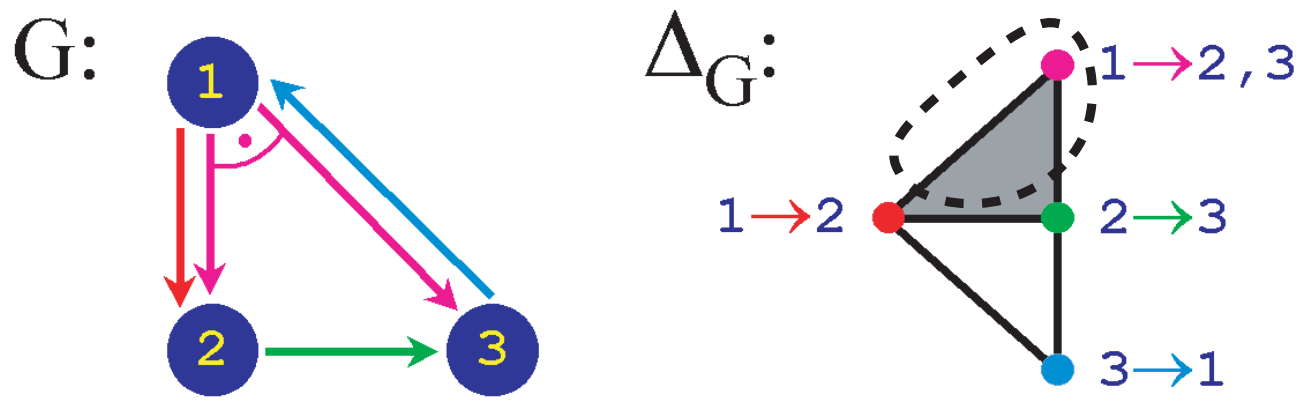

Figure 11: The deterministic action $1 \rightarrow 2$ is more precise than the nondeterministic action $1 \rightarrow 2,3$. The strategy complex makes this explicit, by showing how simplices containing action $1 \rightarrow 2,3$ can collapse away while preserving homotopy type.

There is however something very simple we can observe. Suppose $G=(V, \mathcal{A})$ is a nondeterministic graph. Suppose $G$ contains two distinct actions $A \in \mathcal{A}$ and $B \in \mathcal{A}$ with identical source states such that one action is more precise than the other. Viewed as edge sets, suppose $A \subseteq B$. Intuitively, we would expect never to need action $B$. After all, anything we can be certain of doing with action $B$, we can also be certain of doing with action $A$.

Homotopy equivalence captures this observation via collapsibility. Figure Fig. 11 provides an example.

In particular, suppose $B \in \sigma \in \Delta_{G}$. Let $\tau=\sigma \pm A$. Then $B \in \tau \in \Delta_{G}$ as well. So Lemma 2 tells us that the collection $\Delta^{-}=\left\{\sigma \in \Delta_{G} \mid B \notin \sigma\right\}$ is homotopy equivalent to $\Delta_{G}$. In other words, action $B$ is irrelevant. Observe as well that $\Delta^{-}=\Delta_{G^{-}}$, where $G^{-}$is the nondeterministic graph identical to $G$ except that action $B$ has been removed.

\section{Stochastic Graphs and Strategy Complexes}

\subsection{Stochastic Actions and Graphs}

\section{Stochastic Actions}

Section 3 defined a nondeterministic action to be a nonempty set of directed edges with common source. We now define a stochastic action to be a nonempty set of directed edges with 
common source in which each edge is labeled with a transition probability. Formally, we write a stochastic action $A$ as a nonempty set of labeled pairs in the form $\left\{\left(v, p_{1} u_{1}\right),\left(v, p_{2} u_{2}\right), \ldots\right\}$, with $v$ and all $u_{i}$ elements of some underlying state space $V$. As before, $v$ is $A$ 's source and each $u_{i}$ is a (stochastic) target of $A$. Each label $p_{i}$ is a transition probability.

The semantics of a stochastic action are Markovian: Action $A$ may be executed whenever the system is at state $v$. When action $A$ is executed, the system moves from state $v$ to one of the targets $u_{i}$, selected from all of $A$ 's targets with probability $p_{i}$. We require that each $p_{i}>0$ and that $\sum_{i} p_{i}=1$.

\section{Stochastic Graphs}

A stochastic graph $G=(V, \mathcal{A})$ is a set of states $V$ and a collection of actions $\mathcal{A}$ whose sources and targets all lie in $V . V$ is also known as $G$ 's state space. An action may be either nondeterministic or stochastic. As before, distinct actions may have overlapping or identical edge sets or even identical sets of transition probabilities. And, again, all graphs, sets of states, actions, and collections of actions in this paper are finite.

A stochastic subgraph $H=(W, \mathcal{B})$ of a stochastic graph $G=(V, \mathcal{A})$ is a stochastic graph in its own right such that $W \subseteq V$ and $\mathcal{B} \subseteq \mathcal{A}$.

\section{Remarks:}

- To emphasize: We allow both nondeterministic and stochastic actions in a stochastic graph.

- Determinism: We may view a deterministic action that transitions from state $v$ to state $u$ as a special case of either a nondeterministic action, $\{(v, u)\}$, or a stochastic action, $\{(v, 1 u)\}$. In this paper, it does not matter which.

- Notation: In figures of graphs, we label the edges of a stochastic action with transition probabilities. In figures of strategy complexes, we indicate the vertex corresponding to a stochastic action $A=\left\{\left(v, p_{1} u_{1}\right), \ldots,\left(v, p_{k} u_{k}\right)\right\}$ with the label $\mathrm{v} \rightarrow \mathrm{p}_{1} \mathrm{u}_{1}, \ldots, \mathrm{p}_{\mathrm{k}} \mathrm{u}_{\mathrm{k}}$. We sometimes use this notation in the text as well.

\subsection{Stochastic Acyclicity}
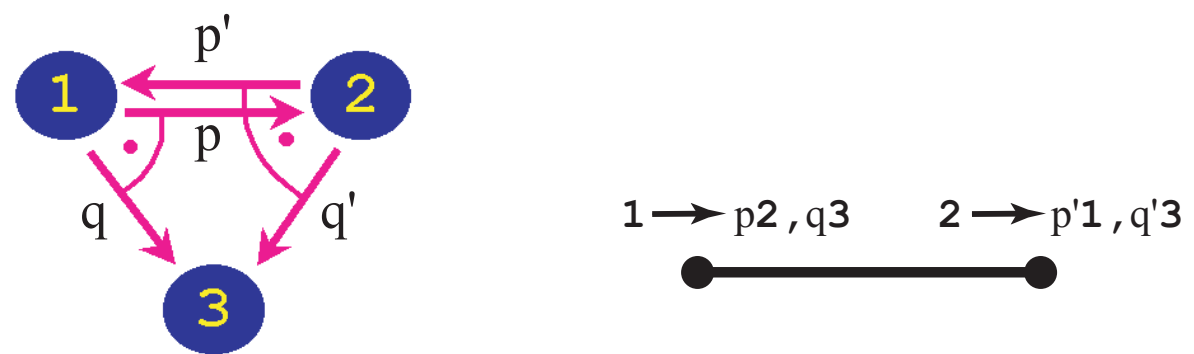

Figure 12: The graph on the left has two stochastic actions that together could create a cycle but must eventually converge to state 3. Consequently, the strategy complex on the right includes not only the individual actions but the full 1-simplex formed by these two actions. 
The two nondeterministic actions of the graph in Fig. 6 did not appear together in a strategy simplex since together they could lead to infinite looping. Now imagine that the actions are merely stochastic, as in Fig. 12. Intuitively, we probably do want to permit both these actions together in a strategy simplex. Although such a strategy might lead to cycling between states 1 and 2, the cycling would be stochastic and therefore not last forever. Eventually the system would converge to state 3 . Indeed, one may even compute the expected convergence times.

We therefore need to modify our definition of acyclicity to account for stochastic convergence. There are two natural approaches, both grounded in the idea that an adversary might try to choose actions and transitions in such a way as to keep the system cycling for as long as possible.

Definition: Given a collection of actions $\mathcal{B}$, let $V_{\mathcal{B}}=\{v \mid v$ is the source of some $B \in \mathcal{B}\}$. We refer to $V_{\mathcal{B}}$ as the start region of $\mathcal{B}$.

Markov Chain Perspective: Suppose $G=(V, \mathcal{A})$ is a stochastic graph (so it may contain either nondeterministic or stochastic actions or both). Let $W$ be some subset of $V_{\mathcal{A}}$. Now imagine an adversary who constructs a Markov chain $M$ as follows:

- For every $v \in W$, the adversary selects some action $A \in \mathcal{A}$ whose source is $v$.

- If the action $A$ is stochastic, that is, $A=\left\{\left(v, p_{1} u_{1}\right), \ldots,\left(v, p_{k} u_{k}\right)\right\}$, then the stochastic transitions of $M$ at $v$ are exactly those given by $A$.

- If the action $A$ is nondeterministic, that is, $A=\left\{\left(v, u_{1}\right), \ldots,\left(v, u_{k}\right)\right\}$, then the adversary further selects one target $u_{i}$ of $A$. There will be exactly one transition of $M$ at $v$, given by an edge from $v$ to $u_{i}$, occurring with probability 1 .

- For every $v \in V \backslash W$, there is a single transition of $M$ at $v$, consisting of a self-loop with probability 1.

We refer to such a construction as a Markov chain $M$ with support $W$ and say $G$ contains $(M, W)$. Observe that in general $G$ may contain many different $(M, W)$.

Convergence Time Perspective: Suppose $G=(V, \mathcal{A})$ is a stochastic graph. Associate to every action $A \in \mathcal{A}$ a nonnegative transition time $\delta_{A}$. Consider the following system of equations:

$t_{v}=\max \left(\max _{\substack{A \in \mathcal{A} \\ A=\left\{\left(v, u_{j}\right)\right\}}}\left(\max _{j} t_{u_{j}}+\delta_{A}\right), \max _{\substack{A \in \mathcal{A} \\ A=\left\{\left(v, p_{j} u_{j}\right)\right\}}}\left(\sum_{j} p_{j} t_{u_{j}}+\delta_{A}\right)\right)$, if $v \in V_{\mathcal{A}}$

$t_{v}=0, \quad$ if $v \notin V_{\mathcal{A}}$. 
The reader may recognize System (1) as describing a maximization over expected durations of random walks with variable step times [32], induced by Markov chains $\left(M, V_{\mathcal{A}}\right)$ contained in $G$. It is a form of Bellman's equation, representing an optimization from an imagined adversary's perspective, who is trying to maximize the graph's convergence times.

The convergence times are 0 for all states at which there are no actions. Otherwise, the maximizations appearing in System (1) describe adversarial choices. At any state $v$, the adversary maximizes over all actions $A$ with source $v$. If $A$ is a nondeterministic action, the adversary performs an additional maximization over the convergence times of all targets of $A$; if $A$ is stochastic, there appears an expectation over the convergence times of the target states.

Reminder: A recurrent class of a Markov chain $M$ is a set of states $R$ of $M$ such that the probability of reaching any state of $R$ from any other state of $R$ is 1 , while the probability of reaching any state outside $R$ is 0 . The restriction of $M$ to $R$ induced by transitions at states of $R$ therefore itself defines a Markov chain. If $R$ consists of a single state, that state is called absorbing. $[32,48]$

The following lemma establishes an equivalence between the Markov chain perspective and the convergence time perspective:

Lemma 5 (Stochastic Acyclicity) Let $G=(V, \mathcal{A})$ be a stochastic graph with associated nonnegative action transition times $\left\{\delta_{A}\right\}_{A \in \mathcal{A}}$.

System (1) has a unique finite solution if and only if the only recurrent classes of any Markov chain with support $W$ contained in $G$ are formed by the absorbing states $V \backslash W$.

Moreover, when the solution is unique and finite, it is nonnegative, that is, $t_{v} \geq 0$ for all $v \in V$.

Proof. We omit the details of the proof. The basic techniques are similar to those used in Markov Decision Processes. We point to $[32,48,78]$.

The previous lemma permits us to move back and forth between the Markov chain and convergence time perspectives. Often it is best to reason directly about the recurrent classes induced by a stochastic graph but easier to compute convergence times using System (1). In particular, when System (1) has a unique finite solution one can use the system in an iterative fashion to obtain that solution.

\subsection{Stochastic Strategy Complexes}

We now make some further definitions, leading to strategy complexes in the stochastic setting.

\section{Stochastically Acyclic Collections of Actions}

Suppose $G=(V, \mathcal{A})$ is a stochastic graph. $G$ is stochastically acyclic if System (1) has a unique finite solution for some (and thus any) set of nonnegative action transition times $\left\{\delta_{A}\right\}_{A \in \mathcal{A}}$. Similarly, we say that a collection of actions $\mathcal{B} \subseteq \mathcal{A}$ is stochastically acyclic if the induced subgraph $H_{\mathcal{B}}=(V, \mathcal{B})$ is stochastically acyclic. 
When $\mathcal{B}$ is stochastically acyclic, we may view $\mathcal{B}$ as a strategy for moving the system into the complement of $\mathcal{B}$ 's start region $V_{\mathcal{B}}$ : If the initial state of the system lies inside $V_{\mathcal{B}}$, then moving under actions of $\mathcal{B}$, the system will eventually stop at some state inside $V \backslash V_{\mathcal{B}}$. If the system initially starts in $V \backslash V_{\mathcal{B}}$, then it remains there. Given specific nonnegative action transition times $\left\{\delta_{B}\right\}_{B \in \mathcal{B}}$, we refer to the solution of System (1), written out for $H_{\mathcal{B}}$ with those transition times, as the worst-case expected convergence times of $\mathcal{B}$.

\section{Stochastic Strategy Complexes}

Given a stochastic graph $G=(V, \mathcal{A})$ with $V \neq \emptyset$, let $\Delta_{G}$ be the simplicial complex whose simplices are the stochastically acyclic collections of actions $\mathcal{B} \subseteq \mathcal{A}$. If $V=\emptyset$, let $\Delta_{G}=\emptyset$.

This definition is identical to the one we gave earlier for nondeterministic graphs, but our notion of "acyclic collections of actions" has now been enlarged to include stochastic actions. Again, we refer to $\Delta_{G}$ as $G$ 's strategy complex and to every simplex in $\Delta_{G}$ as a (stochastic) strategy.

\section{Time-Bounded Strategy Complexes}

System (1) allows us to define a tower of strategy complexes for any graph $G=(V, \mathcal{A})$.

Suppose we associate nonnegative transition times $\left\{\delta_{A}\right\}_{A \in \mathcal{A}}$ to the actions of $G$. To every $\sigma \in \Delta_{G}$ we can then associate a maximal worst-case expected convergence time, $t_{\text {max }}(\sigma)$, defined to be the maximum time $t_{v}$ obtained as a solution to System (1) when written out for the graph $(V, \sigma)$.

Let $T \geq 0$ be given. Define $\Delta_{G}^{T}=\left\{\sigma \in \Delta_{G} \mid t_{\max }(\sigma) \leq T\right\}$. Then $\Delta_{G}^{T}$ is a subcomplex of $\Delta_{G}$, representing all strategies whose maximal worst-case expected convergence times are no greater than $T$. (Exercise for the reader: Prove that removing actions from a simplex $\sigma$ cannot raise $t_{\max }(\sigma)$, as is required to infer that $\Delta_{G}^{T}$ is a simplicial complex.) 


\section{Covering Sets}

We wish to understand the topology of strategy complexes and thus the capabilities of nondeterministic and stochastic graphs. Let us adopt a technique that appeared in the proof of Theorem 1 and associate to each action an open set. The topology of the resulting collection of open sets will be the topology of a graph's strategy complex.

\subsection{Homogeneous Covering Sets}

Definition: Suppose $G=(V, \mathcal{A})$ is a stochastic graph with $V \neq \emptyset$. We can assume for simplicity that $V=[n]$, with $n \geq 1$. We associate to each $A \in \mathcal{A}$ an homogeneous open subset $U_{A}$ of $\mathbf{R}^{n}$, which we refer to as a covering set:

- If the action $A$ is stochastic, that is, $A=\left\{\left(i, p_{j} j\right)\right\}$ for some set of targets $\{j\}$, then

$$
U_{A}=\left\{\begin{array}{l|l}
\mathbf{x} \in \mathbf{R}^{n} \mid x_{i}>\sum_{j} p_{j} x_{j}
\end{array}\right\} .
$$

- If the action $A$ is nondeterministic, that is, $A=\{(i, j)\}$ for some set of targets $\{j\}$, then

$$
U_{A}=\bigcap_{j}\left\{\mathbf{x} \in \mathbf{R}^{n} \mid x_{i}>x_{j}\right\}
$$

In the stochastic case, the open set $U_{A}$ is a halfspace of $\mathbf{R}^{n}$ whose defining hyperplane has a normal determined by action $A$ 's transition probabilities. In the nondeterministic case, the open set $U_{A}$ is the intersection of several such halfspaces, one for each possible nondeterministic target. Observe that the defining hyperplanes all contain the line $\left\{\mathbf{x} \in \mathbf{R}^{n} \mid x_{1}=\cdots=x_{n}\right\}$.

Remark: The collection of all hyperplanes of the form $\left\{\mathbf{x} \in \mathbf{R}^{n} \mid x_{i}=x_{j}\right\}$ is known classically as the Type $A$ braid arrangement in $\mathbf{R}^{n}$ [75]; it is very useful for studying the poset of all posets on $n$ items [8]. It should therefore come as no surprise that the open sets $\left\{U_{A}\right\}_{A \in \mathcal{A}}$ will allow us to infer the topology of $G$ 's strategy complex. In particular, for a nondeterministic graph $G$, we are simply looking at a subposet of that overall poset of posets on $n$ items. For a stochastic graph $G$, we have effectively created a poset of stochastic partial orders, each of which we may think of as a collection of Markov chains all of whose states are either transient or trivially absorbing (that was the gist of Lemma 5).

\subsection{Affine Covering Sets}

Definition: In order to study the topology of time-bounded strategy complexes, it is useful to define affine covering sets. Suppose we associate nonnegative transition times $\left\{\delta_{A}\right\}_{A \in \mathcal{A}}$ to the actions of $G$. Then we can define for each $A \in \mathcal{A}$ an affine open subset $U_{A}^{+}$of $\mathbf{R}^{n}$ :

- If $A=\left\{\left(i, p_{j} j\right)\right\}$ is stochastic, then $U_{A}^{+}=\left\{\mathbf{x} \in \mathbf{R}^{n} \mid x_{i}>\sum_{j} p_{j} x_{j}+\delta_{A}\right\}$.

- If $A=\{(i, j)\}$ is nondeterministic, then $U_{A}^{+}=\bigcap_{j}\left\{\mathbf{x} \in \mathbf{R}^{n} \mid x_{i}>x_{j}+\delta_{A}\right\}$. 


\subsection{Inferring Topology from Covering Sets}

The following lemma shows that $\Delta_{G}$ and $\mathcal{N}\left(\left\{U_{A}\right\}_{A \in \mathcal{A}}\right)$ are isomorphic simplicial complexes (and therefore homeomorphic and homotopic). The lemma generalizes to stochastic graphs a statement that appeared for nondeterministic graphs early in the proof of Theorem 1.

Lemma 6 (Homogeneous Nerve) Let $G=(V, \mathcal{A})$ be a stochastic graph with $V=[n]$, $n>0$. Suppose $\emptyset \neq \mathcal{B} \subseteq \mathcal{A}$. Then:

$$
\bigcap_{B \in \mathcal{B}} U_{B} \neq \emptyset \quad \text { if and only if } \quad \mathcal{B} \text { is stochastically acyclic. }
$$

Proof. Recall that $H_{\mathcal{B}}$ means the subgraph $(V, \mathcal{B})$ of $G$.

I. Suppose $\bigcap_{B \in \mathcal{B}} U_{B} \neq \emptyset$.

Choose $\mathbf{x}^{*} \in \mathbf{R}^{n}$ so that $\mathbf{x}^{*} \in U_{B}$ for all $B \in \mathcal{B}$. Define $\delta_{B}$ for each $B \in \mathcal{B}$ as follows:

If $B=\left\{\left(i, p_{j} j\right)\right\}$ is stochastic, let $\delta_{B}=x_{i}^{*}-\sum_{j} p_{j} x_{j}^{*}$.

If $B=\{(i, j)\}$ is nondeterministic, let $\delta_{B}=x_{i}^{*}-\max _{j}\left(x_{j}^{*}\right)$.

Observe that each $\delta_{B}>0$.

Now consider the system of equations:

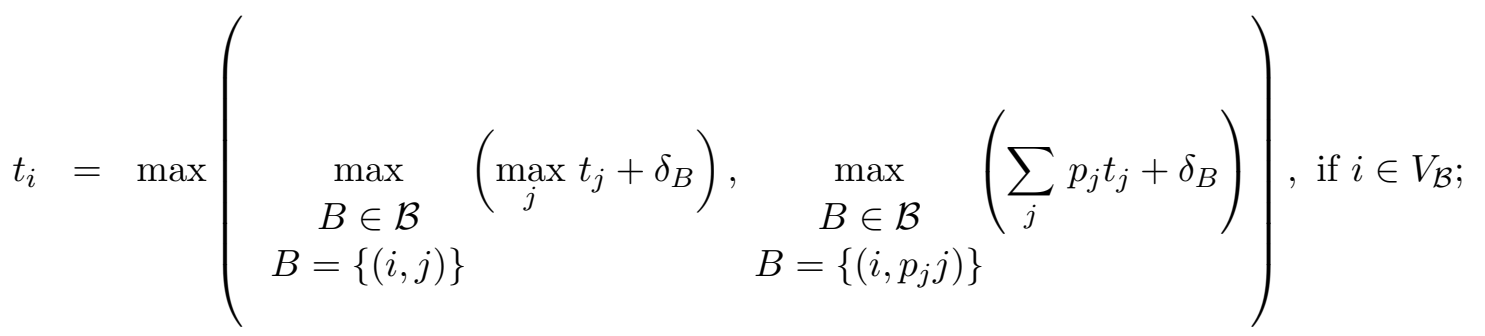

$t_{i}=x_{i}^{*}, \quad$ if $i \notin V_{\mathcal{B}}$.

By construction, System (2) has at least one finite solution, given by $\mathbf{x}^{*}$, that is, $t_{i}=x_{i}^{*}$, for all $i \in V$. (In fact, the solution holds for all actions $B \in \mathcal{B}$, that is, the maximum at state $i$ in System (2) occurs for every action of $\mathcal{B}$ with source $i$.)

Now suppose that $H_{\mathcal{B}}$ contains a Markov chain $(M, W)$ whose support $W$ is a recurrent class. Let $P=\left(p_{i j}\right)$ be the probability transition matrix of $M$ restricted to $W$. $P$ is a stochastic matrix in its own right since $W$ is a recurrent class. The transition probabilities $p_{i j}$ are determined from actions of $\mathcal{B}$ via the process outlined on page 22 . In particular, for every $i \in W$, some action $B \in \mathcal{B}$ gives rise to $M$ 's transitions at state $i$. Define $\delta_{i}=\delta_{B}$.

Combining this construction with System (2), we see that:

$$
x_{i}^{*} \geq \sum_{j \in W} p_{i j} x_{j}^{*}+\delta_{i}, \quad \text { for all } i \in W .
$$


That is only possible if $\delta_{i} \leq 0$ for some $i \in W$, since $P$ is a stochastic matrix. Contradiction. So, by Lemma $5, \mathcal{B}$ must be stochastically acyclic.

II. Suppose $\mathcal{B}$ is stochastically acyclic.

For each $B \in \mathcal{B}$, let $\delta_{B}>0$ be arbitrary. Now write out System (1) for $H_{\mathcal{B}}$ :

$$
\begin{aligned}
& t_{i}=\max \left(\begin{array}{l}
\left.\max _{B \in \mathcal{B}}\left(\max _{j} t_{j}+\delta_{B}\right), \max _{B \in \mathcal{B}}\left(\sum_{j} p_{j} t_{j}+\delta_{B}\right)\right) \\
B=\{(i, j)\}
\end{array}\right) \text {, if } i \in V_{\mathcal{B}} ; \\
& t_{i}=0, \quad \text { if } i \notin V_{\mathcal{B}} .
\end{aligned}
$$

By assumption, this system has a unique finite solution, call it $\mathbf{t}^{*}$.

Now consider $B \in \mathcal{B}$ :

If $B=\left\{\left(i, p_{j} j\right)\right\}$ is stochastic, then $t_{i}^{*} \geq \sum_{j} p_{j} t_{j}^{*}+\delta_{B}>\sum_{j} p_{j} t_{j}^{*}$.

If $B=\{(i, j)\}$ is nondeterministic, then $t_{i}^{*} \geq \max _{j}\left(t_{j}^{*}\right)+\delta_{B}>\max _{j}\left(t_{j}^{*}\right)$.

So $\mathbf{t}^{*} \in U_{B}$ for all $B \in \mathcal{B}$, establishing that $\bigcap_{B \in \mathcal{B}} U_{B} \neq \emptyset$.

In order to obtain the topology of time-bounded strategy complexes we need to be a little more careful. There may exist gaps in the affine covering sets near the line on which all coordinates are equal. We will therefore intersect the covering sets with the boundary of a polyhedral cylinder designed to measure convergence times.

Definition: Given real $r>0$, let $C_{r}=\left\{\mathbf{x} \in \mathbf{R}^{n}|| x_{i}-x_{j} \mid<r\right.$ for all $\left.i, j \in[n]\right\}$ and let $\partial C_{r}$ be the boundary of $C_{r}$.

Observe: $\partial C_{r}$ is a polyhedral cylinder with axis given by the line $\left\{\mathbf{x} \in \mathbf{R}^{n} \mid x_{1}=\cdots=x_{n}\right\}$ and with $r$ the cylinder's "radius".

Lemma 7 (Affine Nerve) Let $G=(V, \mathcal{A})$ be a stochastic graph with associated nonnegative action transition times $\left\{\delta_{A}\right\}_{A \in \mathcal{A}}$. Assume $V=[n]$ and $n>0$. Let $T \geq 0$ be given.

There exists $\epsilon_{T}>0$, such that for every $0<\epsilon<\epsilon_{T}$ :

For every $\emptyset \neq \mathcal{B} \subseteq \mathcal{A}, \mathcal{B}$ is stochastically acyclic with $t_{\max }(\mathcal{B}) \leq T$ if and only if

$$
\bigcap_{B \in \mathcal{B}} U_{B}^{+} \bigcap \partial C_{T+\epsilon} \neq \emptyset
$$

We omit the proof, except to note that the existence of $\epsilon_{T}$ in the proof depends on finiteness of $G$. We now move directly to the key theorem that describes the topology of strategy complexes in terms of the topology of covering sets in $\mathbf{R}^{n}$ : 
Theorem 8 (Cover Homotopy) Let $G=(V, \mathcal{A})$ be a stochastic graph with $V=[n], n>0$.

$$
\text { Then } \Delta_{G} \simeq \bigcup_{A \in \mathcal{A}} U_{A} \text {. }
$$

Let the action transition times of $G$ be given by nonnegative numbers $\left\{\delta_{A}\right\}_{A \in \mathcal{A}}$ and let $T \geq 0$.

$$
\text { Then } \Delta_{G}^{T} \simeq \bigcup_{A \in \mathcal{A}} U_{A}^{+} \bigcap \partial C_{T+\epsilon},
$$

with $0<\epsilon<\epsilon_{T}$ and $\epsilon_{T}$ given as per Lemma $\%$.

Proof. First, observe that whenever a set of the form $U_{A_{1}} \cap \cdots \cap U_{A_{k}}$ is nonempty, then it is convex hence contractible. We therefore obtain

$$
\Delta_{G} \cong \mathcal{N}\left(\left\{U_{A}\right\}_{A \in \mathcal{A}}\right) \simeq \bigcup_{A \in \mathcal{A}} U_{A}
$$

The isomorphism $\cong$ follows from Lemma 6 and the homotopy equivalence $\simeq$ follows from the Nerve Lemma.

Second, observe that whenever a set of the form $U_{A_{1}} \cap \cdots \cap U_{A_{k}} \cap \partial C_{T+\epsilon}$ is nonempty, then, while it may not be convex, it is the deformation retract of a convex set, so is contractible. Consequently, we may apply Lemma 7 and the Nerve Lemma to conclude

$$
\Delta_{G}^{T} \cong \mathcal{N}\left(\left\{U_{A}^{+} \bigcap \partial C_{T+\epsilon}\right\}_{A \in \mathcal{A}}\right) \simeq \bigcup_{A \in \mathcal{A}} U_{A}^{+} \bigcap \partial C_{T+\epsilon}
$$
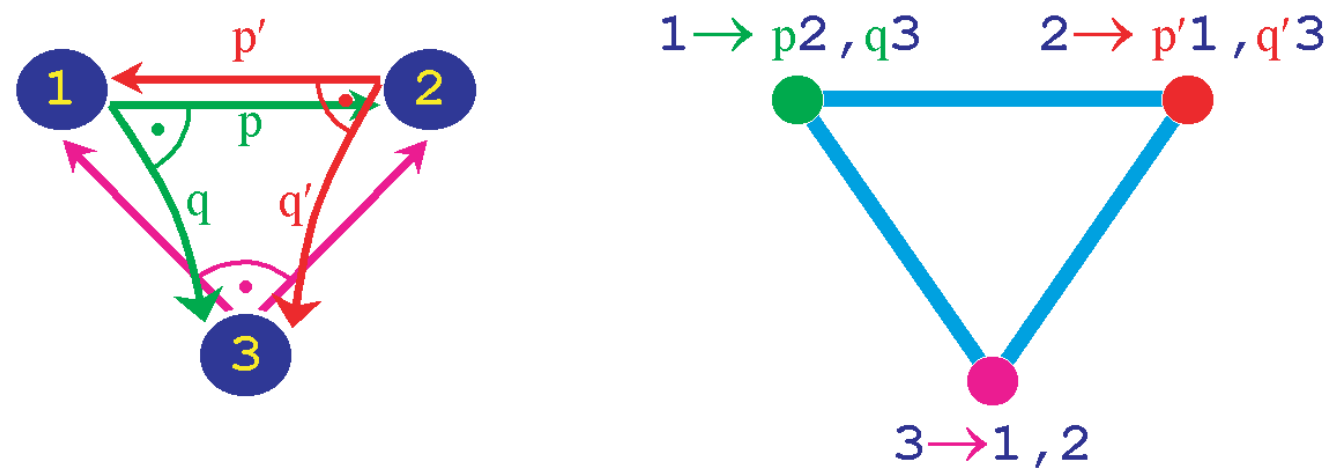

Figure 13: The graph on the left has two stochastic actions (at states 1 and 2) and one nondeterministic action (at state 3 ). Its strategy complex, shown on the right, is the boundary of a triangle.

As an example, let $G$ be the graph of Fig. 13. The graph has three states and three actions, one at each state. Two of the actions are stochastic and one is nondeterministic. $\Delta_{G}$ is the boundary complex of a triangle.

Fig. 14 shows the previous lemmas and theorem in action, depicting the covering sets $\left\{U_{1}^{+}, U_{2}^{+}, U_{3}^{+}\right\}$associated with the three actions of $G$, assuming all action transition times 


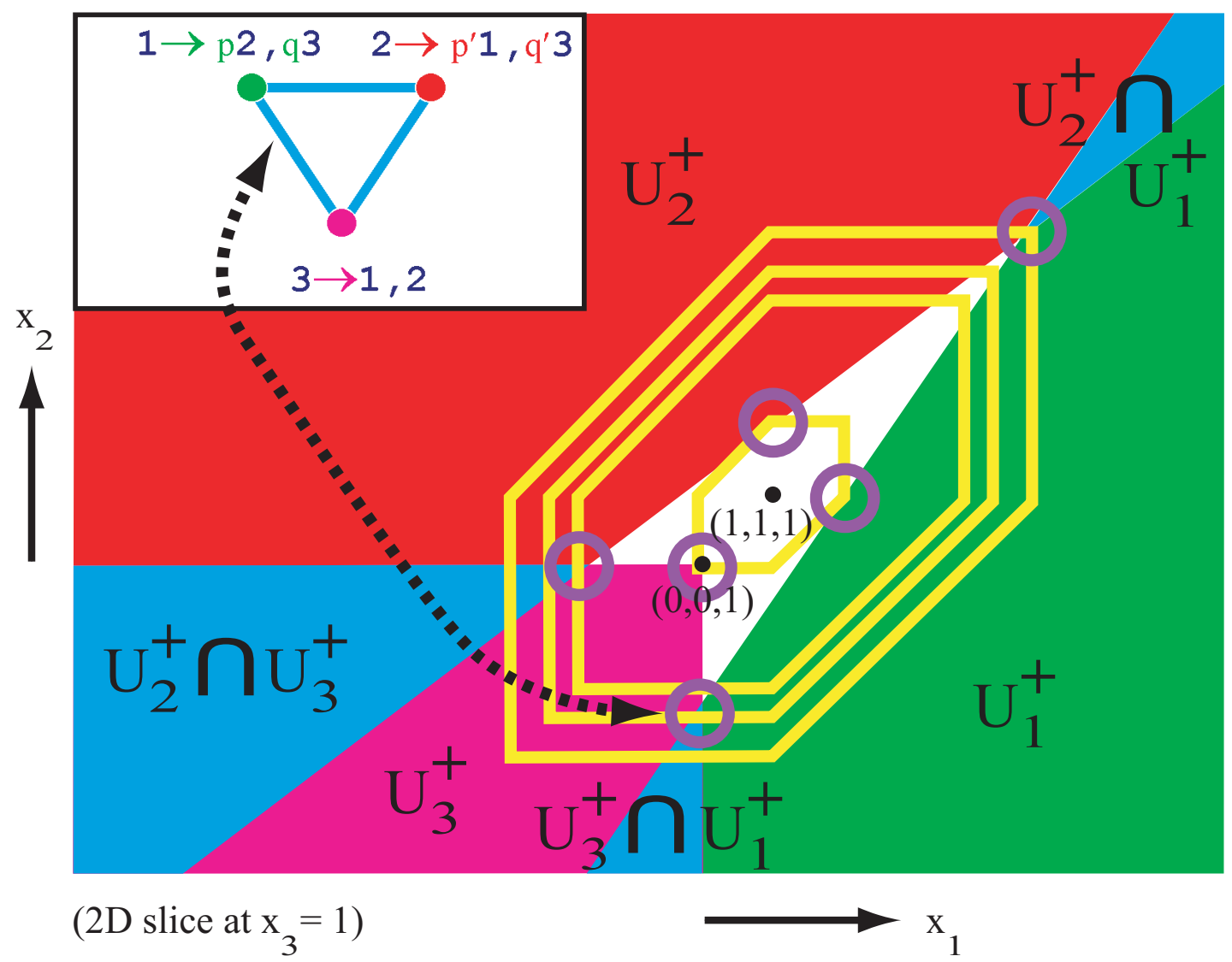

Figure 14: This figure shows a two-dimensional slice of the covering sets associated with the actions of the graph in Fig. 13, along with intersections of these covering sets. The slice describes the $\left(x_{1}, x_{2}\right)$-shape of the covering sets at $x_{3}=1$. The figure also shows $\partial C_{r}$ (drawn in yellow) for various critical radii $r$ (roughly), with the critical events circled. For reference, the graph's strategy complex appears in the upper left corner. 
are 1 and assuming $p^{\prime}>p>\frac{2}{3}$. The figure actually shows the $\left(x_{1}, x_{2}\right)$-slice of the cover $\left\{U_{1}^{+}, U_{2}^{+}, U_{3}^{+}\right\}$at $x_{3}=1$. Since the covering sets are invariant with respect to a translation along the direction $(1,1,1)$, it is enough to look at such two-dimensional slices to determine the topologies of $\Delta_{G}$ and $\Delta_{G}^{T}$.

The covering set $U_{3}^{+}$associated with the action $3 \rightarrow 1,2$ is the intersection of two halfspaces in $\mathbf{R}^{3}$. It therefore appears as the quadrant $\left\{\left(x_{1}, x_{2}\right) \mid x_{1}<0\right.$ and $\left.x_{2}<0\right\}$ in Fig. 14. $U_{1}^{+}$and $U_{2}^{+}$each arise from stochastic actions and thus appear as affine halfspaces in Fig. 14.

The boundary of the cylinder $C_{r}$ is drawn for several critical radii, namely those at which some simplex of $\Delta_{G}$ appears. The innermost cylinder has radius $r=1+\epsilon$. Since each action has transition time 1 , this cylinder just touches each of the covering sets $U_{1}^{+}, U_{2}^{+}$, and $U_{3}^{+}$. From a nerve perspective, this geometry produces the three vertices of the complex $\Delta_{G}$. As the radius grows, the other simplices of $\Delta_{G}$ appear. The correspondence between the covering geometry and the complex topology is highlighted with a dashed double arrow for one critical event: The intersection $U_{1}^{+} \cap U_{3}^{+}$in the cover $\left\{U_{1}^{+}, U_{2}^{+}, U_{3}^{+}\right\}$corresponds to the 1-simplex $\left\{1 \rightarrow \mathrm{p} 2, \mathrm{q}^{3} ; 3 \rightarrow 1,2\right\}$ in the complex $\Delta_{G}$.

\section{Controllability of Motions in Stochastic Graphs}

Theorem 1 provides a topological test for guaranteed goal reachability in nondeterministic graphs. (A similar result holds in the stochastic setting.) We seek a more general result, along the lines of Hultman's topological characterization of strong connectivity in directed graphs. We have the tool of covering sets from Section 7, so let us use that tool to extract topology from strategies. First, here are some

Definitions: Throughout, let $G=(V, \mathcal{A})$ be a stochastic graph and let $S$ be a nonempty subset of $V$ ( $S$ stands for "stop states").

- $G$ is a stochastic strategy for attaining $S$ if:

(i) $G$ is stochastically acyclic, and

(ii) $V \backslash V_{\mathcal{A}} \subseteq S \subseteq V$.

This definition ensures that $G$ contains actions at all states outside of $S$ and that motions under those actions eventually converge (in a subset of $S$ ).

Observe that $V_{\mathcal{A}}$ cannot be all of $V$, since $G$ is stochastically acyclic.

- $G$ contains a complete stochastic strategy for attaining $S$ (on the state space $V$ ) if there is some set of actions $\mathcal{B} \subseteq \mathcal{A}$ such that $H_{\mathcal{B}}=(V, \mathcal{B})$ is a stochastic strategy for attaining $S$. (This definition is consistent with the definition of complete guaranteed strategy from Section 4, p.16.)

In this case: $\quad-$ We refer to both $\mathcal{B}$ and $H_{\mathcal{B}}$ as being a complete stochastic strategy for attaining $S$ (in the graph $G$ or on the state space $V$ ).

- We say $S$ is a stochastically attainable goal (in $G$ or within $V$ ). 
- Suppose $\mathcal{I}$ is a subset of $V$ ( $\mathcal{I}$ stands for "initial states"). G contains a stochastic strategy for attaining $S$ from $\mathcal{I}$ if $G$ contains a subgraph $H=(W, \mathcal{B})$ such that $\mathcal{I} \bigcup S \subseteq W$ and $H$ is a stochastic strategy for attaining $S$. We refer to $H$ as a stochastic strategy for attaining $S$ from $\mathcal{I}$. Of course, we may also view $H$ as a complete stochastic strategy for attaining $S$, now on the state space $W$.

This definition captures the idea that a strategy for attaining some set of states from some other set of states may require moving through some intermediate states, but not necessarily all of $V$.

We will presently focus on cases in which $\mathcal{I}$ and $S$ are both singleton sets. In such cases, we speak of attaining one state from another.

\subsection{Connectivity: Covers and Chains}

The following two lemmas capture the idea that stochastically certain connectivity between two states in a stochastic (or nondeterministic) graph implies coverage of a particular halfspace in $\mathbf{R}^{n}$ by the graph's covering sets. This fact appeared as a special case in the proof of Theorem 1. Subsequently, we will use these two lemmas to characterize graph controllability (Theorems 11 and 12).

Lemma 9 (Stochastic Connectivity) Let $G=(V, \mathcal{A})$ be a stochastic graph with $V=[n]$, $n>0$. Let $\ell, k \in V$.

Suppose $G$ contains a stochastic strategy for attaining state $k$ from state $\ell$. Then

$$
\left\{\mathbf{x} \in \mathbf{R}^{n} \mid x_{\ell}>x_{k}\right\} \subseteq \bigcup_{A \in \mathcal{A}} U_{A} .
$$

Proof. While we could give a direct proof, we may also view this lemma as a special case of Lemma 10, with all action transition times zero. That lemma appears next.

Lemma 10 (Time-Bounded Stochastic Connectivity) Let $G=(V, \mathcal{A})$ be a stochastic graph with associated nonnegative action transition times $\left\{\delta_{A}\right\}_{A \in \mathcal{A}}$.

Assume $V=[n]$ and $n>0$. Let $\ell, k \in V$ be given.

Suppose $G$ contains a stochastic strategy for attaining state $k$ from state $\ell$. Let $t_{\ell}$ be the worst-case expected convergence time to attain $k$ from $\ell$, as given by the solution of System (1) when written out for this stochastic strategy. Then

$$
\left\{\mathbf{x} \in \mathbf{R}^{n} \mid x_{\ell}>x_{k}+t_{\ell}\right\} \subseteq \bigcup_{A \in \mathcal{A}} U_{A}^{+} .
$$

Proof. Let $H=(W, \mathcal{B})$ be the subgraph of $G$ constituting the given stochastic strategy for attaining $k$ from $\ell$. So $\ell, k \in W$. We can assume without loss of generality that $W=[k]$, so it is enough to show that

$$
\left\{\mathbf{x} \in \mathbf{R}^{k} \mid x_{\ell}>x_{k}+t_{\ell}\right\} \subseteq \bigcup_{B \in \mathcal{B}} U_{B}^{+}
$$

with each set $U_{B}^{+}$now a subset of $\mathbf{R}^{k}$. 
If $\ell=k$, there is nothing to prove, since the set on the left is the empty set, which is a subset of all sets. So assume $\ell \neq k$.

Here is System (1) written out for $H$ :

$$
t_{i}=\max \left(\begin{array}{cc}
\max _{B \in \mathcal{B}}\left(\max _{j} t_{j}+\delta_{B}\right), & \max _{\substack{B \in \mathcal{B} \\
B=\{(i, j)\}}}\left(\sum_{j} p_{j} t_{j}+\delta_{B}\right) \\
B=\left\{\left(i, p_{j} j\right)\right\}
\end{array}\right), \text { for } 1 \leq i<k ;
$$

$t_{k}=0$.

Since $H$ is stochastically acyclic, this system has a unique finite solution with all $t_{i} \geq 0$.

Suppose there is some $\mathbf{x}^{*} \in \mathbf{R}^{k}$ such that $x_{\ell}^{*}>x_{k}^{*}+t_{\ell}$ but $\mathbf{x}^{*} \notin \bigcup_{B \in \mathcal{B}} U_{B}^{+}$. Since the sets $U_{B}^{+}$are invariant with respect to translation along the line $\left\{\mathbf{x} \in \mathbf{R}^{k} \mid x_{1}=\cdots=x_{k}\right\}$, we can assume without loss of generality that $x_{k}^{*}=0$.

Now define a Markov chain $M$ contained in $H$, with support $[k-1]$ :

- At state $k$, let $M$ remain at $k$ with probability 1 .

- At state $i \in[k-1]$, pick some action $B \in \mathcal{B}$ whose source is $i$. Such an action must exist, since $H$ is a complete stochastic strategy for attaining $k$ on the state space $W$. Let $\delta_{i}=\delta_{B}$

- If $B$ is stochastic, that is, $B=\left\{\left(i, p_{j} j\right)\right\}$ for some set of targets $\{j\}$, then let $M$ 's transitions at $i$ be exactly those of $B$.

Observe that $x_{i}^{*} \leq \sum_{j} p_{j} x_{j}^{*}+\delta_{i}$, since $\mathbf{x}^{*} \notin U_{B}^{+}$.

- If $B$ is nondeterministic, then there must be some target $j$ of $B$ such that $x_{i}^{*} \leq x_{j}^{*}+\delta_{i}$, again since $\mathbf{x}^{*} \notin U_{B}^{+}$. Let $M$ move from $i$ to $j$ with probability 1 .

Let $P=\left(p_{i j}\right)$ be the probability transition matrix of $M$. (It is a $k \times k$ matrix.) Since $H$ is stochastically acyclic, $M$ cannot have any recurrent classes other than the absorbing state $k$, and therefore the following system has a unique finite solution (this reasoning is at the heart of Lemma 5; see also [48]):

$$
\begin{aligned}
& x_{i}=\sum_{j=1}^{k} p_{i j} x_{j}+\delta_{i}, \quad \text { for } 1 \leq i<k ; \\
& x_{k}=0 .
\end{aligned}
$$

Moreover, one may obtain the solution to System (4) by iteration, starting from any initial seed for $x_{1}, \ldots, x_{k-1}$. (This is a standard result from Markov chains; it follows in particular from the theorem on p. 389 of [32].)

We will now iterate from two different seeds, obtaining contradictory results: 


\section{Iteration Scheme \#1:}

- Initialize $x_{i}^{(0)}=t_{i}$, for $i=1, \ldots, k$, where $\left\{t_{i}\right\}_{i=1}^{k}$ is the solution to System (3).

- For $m=0,1, \ldots$, iterate using the update rules:

$$
\begin{aligned}
x_{i}^{(m+1)} & =\sum_{j=1}^{k} p_{i j} x_{j}^{(m)}+\delta_{i}, \quad \text { for } 1 \leq i<k ; \\
x_{k}^{(m+1)} & =0 .
\end{aligned}
$$

We claim that $x_{i}^{(m)} \leq t_{i}$ for all $i=1, \ldots, k$ and all $m=0,1, \ldots$.

To see this:

The claim is certainly true for all $i$ when $m=0$ and also for $i=k$ for all $m$.

Inductively, suppose the claim holds for some $m \geq 0$. Then, with $1 \leq i<k$ :

$$
x_{i}^{(m+1)} \leq \sum_{j=1}^{k} p_{i j} t_{j}+\delta_{i} \leq t_{i} .
$$

The first inequality follows from the inductive hypothesis, the second from the fact that the right side of System (4) is a special case appearing in the maximizations of System (3).

Consequently, $\quad x_{i}=\lim _{m \rightarrow \infty} x_{i}^{(m)} \leq t_{i}, \quad$ for $i=1, \ldots, k$,

where $\left\{x_{i}\right\}_{i=1}^{k}$ is the solution to System (4).

\section{Iteration Scheme \#2:}

- Initialize $y_{i}^{(0)}=x_{i}^{*}$, for $i=1, \ldots, k$, where $\mathbf{x}^{*}$ is as supposed earlier.

- For $m=0,1, \ldots$, iterate using the update rules:

$$
\begin{aligned}
y_{i}^{(m+1)} & =\sum_{j=1}^{k} p_{i j} y_{j}^{(m)}+\delta_{i}, \quad \text { for } 1 \leq i<k ; \\
y_{k}^{(m+1)} & =0 .
\end{aligned}
$$

We claim that $y_{i}^{(m)} \geq x_{i}^{*}$ for all $i=1, \ldots, k$ and all $m=0,1, \ldots$.

Verifying:

Again, the claim is true for all $i$ when $m=0$ and also for $i=k$ for all $m$.

Inductively, suppose the claim holds for some $m \geq 0$. Then, with $1 \leq i<k$ :

$$
y_{i}^{(m+1)} \geq \sum_{j=1}^{k} p_{i j} x_{j}^{*}+\delta_{i} \geq x_{i}^{*} .
$$

The first inequality follows from the inductive hypothesis, the second from the construction of $M$.

Consequently, this time we see that

$$
x_{i}=\lim _{m \rightarrow \infty} y_{i}^{(m)} \geq x_{i}^{*}, \quad \text { for } i=1, \ldots, k .
$$


Combining the results of the two iteration schemes, we infer that $x_{i}^{*} \leq t_{i}$ for $i=1, \ldots, k$. In particular, for $i=\ell$, recalling the definition of $\mathbf{x}^{*}$, we see that

$$
t_{\ell}=0+t_{\ell}=x_{k}^{*}+t_{\ell}<x_{\ell}^{*} \leq t_{\ell}
$$

That says $t_{\ell}<t_{\ell}$, a contradiction.

\subsection{Characterizing Controllability with Spheres}

The following two theorems characterize topologically the ability of a finite discrete system to reach any state from any other state despite control uncertainty.

Theorem 11 (Graph Controllability) Let $G=(V, \mathcal{A})$ be a stochastic graph with $V \neq \emptyset$. The following two statements are equivalent:

(i) For every pair of states $v, u \in V, G$ contains a stochastic strategy for attaining $u$ from $v$.

(ii) $\Delta_{G} \simeq S^{n-2}$, with $n=|V|$.

Clarification: In (i), the strategy may depend on $v$ and $u$, that is, different pairs of states may give rise to different strategies.

Proof. If $V$ consists of a single state, then the empty simplex is a stochastic strategy for attaining that state from itself. In fact, that is the only possible stochastically acyclic collection of actions of $G$; any action of $G$ must be a self-loop. Consequently, $\Delta_{G}=\{\emptyset\}$, which by convention is the sphere of dimension -1 , i.e., $n-2$. So, henceforth we may assume that $V=[n]$, with $n \geq 2$.

\section{Proof that (i) implies (ii):}

We repeatedly use Lemma 9 to infer that $\bigcup_{A \in \mathcal{A}} U_{A}$ contains every point of $\mathbf{R}^{n}$ except the line on which all coordinates are equal. Thus $\Delta_{G} \simeq S^{n-2}$, by Theorem 8 .

II. Proof that (ii) implies (i):

Suppose (i) is false. Then there must be some $s \in V$ such that $G$ does not contain a complete stochastic strategy for attaining $s$ (on the state space $V$ ). Define a new stochastic graph $G_{+s}=\left(V, \mathcal{A}^{\prime}\right)$, where $\mathcal{A}^{\prime}$ is the union of $\mathcal{A}$ and all possible loopback actions at $s$. (This construction is similar to that appearing in the proof of Theorem 1, except that we have added loopbacks at $s$ rather than merely replaced the existing actions at $s$ with loopbacks.)

Since $G_{+s}$ contains all the actions of $G$,

$$
\bigcup_{A \in \mathcal{A}} U_{A} \subseteq \bigcup_{A \in \mathcal{A}^{\prime}} U_{A}
$$

By Theorem 8, $\bigcup_{A \in \mathcal{A}} U_{A} \simeq S^{n-2}$.

Using the fact that the covering sets $U_{A}$ are homogeneous and invariant with respect to translation along the line $\left\{\mathbf{x} \in \mathbf{R}^{n} \mid x_{1}=\cdots=x_{n}\right\}$, as well as the fact that no proper subset of a sphere is homotopic to that same sphere, one sees that $\bigcup_{A \in \mathcal{A}^{\prime}} U_{A} \simeq S^{n-2}$. 
On the other hand, a collapsibility argument very similar to that used in the proof of Theorem 1 shows that $\Delta_{G_{+s}}$ must be contractible. By Theorem $8, \bigcup_{A \in \mathcal{A}^{\prime}} U_{A}$ has the same homotopy type as $\Delta_{G_{+s}}$. That says $S^{n-2}$ is contractible, a contradiction.

Definition: A graph is fully controllable if, for any initial state and any stop state, the graph contains a stochastic strategy for attaining the stop state from the initial state.

Example: The strategy complex of the graph in Fig. 13 is homotopic to a circle, i.e., to $S^{n-2}$. Every action in the graph is uncertain, with either nondeterministic or stochastic outcomes. Nonetheless, Theorem 11 tells us that the graph is fully controllable.

Key Point: Despite significant control uncertainty, all states are precisely attainable.

The Power of Topology: One can easily verify the theorem's assertion by inspection for the example of Fig. 13. For instance, the system can be certain of attaining state 2 from state 1 via the strategy $\{1 \rightarrow \mathrm{p} 2, \mathrm{q} 3 ; 3 \rightarrow 1,2\}$. What we do not know is the exact path the system will take: It may move directly to state 2 from state 1 or it may move through state 3 . In fact, it may even cycle for a while between states 1 and 3 before moving to state 2 . This is exactly one of the properties we sought in the Introduction (see again page 5): When planning in the presence of uncertainty, one should focus not on specific trajectories but entire classes of motions. Topology is doing this naturally for us.

Here is a time-bounded version of Theorem 11:

Theorem 12 (Time-Bounded Graph Controllability) Let $G=(V, \mathcal{A})$ be a stochastic graph with $V \neq \emptyset$ and with associated nonnegative action transition times $\left\{\delta_{A}\right\}_{A \in \mathcal{A}}$. Let $T \geq 0$ be given. The following two statements are equivalent:

(i) For every pair of states $v, u \in V, G$ contains a stochastic strategy $\sigma_{v u}$ for attaining $u$ from $v$ with maximal worst-case expected convergence time $t_{\max }\left(\sigma_{v u}\right)$ no greater than $T$.

(ii) $\Delta_{G}^{T} \simeq S^{n-2}$, with $n=|V|$.

Proof. The proof is similar to that given for Theorem 11. One difference is that the covering sets $U_{A}^{+}$are not homogeneous (they are still invariant with respect to translation along the line in $\mathbf{R}^{n}$ on which all coordinates are equal). This fact is one reason Lemma 7 uses the cylinder $\partial C_{T+\epsilon}$.

Remark: Theorem 1 in Section 4 established a two-world scenario for nondeterministic loopback graphs: $\Delta_{G \leftarrow s}$ is homotopic either to $S^{n-2}$ or to a point, depending on whether $G$ contains a complete guaranteed strategy for attaining $s$, or not, respectively. The same result holds for stochastic loopback graphs, both in the general case and in the time-bounded case. In the time-bounded case, we associate transition time zero to each loopback action. Thus, $\Delta_{G_{\leftarrow s}}^{T}$ is homotopic either to $S^{n-2}$ or to a point. If $G$ contains a complete stochastic strategy for attaining $s$, all of whose worst-case expected convergence times are bounded by $T$, then $\Delta_{G_{\leftarrow s}}^{T}$ is homotopic to a sphere. Otherwise, $\Delta_{G_{\leftarrow s}}^{T}$ is homotopic to a point. 


\section{Topology as a Design Tool: An Example}

We are beginning to understand how the topology of a strategy complex reflects a system's capabilities. Theorem 11 tells us that homotopy equivalence between the system's strategy complex and a sphere of dimension two less than the number of system states is equivalent to full controllability.

Moreover, while it remains a research question to understand the full implications of homotopy equivalence, we will soon see that the strategy complex precisely characterizes the stochastically attainable and (potentially) unattainable goals of a system. Of course, full controllability means that all goals are stochastically attainable. In the absence of full controllability, the strategy complex still informs us about system capabilities, in a manner to be explained in the next section.

This section illustrates how a strategy complex may be used as a design tool: We turn design knobs while watching how the strategy complex changes, freezing the design when the strategy complex exhibits a desired topology.

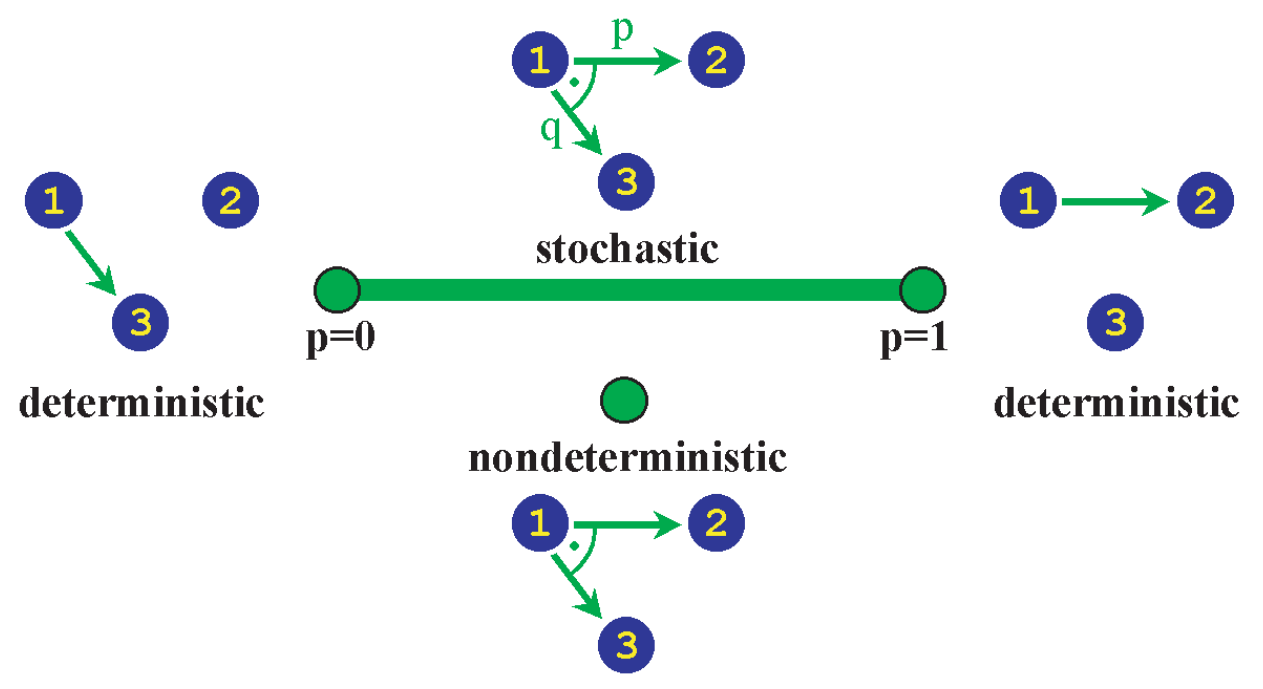

Figure 15: The parameter space for designing an action at state 1. The tuning parameter, drawn in green, consists of an interval and a point. The action is depicted, also in green, for four characteristic parameter values.

Let us revisit the three-state example of Fig. 13, but now consider the situation in which we are designing the actions and the control error. There is one action at each state. The action may be a deterministic transition to one of the other two states, or a stochastic motion to both those states, with tunable transition probabilities, or a nondeterministic motion to both those states. Fig. 15 shows the possible tuning parameter for the action at state 1.

\subsection{How Many Design Scenarios?}

Counting the design scenarios depends on one's perspective:

- Degrees of Freedom: The design problem is a three-degree-of-freedom problem. Each degree of freedom may be modeled as $[0,1] \cup\{\triangleleft\}$, where $[0,1]$ represents the 
stochastic continuum whose endpoints are the deterministic actions and $\triangleleft$ represents the nondeterministic action.

- Characteristic Cases: As the English description above suggests, there are four characteristic cases for each tuning parameter, so 64 characteristic cases overall. Ignoring symmetries, there are 16 cases.

Comment: Á priori, the precise transition probabilities for a stochastic action could be significant. They certainly affect convergence times, and thus the complexes $\Delta_{G}^{T}$. However, varying the probabilities does not affect the homotopy type of $\Delta_{G}$, except possibly at an endpoint when some transition probability goes to zero.

- Topologically: There are 8 distinct strategy complexes possible, as we will see shortly. In fact, ignoring symmetries, there are only 4 topologically distinct cases. In other words, from the perspective of overall system capabilities, as measured by stochastically attainable goals, this design problem entails a choice between four different systems.

\subsection{Tuning Convergence Times and Designing System Capabilities}

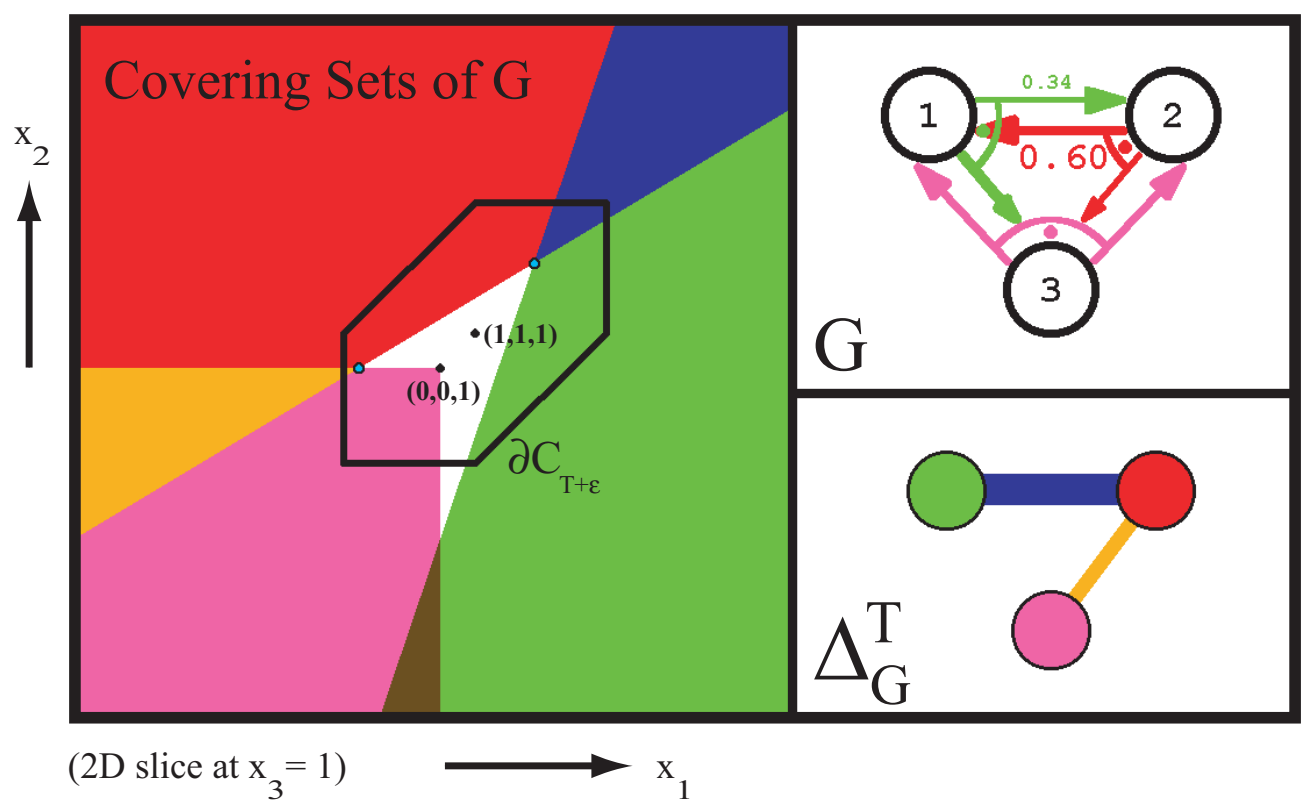

Figure 16: One may design system capabilities by tuning the topology of $\Delta_{G}^{T}$ : As the design parameters change, so do the covering sets associated with the graph's actions. The homotopy type of the cover intersected with $\partial C_{T+\epsilon}$ coincides with the homotopy type of the simplicial complex $\Delta_{G}^{T}$. This figure shows a snapshot of that process. As did Fig. 14, the figure shows a two-dimensional slice of the three-dimensional covering sets. See text for further details.

Color Legend: Corresponding graph actions, covering sets, and simplices are color-coded. In case color is not visible, placements are as in Figures 13 and 14.

Probabilities: The actions at states 1 and 2 are stochastic. To reduce clutter, the figure only shows the probabilities of moving from state 1 to 2 and vice-versa. 
Fig. 16 shows a snapshot of the tuning process. The upper right frame shows the graph and its actions for the current choice of tuning parameters, the big frame on the left shows the $\left(x_{1}, x_{2}\right)$-slice at $x_{3}=1$ of the actions' covering sets. As in Section 7 , each action's covering set gives rise to a vertex of the simplicial complex $\Delta_{G}^{T}$, shown in the lower right frame of the figure. Also drawn over the covering sets is a convergence time cylinder $\partial C_{T+\epsilon}$ for some desired convergence time $T$. Key intersection points of the covering sets within this desired time are highlighted. They give rise to the edges in the complex $\Delta_{G}^{T}$; the thickness of an edge is roughly proportional to the difference between the maximal convergence time of the edge (viewed as a stochastic strategy) and the maximal time $T$ permitted.

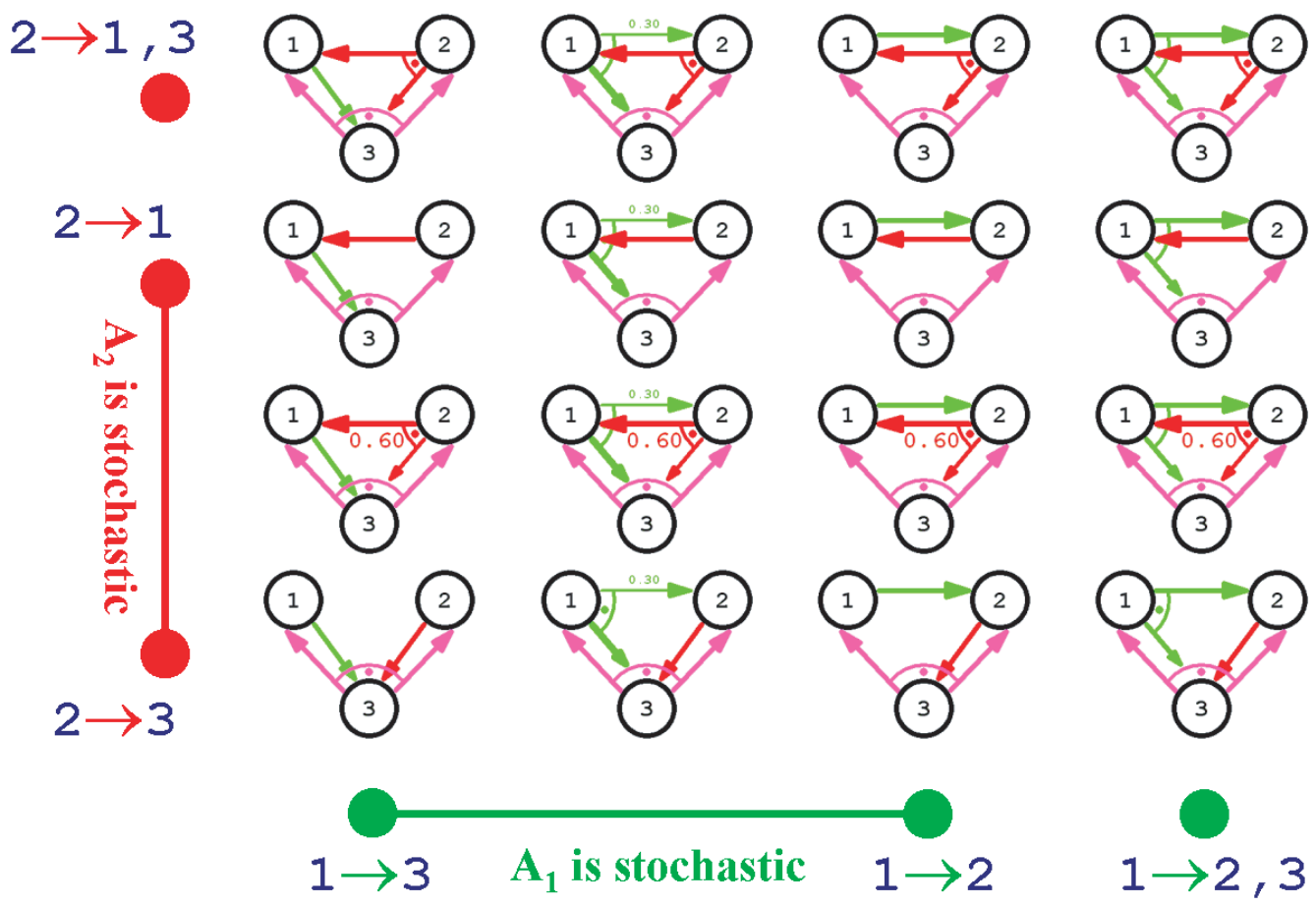

Figure 17: A slice of the design space from the perspective of the resulting stochastic graphs. $A_{i}$ means the action at state $i$. In the slice shown, $A_{3}$ is nondeterministic while the parameters for actions $A_{1}$ and $A_{2}$ vary over their full ranges. For the stochastic range of each parameter, the figure depicts a representative action. To reduce clutter, the figure only shows the probability of moving from state 1 to 2 or vice-versa. See Fig. 18 for the associated covering sets and Fig. 19 for the associated simplicial complexes.

Figures 17-19 depict a two-dimensional slice of the three-degree-of-freedom design space from three perspectives. In this slice, the tuning parameter for the action at state 3 is fixed to be nondeterministic, while the tuning parameters for the actions at states 1 and 2 vary. Fig. 17 shows how the graphs vary as the tuning parameters vary, Fig. 18 shows how the covering sets vary, and Fig. 19 shows how the simplicial complexes $\Delta_{G}$ vary (we now ignore the precise convergence times, focusing on $\Delta_{G}$ not $\Delta_{G}^{T}$ ). Since there are three actions without self-loops, $\Delta_{G}$ always contains all three actions as vertices. $\Delta_{G}$ cannot be the full triangle on those three vertices, since such a triangle would not be stochastically acyclic. Consequently, there should 


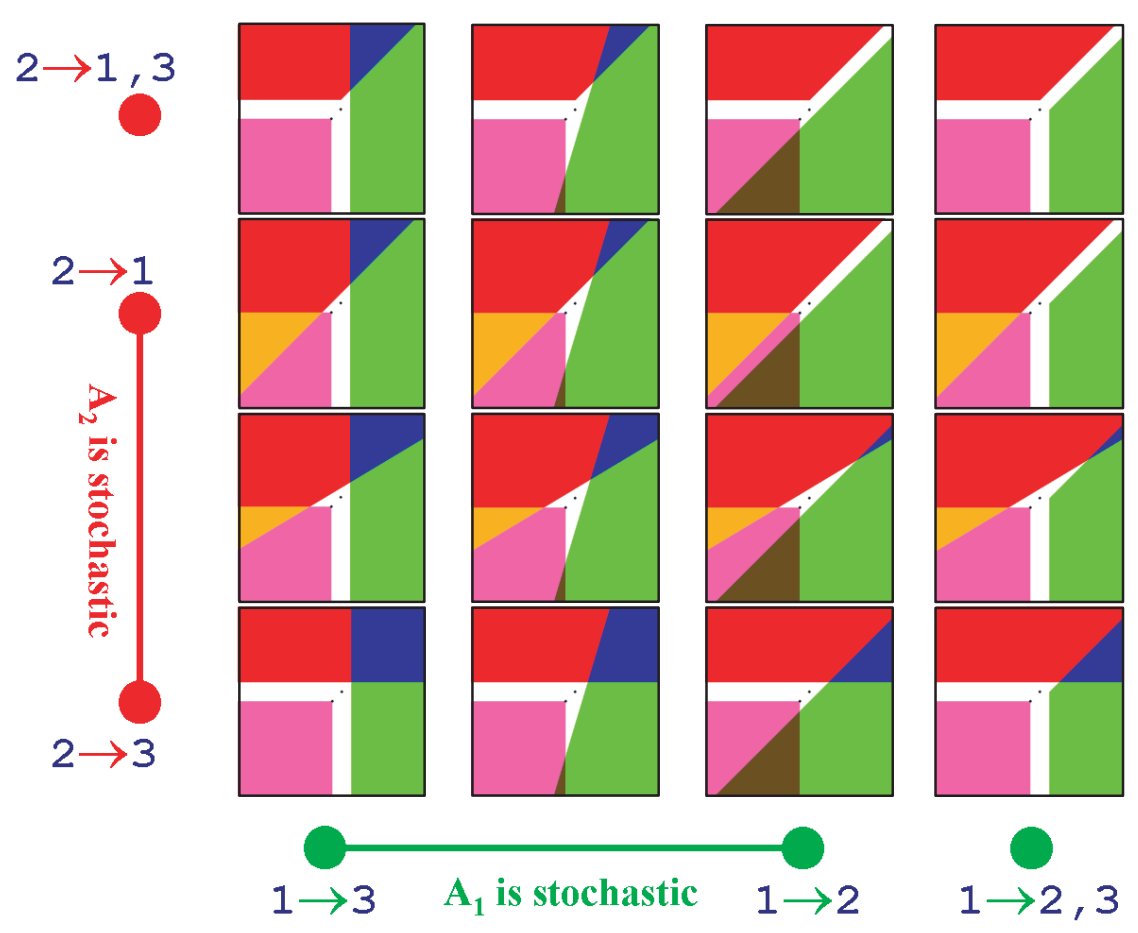

Figure 18: A slice of the design space from the perspective of the resulting covering sets. The covering sets shown correspond to the graphs of Fig. 17, assuming all actions have unit transition time.

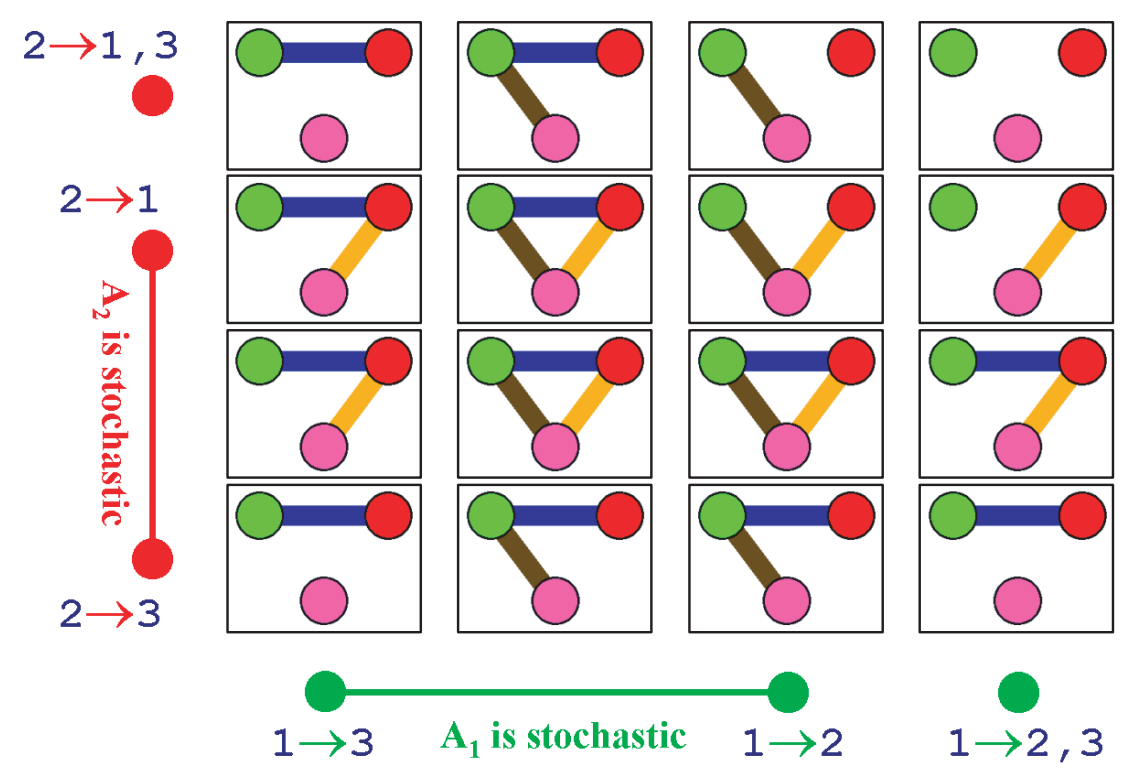

Figure 19: A slice of the design space from the perspective of the resulting simplicial complexes $\Delta_{G}$. The complexes shown correspond to the covering sets of Fig. 18. 


\begin{tabular}{|c|c|c|c|}
\hline $\begin{array}{l}\mathrm{SmN}, \mathrm{SSN}, \mathrm{pSN} \text {, } \\
\mathrm{mNS}, \mathrm{SNS}, \mathrm{mmS} \text {, } \\
\mathrm{SmS}, \mathrm{mSS}, \mathrm{SSS} \text {, } \\
\mathrm{pSS}, \mathrm{NSS}, \mathrm{mpS} \text {, } \\
\mathrm{SpS}, \mathrm{ppS}, \mathrm{NpS} \text {, } \\
\mathrm{SNp}, \mathrm{Smp}, \mathrm{SSp} \text {, } \\
\mathrm{pSp}, \mathrm{Spp}, \mathrm{ppp} \text {, } \\
\mathrm{mmm}, \mathrm{Smm}, \mathrm{mSm} \text {, } \\
\mathrm{SSm}, \mathrm{pSm}, \mathrm{NSm}\end{array}$ & $\mathrm{NNN}$ & 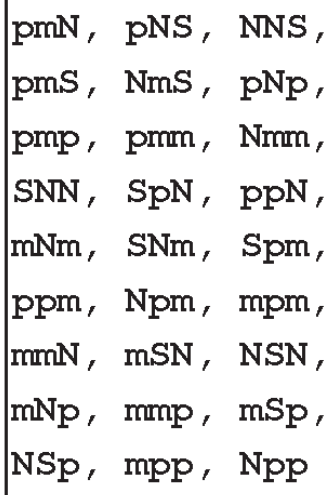 & $\begin{array}{l}\mathrm{mNN}, \mathrm{mpN}, \mathrm{NpN} \text {, } \\
\mathrm{NmN}, \mathrm{NNp}, \mathrm{Nmp} \text {, } \\
\mathrm{pNN}, \mathrm{pNm}, \mathrm{NNm}\end{array}$ \\
\hline
\end{tabular}

Figure 20: There are four topologically distinct simplicial complexes attainable by varying the design parameters of a three-state graph with one (acyclic) action at each state. These complexes describe the inherent capabilities of the system under varying degrees of control uncertainty. The columns classify the 64 characteristic design cases in terms of the complexes they generate. The leftmost column corresponds to full controllability. The example of Fig. 13 is circled.

Legend: Each characteristic case is summarized by a 3-letter code, with the $i^{\text {th }}$ letter describing the action at state $i$ as follows:

$$
\begin{aligned}
& \text { S: stochastic m: deterministic transition to state } i-1 \\
& \mathrm{~N} \text { : nondeterministic } \quad \mathrm{p} \text { : deterministic transition to state } i+1 \\
& \text { (with wraparound at } 3 / 1 \text { ) }
\end{aligned}
$$

be as many different complexes in the full design space as there are ways to form edges from three vertices, namely eight ways. Indeed, inspection of Fig. 19 reveals eight unique complexes; the full design space repeats these. Ignoring symmetries, there are in fact only four distinct complexes, shown in the top row of Fig. 20.

One may now classify each of the 64 characteristic cases of the design space by the type of its associated strategy complex $\Delta_{G}$, as shown in the columns of Fig. 20.

Section 10 discusses in more detail what each of the four complexes means. We already know that the complex defining the leftmost column constitutes full controllability. We thus see twenty-seven different characteristic implementations that attain full controllability. The example of Fig. 13 is circled. 
Design Implications: From a design perspective, one may now use other constraints to decide which of the twenty-seven implementations might be desirable for full controllability. Moreover, one may tune the convergence times using the idea described in Fig. 16. This process is much like choosing forces within the nullspace of a grasp to satisfy some design criterion while maintaining a specific equilibrium grasp on an object [51].

\section{Duality}

In order to understand better how the complex $\Delta_{G}$ describes system capabilities, we need to develop a perspective dual to the spherical perspectives of Theorems 1 and 11. In the process, we will see how backchaining and contractibility of certain subcomplexes are manifestations of the same idea. Finally, we will see how to match complexes with design criteria.

\subsection{Start Region Contractibility}

Consider the start region $V_{\sigma}$ of some simplex $\sigma$ of stochastically acyclic actions in a graph $G$. As we will see in this section, the subcomplex induced by all actions of the graph with sources in that region is contractible. Intuitively, contractibility is consistent with viewing $\sigma$ as a collapsing of $V_{\sigma}$, moving the system off those states.

Definitions Suppose $G=(V, \mathcal{A})$ is a stochastic graph and $W \subseteq V$. Define the following:

- $\mathcal{A} \mid W$ is the collection of all actions of $G$ whose sources lie in $W$.

- $W_{\mathcal{A}}$ is the union of $W$ and all targets of actions in $\mathcal{A} \mid W$.

- $G \mid W=\left(W_{\mathcal{A}}, \mathcal{A} \mid W\right)$. Intuition: $G \mid W$ is a subgraph of $G$, induced by all the actions of $G$ whose sources lie in $W$ but whose targets may lie outside of $W$.

- A moves off $W$ if $A \in \mathcal{A} \mid W$ and one of the following is true:

(i) $A$ is stochastic with at least one of its targets in $V \backslash W$, or

(ii) $A$ is nondeterministic with all of its targets in $V \backslash W$.

Lemma 13 (Contractibility of Start Regions) Let $G=(V, \mathcal{A})$ be a stochastic graph. Suppose $W=V_{\sigma}$ for some $\sigma \in \Delta_{G}$. Then $\Delta_{G \mid W}$ is contractible.

Proof. If $W=\emptyset$, then $\Delta_{G \mid W}$ is the void complex $\emptyset$, which is considered contractible [47] (do not confuse it with the empty complex $\{\emptyset\}$ ).

If $W \neq \emptyset$, then there there is some action $A \in \mathcal{A} \mid W$ that moves off $W$. To see this, suppose otherwise. Imagine constructing a Markov chain $M$ with support $W$ from the graph $(V, \sigma)$ via the process of page 22. If no action $A$ moves off $W$, then an adversary can ensure that $M$ has no transitions to states outside of $W$. This means $M$ must contain a recurrent class within $W$, since the chain is finite [32]. That contradicts the stochastic acyclicity of $\sigma$ (recall Lemma 5).

Now suppose $\tau \in \Delta_{G \mid W}$. Consider $\tau^{\prime}=\tau \bigcup\{A\}$. If $\tau^{\prime}$ is not stochastically acyclic, then $\tau^{\prime}$ must give rise to some Markov chain $\left(M, W^{\prime}\right)$ whose support $W^{\prime}$ is a recurrent class. Since 
$\tau$ is stochastically acyclic, $M$ must include transitions induced by $A$. Since $A$ moves off $W$ it moves off $W^{\prime}$, contradicting the assumed recurrent nature of $W^{\prime}$.

Consequently, $\tau^{\prime}$ is stochastically acyclic, establishing that $\Delta_{G \mid W}$ is a cone with apex $A$, hence contractible.

Remark: A similar result holds for the time-bounded case. The proof is more involved. The problem in the time-bounded case is that the action $A$ appearing in the proof above need no longer be a cone apex for the complex $\Delta_{G \mid W}^{T}$. The reason is that some worst-case expected convergence time of $\tau^{\prime}$ may exceed the desired bound $T$, even though $\tau$ and $A$ separately satisfy the bound. One must therefore argue differently. In particular, one can return to the covering set approach and see that the cover corresponding to $\Delta_{G \mid W}^{T}$ must be contractible.

\subsection{Source Complex}

This section associates a new simplicial complex to every graph, called the source complex, in effect compressing the graph's strategy complex. The main result is that compression preserves homotopy type. This result explains a slight sleight of hand in the discussion of Section 9. Even though strategy complexes reside in a space of actions, we managed to view the complexes as sitting on the original graph. Doing so was very natural in the example of Section 9 since the graph contained exactly one action at each state. The current section establishes that one may always view the strategy complex as residing back on the graph, via the compression to source complexes.

Definition: The source complex $\bar{\Delta}_{G}$ of a stochastic graph $G=(V, \mathcal{A})$ is the collection $\left\{V_{\sigma} \mid \sigma \in \Delta_{G}\right\}$. The underlying vertex set of $\bar{\Delta}_{G}$ is $V$.

It is easy to check that $\bar{\Delta}_{G}$ really is a simplicial complex.

$\bar{\Delta}_{G}$ describes all possible start regions of strategies definable by actions of $G$. The complements of those start regions, that is, sets of the form $V \backslash V_{\sigma}$, describe all stochastically attainable goals. Observe that $V$ is never a simplex of $\bar{\Delta}_{G}$, since any purported strategy with actions at all states of a graph would actually cycle forever.

Theorem 14 (Compression Preserves Homotopy Type) Let $G$ be a stochastic graph.

$$
\Delta_{G} \simeq \bar{\Delta}_{G}
$$

Proof. Let $P=\mathcal{F}\left(\Delta_{G}\right)$ and $Q=\mathcal{F}\left(\bar{\Delta}_{G}\right)$ be the face posets of the two complexes.

Define $f: P \rightarrow Q$ by $f(\sigma)=V_{\sigma}$. Suppose $W \in Q$. Then $f^{-1}\left(Q_{\leq W}\right)$ is the face poset of $\Delta_{G \mid W}$, which is contractible by Lemma 13. The desired result now follows from the Quillen Fiber Lemma.

Remark: Again, a similar result holds for the time-bounded case.

The following lemma is a useful tool:

Lemma 15 (Source Complex Membership) Let $G=(V, \mathcal{A})$ be a stochastic graph. Suppose $W$ is a nonempty proper subset of $V$ such that every proper subset of $W$ is a simplex in $\bar{\Delta}_{G}$. Then $W \in \bar{\Delta}_{G}$ if and only if $G$ contains an action that moves off $W$. 
Proof. One direction follows from the proof of Lemma 13.

For the other direction, suppose $A$ moves off $W$. Let $v$ be the source of $A$. By assumption, there is some $\sigma \in \Delta_{G}$ such that $V_{\sigma}=W \backslash\{v\}$. Consider $\tau=\sigma \bigcup\{A\} . \tau$ is stochastically acyclic since $\sigma$ is stochastically acyclic and since any transitions at $v$ induced by $A$ in a Markov chain derived from $\tau$ have probability less than 1 of remaining in $W$. $V_{\tau}=W$, so $W \in \bar{\Delta}_{G}$.

\subsection{Contractibility Characterization of Goal Attainability}

We now obtain a result dual to Theorem 1. The proof of the following theorem makes explicit the connection between backchaining and contractibility.

Definition: Suppose $\Sigma$ is a simplicial complex with underlying vertex set $V$. A minimal nonface of $\Sigma$ is a set $W \subseteq V$ such that $W$ is not a simplex of $\Sigma$ but every proper subset of $W$ is a simplex of $\Sigma$.

\section{Theorem 16 (Contractibility Characterization of Goal Attainability)}

Suppose $G=(V, \mathcal{A})$ is a stochastic graph and $\emptyset \neq S \subseteq V . S$ is a stochastically attainable goal in $G$ if and only if $\Delta_{G \mid W}$ is contractible for every $W \subseteq V \backslash S$.

Proof. I. Suppose $G$ contains a complete stochastic strategy for attaining $S \subseteq V$.

Then there is some $\sigma \in \Delta_{G}$ such that $V_{\sigma}=V \backslash S$. By Lemma 13, and the simplicial nature of $\sigma$, we see that $\Delta_{G \mid W}$ is contractible for every $W \subseteq V_{\sigma}$.

II. Suppose $\Delta_{G \mid W}$ is contractible for every $W \subseteq V \backslash S$.

We now construct $\sigma \in \Delta_{G}$ by backchaining:

1. Initialize $S^{*}:=S$ and $\sigma:=\emptyset$.

2. If $S^{*}=V$, then done; return $\sigma$.

3. Otherwise, as shown below, $G$ contains an action $A$ that moves off $V \backslash S^{*}$.

Let $v$ be the source of $A$. Update $S^{*}:=S^{*} \cup\{v\}$ and $\sigma:=\sigma \bigcup\{A\}$.

4. Repeat Steps 2 and 3 until done.

It is easy to see that the $\sigma$ returned in Step 2 really is stochastically acyclic. $V_{\sigma}=V \backslash S$, so $\sigma$ is a complete stochastic strategy for attaining $S$, as desired.

To complete the proof, we need to establish the existence of action $A$ in Step 3. Suppose no such $A$ exists. By the proof of Lemma 13, this means $V \backslash S^{*} \notin \bar{\Delta}_{G}$. Choose nonempty $W \subseteq V \backslash S^{*}$ such that $W \notin \bar{\Delta}_{G}$ but every proper subset of $W$ is a simplex of $\bar{\Delta}_{G}$. In other words, $W$ is a minimal nonface of $\bar{\Delta}_{G}$. Observe that $W$ is also a minimal nonface of $\bar{\Delta}_{G \mid W}$.

$A$ priori, the underlying vertex set of $\bar{\Delta}_{G \mid W}$ is the state space $W_{\mathcal{A}}$ of $G \mid W$, but no state outside $W$ can be a vertex of $\bar{\Delta}_{G \mid W}$ since $G \mid W$ contains no actions at such states. Consequently, $W$ being a minimal nonface, $\bar{\Delta}_{G \mid W}$ is the boundary complex of the full simplex on $W$. Thus $\bar{\Delta}_{G \mid W} \simeq S^{|W|-2}$. On the other hand, Theorem 14 implies $\bar{\Delta}_{G \mid W} \simeq \Delta_{G \mid W}$, which is contractible. Contradiction. 


\subsection{The Dual Complex}

In algebraic topology, geometric duality becomes algebraic duality. The theorems are often formulated for nonempty proper subsets of spheres. Given the importance of spheres when reasoning about strategy complexes, one imagines that duality might help illuminate the homotopy type of a strategy complex. There is a simple combinatorial description for simplicial complexes of a particular duality known as Alexander Duality [7]. Formally, given a simplicial complex $\Sigma$ with underlying vertex set $V$, its Combinatorial Alexander Dual is the complex $\Sigma^{*}=\{\sigma \subseteq V \mid V \backslash \sigma \notin \Sigma\}$. Observe that $\left(\Sigma^{*}\right)^{*}=\Sigma$.

The Alexander dual of a source complex has an important natural meaning: it describes all goals that are not stochastically attainable (in the sense of page 30 ), as follows.

Definition: The dual complex $\bar{\Delta}_{G}^{*}$ of a stochastic graph $G=(V, \mathcal{A})$ is the collection $\left\{V^{*} \subseteq V \mid V \backslash V^{*} \notin \bar{\Delta}_{G}\right\}$. The underlying vertex set of $\bar{\Delta}_{G}^{*}$ is again $V$.

It is again easy to verify that $\bar{\Delta}_{G}^{*}$ is a simplicial complex. Moreover, it follows from the definitions that $V^{*} \in \bar{\Delta}_{G}^{*}$ if and only if there is no strategy $\sigma \in \Delta_{G}$ such that $V \backslash V_{\sigma} \subseteq V^{*}$. In

other words, we may think of $\bar{\Delta}_{G}^{*}$ as encoding all the potentially unattainable goals, meaning there is no stochastic strategy for attaining such a goal from everywhere in the graph.

The reader may be wondering why we have not also defined a simplicial complex to define the stochastically attainable goals. The answer is that simplicial complexes must satisfy some kind of monotone property: subsets of simplices must also be simplices. Failure to attain a goal is a monotone property; if we cannot be certain of attaining a goal of some size when planning with uncertainty, then we also cannot be certain of attaining any subset of that goal. In contrast, attainability is not similarly monotone; just because we can attain some goal does not mean we can attain a more precisely defined goal.

In short, start regions and potentially unattainable goals define simplicial complexes via the source and dual complexes. The stochastically attainable goals are given implicitly, as the complements of the start regions.

\subsection{Duality in Design}

We now understand better the topological information encoded in a graph's strategy complex. Earlier (Fig. 11) we saw that homotopy equivalence naturally favors precise actions over imprecise actions. We also realized (page 35) that topology abstracts away from particular trajectories to broad classes of motions. The source and dual complexes make this explicit. Moreover, the state space $V$ of a graph provides a good reference frame onto which homotopy equivalences should map. One can compare different system capabilities of different graphs with underlying state space $V$ by comparing their source (and/or dual) complexes.

Returning to the design problem of Section 9, Fig. 21 now shows both the source and dual complexes corresponding to Fig. 20. Moreover, the figure makes sense even if there are multiple actions or design parameters at each state.

Recall the various semantics we have been attaching to complexes. Consider the first column of complexes in Fig. 21. Previously we observed that this column corresponds to full controllability since the strategy complex is homotopic to a circle. That statement still holds, 


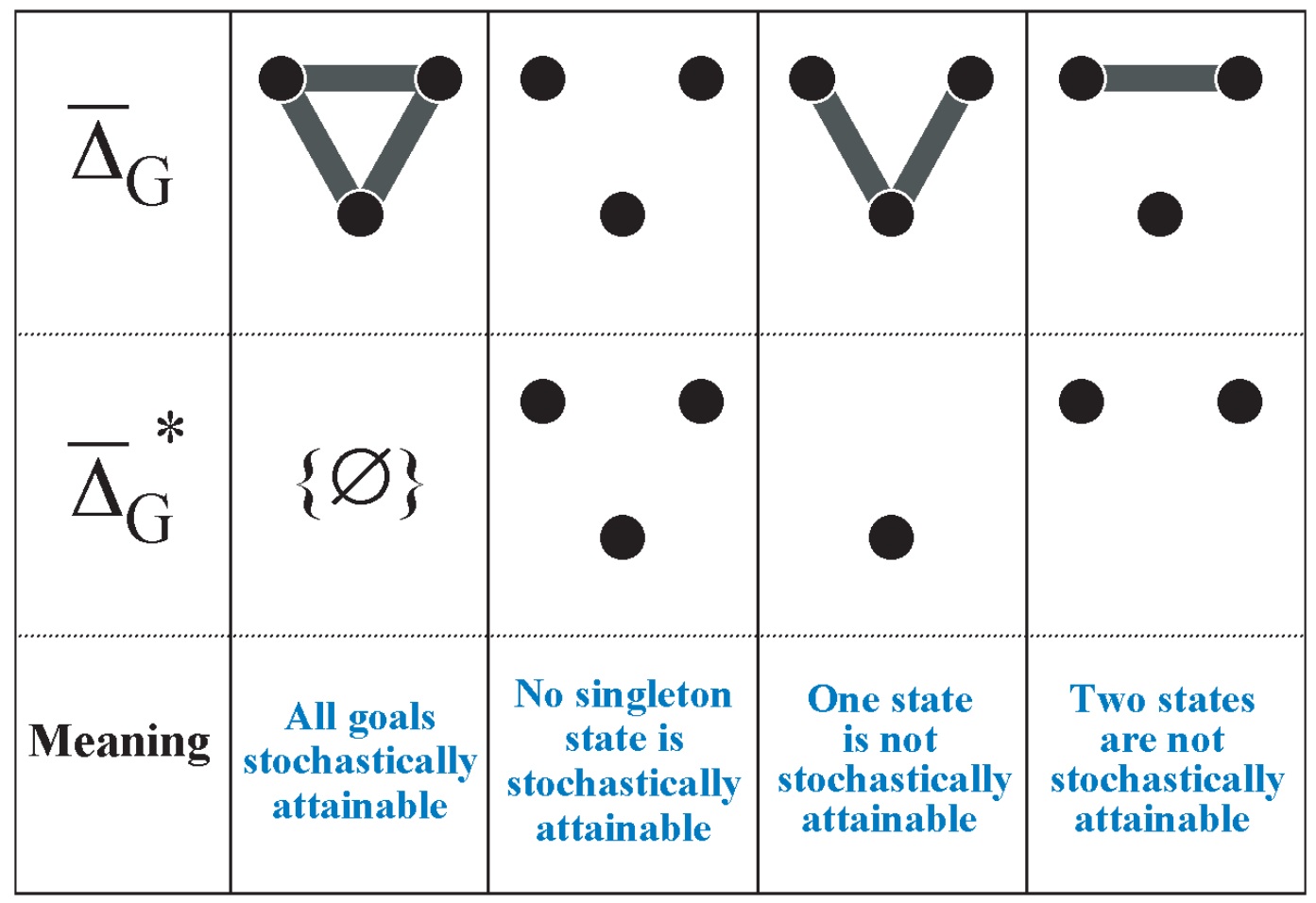

Figure 21: A comparison of the source and dual complexes for the design problem of Fig. 20.

only now we may refer to the source complex, since we now know that the strategy and source complexes have the same homotopy type. The dual complex in this case is the empty complex, meaning that there are no potentially unattainable goals. That statement is consistent with full controllability: all goals are stochastically attainable.

Remark: The reader may observe that this reasoning provides the basis for a purely combinatorial proof of Theorem 11, made possible by Theorem 14 and the semantics of source and dual complexes.

In some cases, one may not want full controllability. For instance, imagine that we are designing the hallways and door controllers in a bank. Suppose, for simplicity, there are three states: Outside, LobBy, and Vault. We might want to ensure that Outside and LobBy are reachable from everywhere by everyone, but that VAULT is reachable only by designated people. This suggests a system whose capabilities switch between those given by the first and third columns of complexes in Fig. 21, depending on the people walking in the hallways. We could go back to Fig. 20 to select a particular implementation of these switchable capabilities that also avoids passing by the VAULT accidentally. For instance, we might use a switchable deterministic action in the LOBBY whose actual direction is under the control of the bank's security guards. Finally, we might also go back to Fig. 16 to fine-tune convergence times. 


\section{Modularity}

This section discusses combination and simplification of graphs.

First, some topology. The (topological) join $X * Y$ of two topological spaces $X$ and $Y$ is the quotient space obtained from $X \times Y \times[0,1]$ by identifying each set $\{x\} \times Y \times\{0\}$ to a point and each set $X \times\{y\} \times\{1\}$ to a point, for all $x \in X$ and $y \in Y$ [61]. Geometrically, one may think of $X * Y$ as the union of all line segments joining points in $X$ to points in $Y$ [39]. For instance, the topological join of two finite disjoint edges is a tetrahedron.

In the case of simplicial complexes, the join assumes a simple combinatorial form:

Definition: Suppose $\Sigma$ and $\Gamma$ are two simplicial complexes with disjoint underlying vertex sets. Their (simplicial) join is the simplicial complex defined as

$$
\Sigma * \Gamma=\{\sigma \bigcup \gamma \mid \sigma \in \Sigma \text { and } \gamma \in \Gamma\}
$$

For instance, if $\Sigma$ and $\Gamma$ represent two disjoint line segments, say

$$
\begin{aligned}
& \Sigma=\left\{\emptyset,\left\{p_{1}\right\},\left\{p_{2}\right\},\left\{p_{1}, p_{2}\right\}\right\}, \\
& \Gamma=\left\{\emptyset,\left\{q_{1}\right\},\left\{q_{2}\right\},\left\{q_{1}, q_{2}\right\}\right\},
\end{aligned}
$$

then

$$
\begin{aligned}
\Sigma * \Gamma=\{\emptyset, & \left\{p_{1}\right\},\left\{p_{2}\right\},\left\{q_{1}\right\},\left\{q_{2}\right\}, \\
& \left\{p_{1}, p_{2}\right\},\left\{q_{1}, q_{2}\right\},\left\{p_{1}, q_{1}\right\},\left\{p_{1}, q_{2}\right\},\left\{p_{2}, q_{1}\right\},\left\{p_{2}, q_{2}\right\}, \\
& \left.\left\{p_{1}, p_{2}, q_{1}\right\},\left\{p_{1}, p_{2}, q_{2}\right\},\left\{p_{1}, q_{1}, q_{2}\right\},\left\{p_{2}, q_{1}, q_{2}\right\},\left\{p_{1}, p_{2}, q_{1}, q_{2}\right\}\right\},
\end{aligned}
$$

which represents a tetrahedron.

Observe: $\Sigma *\{\emptyset\}=\{\emptyset\} * \Sigma=\Sigma$ and $\Sigma * \emptyset=\emptyset * \Sigma=\emptyset$, for all simplicial complexes $\Sigma$.

Fact: Homotopy equivalence $(\simeq)$ commutes with the join operator $(*)$.

\subsection{Graph Union}

Suppose $G_{1}=\left(V_{1}, \mathcal{A}_{1}\right)$ and $G_{2}=\left(V_{2}, \mathcal{A}_{2}\right)$ are two stochastic graphs. (The state spaces $V_{1}$ and $V_{2}$ are allowed to overlap.)

Consider the stochastic graph $G=(V, \mathcal{A})$ representing the union of $G_{1}$ and $G_{2}$. It is defined by $V=V_{1} \cup V_{2}$ and $\mathcal{A}=\mathcal{A}_{1} \sqcup \mathcal{A}_{2}$. The operator "ப" means "disjoint union", that is, we treat actions in $\mathcal{A}_{1}$ and $\mathcal{A}_{2}$ as distinct even if they happen to have identical edge sets. (Any resulting redundancy washes away under homotopy equivalence, as we saw in Section 5.5 and Fig. 11.) We write $G=G_{1} \cup G_{2}$ for shorthand.

We wish to understand the relationship between $\Delta_{G}, \Delta_{G_{1}}$, and $\Delta_{G_{2}}$. Let us assume that $V_{1}$ and $V_{2}$ are both nonempty. Treating $\mathcal{A}_{1}$ and $\mathcal{A}_{2}$ as disjoint means $\Delta_{G_{1}}$ and $\Delta_{G_{2}}$ have disjoint underlying vertex sets, so we can form their join. Observe that $\Delta_{G} \subseteq \Delta_{G_{1}} * \Delta_{G_{2}}$, in fact,

$$
\Delta_{G}=\left\{\tau \in \Delta_{G_{1}} * \Delta_{G_{2}} \mid \tau \text { is stochastically acyclic }\right\} .
$$


The next lemma describes some common cases in which there is equality of complexes. Case (a) covers completely disjoint graphs, case (b) covers graphs that touch at a single state, and case (c) covers graphs in which one graph is essentially feeding into the other.

Lemma 17 (Join Sufficiency) Suppose $G=G_{1} \cup G_{2}$, with notation as above. Assume that $V_{1}$ and $V_{2}$ are both nonempty. In each of the following cases, $\Delta_{G}=\Delta_{G_{1}} * \Delta_{G_{2}}$ :

(a) $V_{1} \cap V_{2}=\emptyset$,

(b) $V_{1} \cap V_{2}$ contains a single state,

(c) $G_{1}$ (or $G_{2}$ ) has no actions with sources in $V_{1} \cap V_{2}$.

Proof. (a) Clear from the definitions. (Also, (a) follows from (c).)

(b) If $\sigma \bigcup \gamma$ is not stochastically acyclic although $\sigma \in \Delta_{G_{1}}$ and $\gamma \in \Delta_{G_{2}}$, then $\sigma \bigcup \gamma$ must induce a Markov chain $(M, W)$ whose support $W$ is a recurrent class containing the common state of $V_{1}$ and $V_{2}$. The transitions of $M$ at that state are induced by a single action $A$, with either $A \in \sigma$ or $A \in \gamma$. In the first case, all of $A$ 's targets lie in $V_{1}$ and so $\sigma$ alone could generate $(M, W)$, contradicting the stochastic acyclicity of $\sigma$. Similarly for the second case.

(c) Suppose the sources of all of $G_{1}$ 's actions lie in $V_{1} \backslash V_{2}$. If $\sigma \bigcup \gamma$ is not stochastically acyclic although $\sigma \in \Delta_{G_{1}}$ and $\gamma \in \Delta_{G_{2}}$, then $\sigma \bigcup \gamma$ must induce a Markov chain $(M, W)$ whose support $W$ is a recurrent class overlapping both $V_{1} \backslash V_{2}$ and $V_{2}$. Any action with source in $V_{2}$ must be an action of $\gamma$ and thus has no targets in $V_{1} \backslash V_{2}$, contradicting the assumption that $W$ is a recurrent class of $M$.

\subsection{Testing Acyclicity}

Suppose $G_{1}$ and $G_{2}$ do not satisfy the conditions of Lemma 17. The following options for computing $\Delta_{G}$ exist:

1. One possibility is to compute $\bar{\Delta}_{G}$ rather than $\Delta_{G}$. Conceptually, $\bar{\Delta}_{G}$ is easier to compute than $\Delta_{G}$. The two complexes have the same homotopy type (Theorem 14). Moreover, $\bar{\Delta}_{G}$ is more explicitly useful in characterizing system capabilities, as we saw in Section 10. Computing $\bar{\Delta}_{G}$ amounts to repeated backchaining (see Section 12).

2. Another possibility is to work with the definitions directly. For instance, in deciding whether a potential simplex $\sigma \bigcup \gamma$ really is stochastically acyclic, one may attempt to solve System (1) (written out for $\sigma \bigcup \gamma$ ) using strictly positive action transition times $\left\{\delta_{A}\right\}$. The solution will diverge precisely when $\sigma \bigcup \gamma$ can induce a Markov chain whose support is a recurrent class.

3. Finally, a third possibility is to break the computations into simpler pieces using the tools described next.

Lemma 18 (Combining Actions) Let $G=(V, \mathcal{A})$ be a stochastic graph and $\sigma \in \Delta_{G}$. Suppose there are actions $A_{1}, \ldots, A_{k} \in \mathcal{A}$, all with the same source, such that $\sigma \bigcup\left\{A_{i}\right\} \in \Delta_{G}$ for each individual action $A_{i}, i=1, \ldots, k$. Then $\sigma \bigcup\left\{A_{1}, \ldots, A_{k}\right\} \in \Delta_{G}$. 
Proof. Let $v$ be the source of the actions $A_{1}, \ldots, A_{k}$.

Any Markov chain $(M, W)$ induced by $\sigma \bigcup\left\{A_{1}, \ldots, A_{k}\right\}$ whose support $W$ is a recurrent class must have transitions at $v$ induced by some $A_{i}$. But then $\sigma \bigcup\left\{A_{i}\right\}$ could induce the same Markov chain. Contradiction.

Now suppose $\sigma \in \Delta_{G}$ and $A$ is an action of $G$. To avoid trivialities, assume $A$ is not a self-looping (non)deterministic action. Let $v_{A}$ be $A$ 's source. One can decide whether $\sigma \bigcup\{A\} \in \Delta_{G}$ as follows:

- By Lemma 18, one may assume without loss of generality that $v_{A} \notin V_{\sigma}$ (recall $V_{\sigma}$ is the set of all sources of actions in $\sigma$ ).

- By Lemma 17(c), if $v_{A}$ is not the target of some action of $\sigma$, or if no target of $A$ lies in $V_{\sigma}$, then $\sigma \bigcup\{A\} \in \Delta_{G}$. (This step is not necessary but provides a convenient filter.)

- Now consider the following system of equations:

$$
\begin{aligned}
& q_{v}=\max \left(\begin{array}{c}
\max _{B \in \sigma}\left(\max _{j} q_{u_{j}}\right), \quad \begin{array}{c}
B \in \sigma \\
B=\left\{\left(v, p_{j} u_{j}\right)\right\}
\end{array} \\
B=\left\{\left(v, u_{j}\right)\right\}
\end{array}\right) \text {, if } v \in V_{\sigma} ; \\
& q_{v}=1, \quad \text { if } v=v_{A} ; \\
& q_{v}=0, \quad \text { otherwise. }
\end{aligned}
$$

This system has a unique finite solution $\left\{q_{v}\right\}_{v \in V}$, since $\sigma$ is stochastically acyclic. One can compute the solution using iteration. For each $v \in V, q_{v}$ is the maximum probability under all Markov chains induced by $\sigma$ that the system, when started at state $v$, will reach $v_{A}$, the source of action $A$.

Consequently:

(i) If $A$ is stochastic then $\sigma \bigcup\{A\} \in \Delta_{G}$ if and only if $q_{u}<1$ for some target $u$ of $A$.

(ii) If $A$ is nondeterministic, then $\sigma \bigcup\{A\} \in \Delta_{G}$ if and only if $q_{u}<1$ for all targets $u$ of $A$.

\subsection{Simplification via Strongly Controllable Subspaces}

For regular directed graphs, one defines an equivalence relation by saying that two states are equivalent if each is reachable from the other. The resulting equivalence classes are called strongly connected components. One may form a quotient graph by collapsing each strongly connected component to a single point. Ignoring induced self-loops, the result is a directed acyclic graph.

This section pursues such simplification for stochastic graphs. We will discover some enrichments not present in regular directed graphs. 

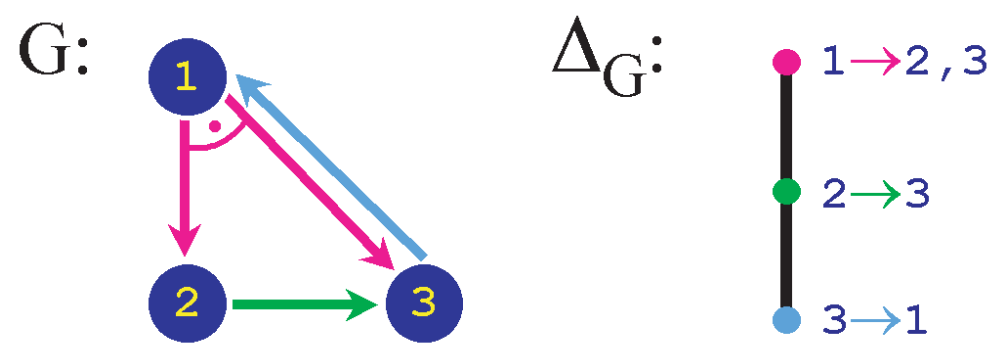

Figure 22: States 1 and 3 are each certainly attainable from the other, but an excursion to state 2 cannot be either excluded or forced by the system when leaving state 1. (An adversary may have control over the precise path.)

\section{An Equivalence Relation}

The first subtlety concerns connectivity. It is not enough to define two states as equivalent if each is reachable from the other. For instance, in Fig. 22, there exist strategies for attaining state 3 from state 1 and vice-versa. The problem is that the strategy to attain 3 from 1 might or might not pass through state 2 . That would be fine if the graph contained strategies for purposefully attaining state 2 from 1 and 3 , but it does not.

We therefore need a stronger requirement than pairwise reachability. Here it is:

Definitions Suppose $G=(V, \mathcal{A})$ is a stochastic graph.

Define a binary relation $\leftrightarrow$ on $V \times V$ as follows:

- $v \leftrightarrow w$ if $G$ contains a subgraph $H=(W, \mathcal{B})$ such that:

(a) $v, w \in W, \quad$ and

(b) $\Delta_{H} \simeq S^{|W|-2}$.

It is easy to see that $\leftrightarrow$ defines an equivalence relation on $V$. We refer to an equivalence class of $\leftrightarrow$ as a strongly controllable subspace of $G$.

For each equivalence class $W$ there is some subgraph $H=(W, \mathcal{B})$ whose strategy complex is homotopy equivalent to a sphere of dimension $|W|-2$. The proof of Theorem 11 ensures that $H$ contains strategies for attaining any state in $W$, without leaving $W$.

- If $\sim$ is any equivalence relation on $V$, let $G / \sim$ be the quotient graph obtained by collapsing states to their equivalence classes. Thus $G / \leftrightarrow$ collapses the strongly controllable subspaces of $G$.

A special case: Let $H=(W, \mathcal{B})$ be a subgraph of $G$ with $W \neq \emptyset$. Define the equivalence as follows: Each state of $V \backslash W$ is equivalent only to itself, while all states in $W$ are equivalent. This means $W$ collapses to a point, call it $\diamond$. We designate the resulting quotient graph by $G / W . G / W=\left(V^{\prime}, \mathcal{A}^{\prime}\right)$, with $V^{\prime}=V \backslash W \bigcup\{\diamond\}$ and $\mathcal{A}^{\prime}$ essentially identical to $\mathcal{A}$ except that any states of $W$ appearing as sources or targets in $\mathcal{A}$ have been remapped to $\diamond$ in $\mathcal{A}^{\prime}$.

Side issues: Distinct actions of $G$ may effectively become the same action in $G / \sim$. Nonetheless, we treat them as distinct. In other words, there is a bijective correspondence between $\mathcal{A}$ and $\mathcal{A}^{\prime}$. Once again, any redundancy will be washed away by homotopy equivalence 
once we pass to strategy complexes. The reader may also notice that for a given action, different targets in $G$ may become the same target in $G / \sim$. This poses no real issue. Nondeterministic actions are sets of edges, so the redundancy disappears automatically. For stochastic actions, one can add up the probabilities of different edges with the same target to form a single edge. Finally, some actions that are stochastically acyclic in $G$ may very well become selflooping (non)deterministic actions in $G / \sim$. Such actions disappear when we pass to strategy complexes.

Now imagine that $H=(W, \mathcal{B})$ is a subgraph of $G$ satisfying $\Delta_{H} \simeq S^{|W|-2}$ (W need not be an equivalence class of $G$ under $\leftrightarrow$, but must certainly be a subset of such an equivalence class). Then the system has full controllability within $H$, by Theorem 11 . Consequently, $H$ and $G / W$ are almost separate graphs that touch at a single state, as in Lemma 17(b). We might therefore expect to see equality between complexes as in that lemma. This analogy is not quite correct; the single common state is $\diamond$ in $G / W$, but it might be all of $W$ in $H$. As a result, equality becomes homotopy equivalence, as the following lemma and its proof demonstrate.

Lemma 19 (Factoring Controllable Subgraphs) Let $G=(V, \mathcal{A})$ be a stochastic graph and $H=(W, \mathcal{B})$ a subgraph satisfying $\Delta_{H} \simeq S^{|W|-2}$, with $W \neq \emptyset$. Then

$$
\Delta_{G} \simeq \Delta_{H} * \Delta_{G / W}
$$

Proof. By Theorem 14, we need merely prove $\bar{\Delta}_{G} \simeq \bar{\Delta}_{H} * \bar{\Delta}_{G / W}$.

Let $P=\mathcal{F}\left(\bar{\Delta}_{H} * \bar{\Delta}_{G / W}\right)$ and $Q=\mathcal{F}\left(\bar{\Delta}_{G}\right)$ be the associated face posets. Every $p$ in $P$ is a nonempty simplex of $\bar{\Delta}_{H} * \bar{\Delta}_{G / W}$, so we will simply write $X \cup Y$ for elements of $P$.

Define $f: P \rightarrow Q$ by

$$
f(X \bigcup Y)= \begin{cases}X \cup Y, & \text { if } \diamond \notin Y ; \\ W \cup Y \backslash\{\diamond\}, & \text { if } \diamond \in Y ;\end{cases}
$$

with $X \in \bar{\Delta}_{H}$ and $Y \in \bar{\Delta}_{G / W}$. Observe that $X \subseteq W$ and $Y \subseteq V \backslash W \cup\{\diamond\}$.

Recall: $\diamond$ is the state of $G / W$ that represents $W$ collapsed to a point.

[Comment: Observe the power of posets: $f$ maps a singleton set $\{\diamond\}$ to all of $W$.]

The Quillen Fiber Lemma will give us the desired result, if we can satisfy these preconditions:

(i) $f$ is well-defined, meaning that $f(X \cup Y)$ really is a simplex of $\bar{\Delta}_{G}$;

(ii) $f$ is order-preserving;

(iii) the fibers $f^{-1}\left(Q_{\leq q}\right)$ are contractible.

Establishing these three conditions is a bit tedious. There are several cases. We will prove the most interesting cases and leave the rest to the reader. We emphasize that one really must prove (i); full controllability within $H$ is needed to establish that $f$ is well-defined. 
(i). We assume $\diamond \in Y$. We leave to the reader the case in which $\diamond \notin Y$.

Let $X \in \bar{\Delta}_{H}$ and $Y \in \bar{\Delta}_{G / W}$ be given, with $\diamond \in Y$. This means there exists a stochastically acyclic set of actions $\tau^{\prime} \in \Delta_{G / W}$ such that $V_{\tau^{\prime}}=Y$. Now let $\tau$ be the actions of $G$ that generate $\tau^{\prime}$ (recall: the correspondence between actions in $G$ and $G / W$ is bijective). Since $\diamond \in Y, \tau^{\prime}$ includes an action $A^{\prime}$ with source $\diamond$. We may assume there is exactly one such action in $\tau^{\prime}$. Let $w_{0} \in W$ be the source of the corresponding action $A$ of $\tau$. Since $\Delta_{H} \simeq S^{|W|-2}$, the proof of Theorem 11 implies a stochastically acyclic set of actions $\sigma \in \Delta_{H}$ such that $V_{\sigma}=W \backslash\left\{w_{0}\right\}$. It is not hard to see that $\sigma \bigcup \tau$ is stochastically acyclic since $\tau^{\prime}$ is (Lemma 17(c) makes part of the argument, establishing that $\sigma \bigcup \tau \backslash\{A\}$ is stochastically acyclic). Finally,

$$
V_{\sigma} \bigcup \tau=W \backslash\left\{w_{0}\right\} \bigcup Y \backslash\{\diamond\} \bigcup\left\{w_{0}\right\}=W \bigcup Y \backslash\{\diamond\}
$$

meaning $f(X \cup Y) \in \bar{\Delta}_{G}$.

(ii). Easy.

(iii). Every $q \in Q$ is a nonempty simplex of $\bar{\Delta}_{G}$, so we will write $\bar{V}$ in place of $q$, with $\bar{V} \subset V$. Let $\bar{V}$ be given. We need to show that $f^{-1}\left(Q_{\leq \bar{V}}\right)$ is contractible.

We deal here with the case in which $\bar{V} \cap W=W$. We leave to the reader the case in which $\bar{V} \cap W$ is a proper subset of $W$.

To establish contractibility, we will show that $f^{-1}\left(Q_{\leq \bar{V}}\right)$ is the face poset of a cone with apex $\diamond$. Observe that $f^{-1}\left(Q_{\leq \bar{V}}\right)$ is indeed the face poset of a simplicial complex, since $f$ is order-preserving.

Let $X \bigcup Y \in f^{-1}\left(Q_{\leq \bar{V}}\right)$, with $X \in \bar{\Delta}_{H}$ and $Y \in \bar{\Delta}_{G / W}$. Suppose $\diamond \notin Y$. We need to show that $X \cup Y \cup\{\diamond\}$ is an element of $f^{-1}\left(Q_{\leq \bar{V}}\right)$. Observe:

$$
\begin{aligned}
& f(X \cup Y)=X \cup Y \subseteq \bar{V}, \quad \text { since } X \cup Y \in f^{-1}\left(Q_{\leq \bar{V}}\right) ; \\
& f(X \cup Y \cup\{\diamond\})=W \cup Y \subseteq \bar{V}, \quad \text { since } W \subseteq \bar{V} \text {. }
\end{aligned}
$$

There remains to show that $Y \bigcup\{\diamond\} \in \bar{\Delta}_{G / W}$. Suppose that is false. Then there must be some set of states $Y^{\prime} \subseteq Y$, such that every proper subset of $Y^{\prime} \bigcup\{\diamond\}$ is a simplex of $\bar{\Delta}_{G / W}$ but $Y^{\prime} \bigcup\{\diamond\}$ is not. By Lemma 15, no action of $G / W$ moves off $Y^{\prime} \bigcup\{\diamond\}$, which means no action of $G$ moves off $Y^{\prime} \cup W$. On the other hand, $Y^{\prime} \cup W \subseteq Y \cup W \subseteq \bar{V}$, implying $Y^{\prime} \cup W \in \bar{\Delta}_{G}$. Now Lemma 15 reveals a contradiction.

The same argument shows that $\{\diamond\}$ is itself in $f^{-1}\left(Q_{\leq \bar{V}}\right)$.

Theorem 20 (Controllability Structure) Let $G$ be a stochastic graph. Then

$$
\Delta_{G} \simeq S^{n-k-1} * \Delta_{G / \leftrightarrow},
$$

where $n$ is the size of $G$ 's state space and $k$ is the number of equivalence classes induced by $\leftrightarrow$.

Proof. Use Lemma 19 repeatedly on the equivalence classes determined by $\leftrightarrow$. Bear in mind that $S^{i} * S^{j} \simeq S^{i+j+1}[6]$.

Theorem 20 generalizes a result of Hultman's for directed graphs [44]. For directed graphs, it is not hard to see that $\Delta_{G / \leftrightarrow}$ is either the empty complex or homotopic to a point. So for directed graphs, $\Delta_{G}$ always looks like a sphere or a point, homotopically. For stochastic 
(in particular, nondeterministic) graphs, the domain is considerably richer (see Section 13 for instance); $\Delta_{G / \leftrightarrow}$ may have arbitrary homotopy type (with $n$ and $k$ varying) within the range of finite simplicial complexes.

We may think of $S^{n-k-1}$ as measuring controllability in the graph $G$, whereas $\Delta_{G / \leftrightarrow}$ captures the adversary's ability to constrain the system. The full meaning of the factor $\Delta_{G / \leftrightarrow}$ is still a research question. We learned a lot in Section 10; the following example offers further insight.

\subsection{An Example (Air Travel During Thunderstorm Season)}

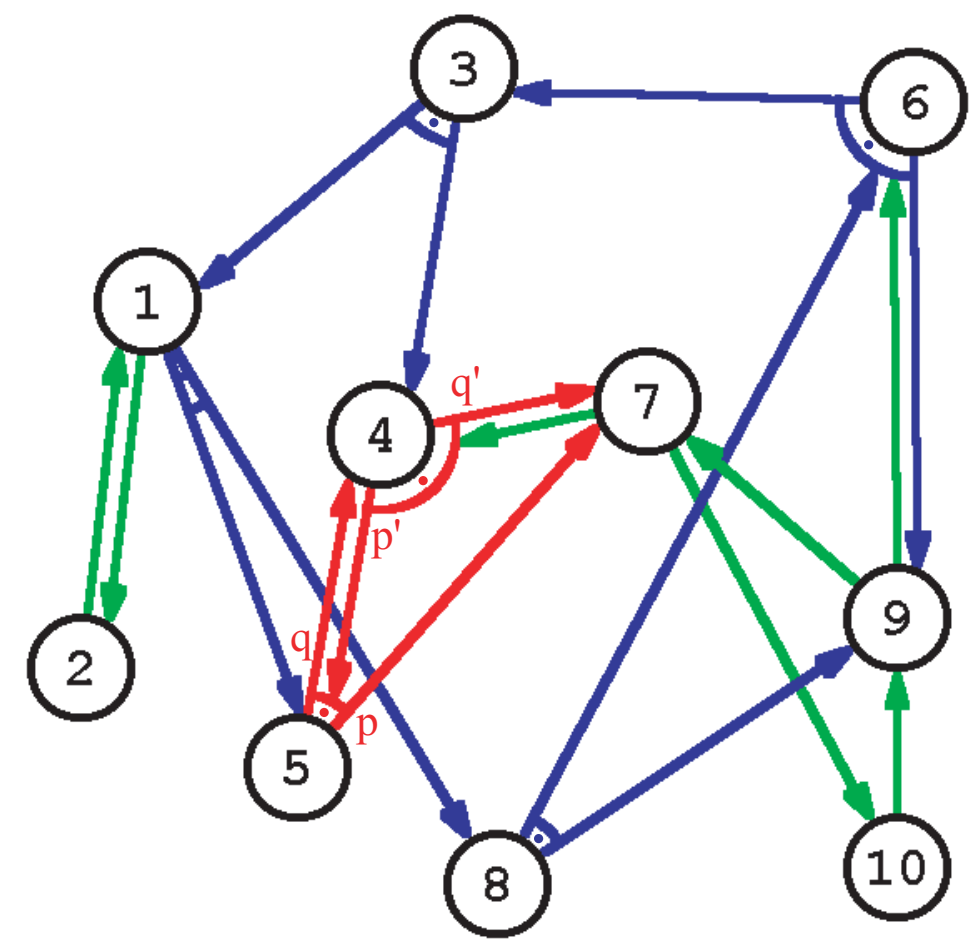

Figure 23: This graph contains three types of actions: deterministic (green), stochastic (red), and nondeterministic (blue). The graph might represent some poor passengers' potential flight paths during a day of thunderstorms.

Consider the graph of Fig. 23, which might represent possible air travel routes between cities in the United States during some thunderstorm-infested July afternoon. The graph includes deterministic, stochastic, and nondeterministic actions. Perhaps deterministic actions represent flights that are certainly possible, stochastic actions represent flights whose destinations are stochastically determined by emerging thunderstorms, and nondeterministic actions represent sets of possible flights, to any one of which a hopeful passenger will be assigned in an otherwise unpredictable fashion (nondeterminism is Murphy's Law in action).

Of interest is the set of cities reachable from anywhere in the system and whether a passenger might become trapped in an endless loop trying to reach some particular city.

The equivalence relation $\leftrightarrow$ partitions the state space into five equivalence classes. Three 


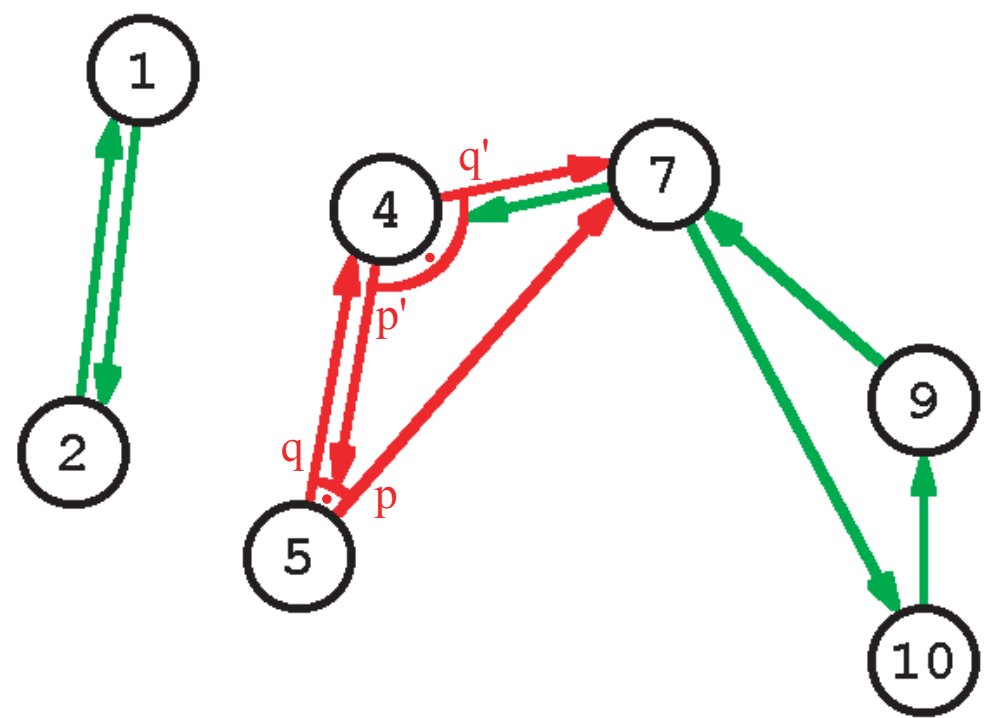

Figure 24: The two nontrivial equivalence classes of the $\leftrightarrow$ relation for the graph of Fig. 23, along with the actions establishing full controllability within each equivalence class.

of these are the singleton sets $\{3\},\{6\}$, and $\{8\}$. The two nontrivial equivalence classes are $\{1,2\}$ and $\{4,5,7,9,10\}$, as shown in Fig. 24 .

This means that passengers wishing to travel between cities 1 and 2 are in luck. They can go directly from one city to the next. Passengers traveling between cities in the set $\{4,5,7,9,10\}$ are also fairly lucky. They will certainly get to their destinations, though the exact routing may not always be certain in advance.

Unfortunately, there is a convention in city 4, towards which passengers from all over the country are traveling. Many will not make it today, as we shall see.

The left panel of Fig. 25 shows the quotient graph $G / \leftrightarrow$ for the graph $G$ in Fig. 23. Each equivalence class is represented by one of its states. The source complex $\bar{\Delta}_{G / \leftrightarrow}$ of this graph appears in Fig. 26. The underlying vertex set of $\bar{\Delta}_{G / \leftrightarrow}$ is the state space of $G / \leftrightarrow$, represented by the cities $\{1,3,4,6,8\}$. (Keep in mind that, for instance, state 4 really represents the entire equivalence class of cities $\{4,5,7,9,10\}$. Theorem 20 assures us that the capabilities of the graph overall are captured by this reduced quotient representation.)

The complements of the maximal simplices of $\bar{\Delta}_{G / \leftrightarrow}$ describe the most precisely attainable goals, as implied by the results of Section 10. Since $\{1,3,4,8\}$ is a simplex in $\bar{\Delta}_{G / \leftrightarrow}$, this means city 6 is attainable from anywhere within the graph $G / \leftrightarrow$ and thus from anywhere in the graph $G$. The right panel of Fig. 25 exhibits a strategy (in the quotient graph) for attaining city 6 . Too bad the convention is not happening in that city.

Particularly informative are the minimal nonfaces of $\bar{\Delta}_{G / \leftrightarrow}$. The complements of these sets are maximal simplices in $\bar{\Delta}_{G / \leftrightarrow}^{*}$, meaning they are the maximal goals not certain to be attainable from everywhere in the graph $G / \leftrightarrow \cdot \bar{\Delta}_{G / \leftrightarrow}$ contains two minimal nonfaces, namely $\{4,6\}$ and $\{1,3,6,8\}$. The complement of $\{1,3,6,8\}$ with respect to the the underlying vertex set of $\bar{\Delta}_{G / \leftrightarrow}$ is $\{4\}$. Consequently, city 4 , the location of the convention, is not stochastically attainable from everywhere in the system. 

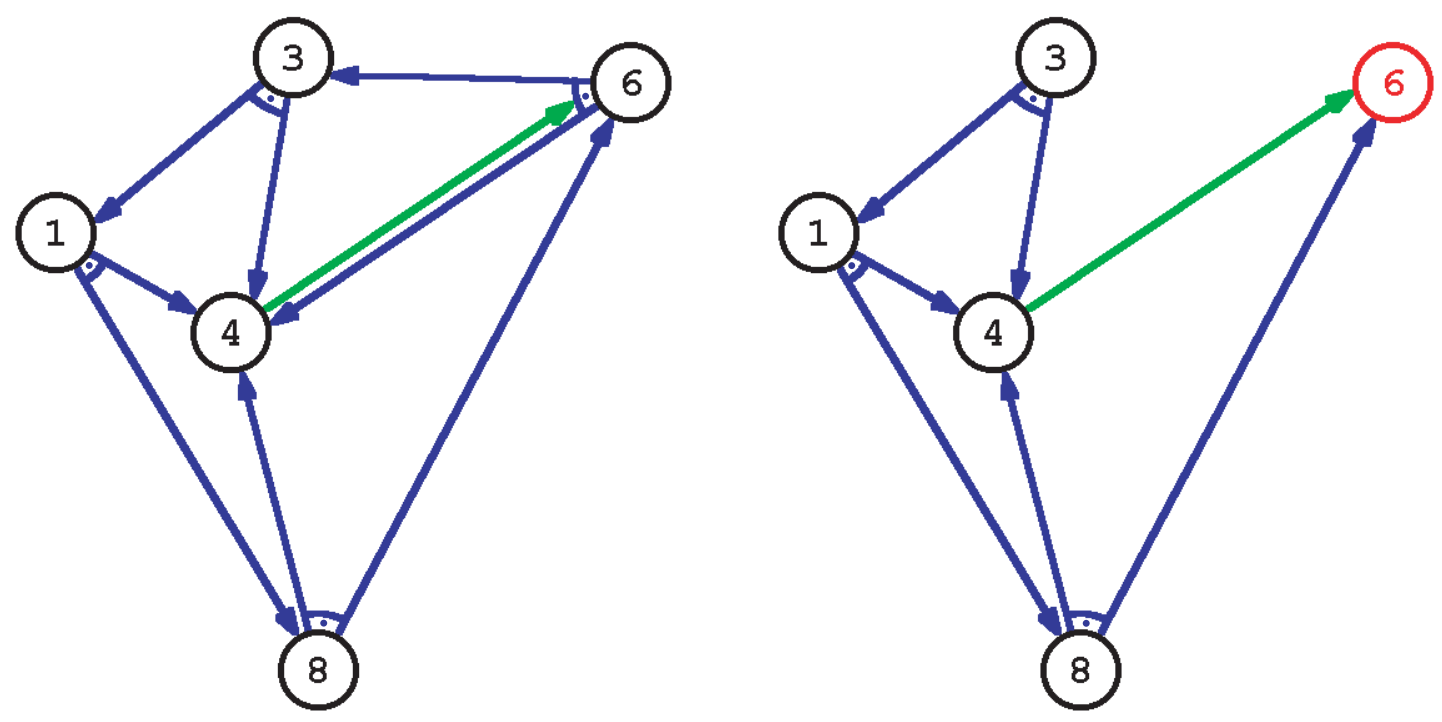

Figure 25: The left panel shows the quotient graph $G / \leftrightarrow$ obtained by collapsing the strongly controllable subspaces of the graph $G$ in Fig. 23 to single states (not shown are self-looping actions). The right panel shows a particular strategy for attaining state 6 in this quotient graph.

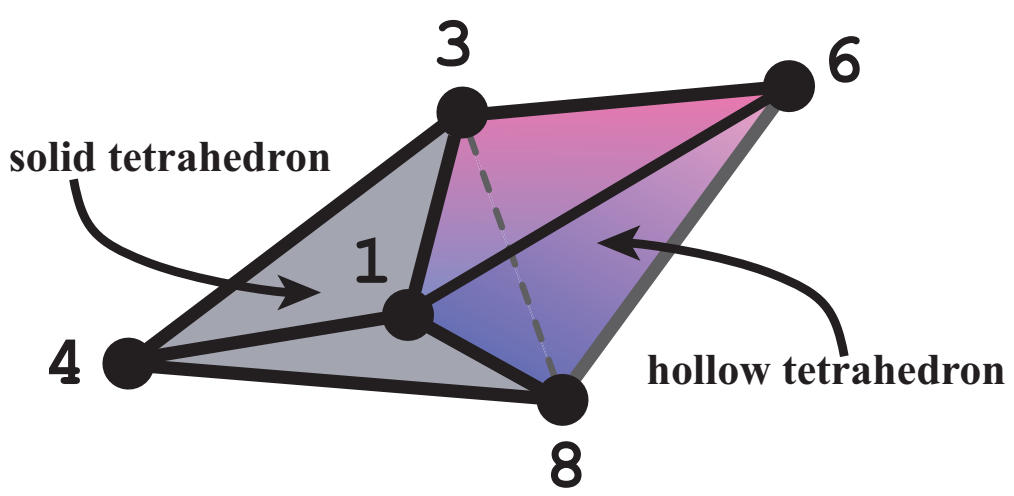

Figure 26: Source complex $\bar{\Delta}_{G / \leftrightarrow}$ of the quotient graph $G / \leftrightarrow$ of Fig. 25 . 

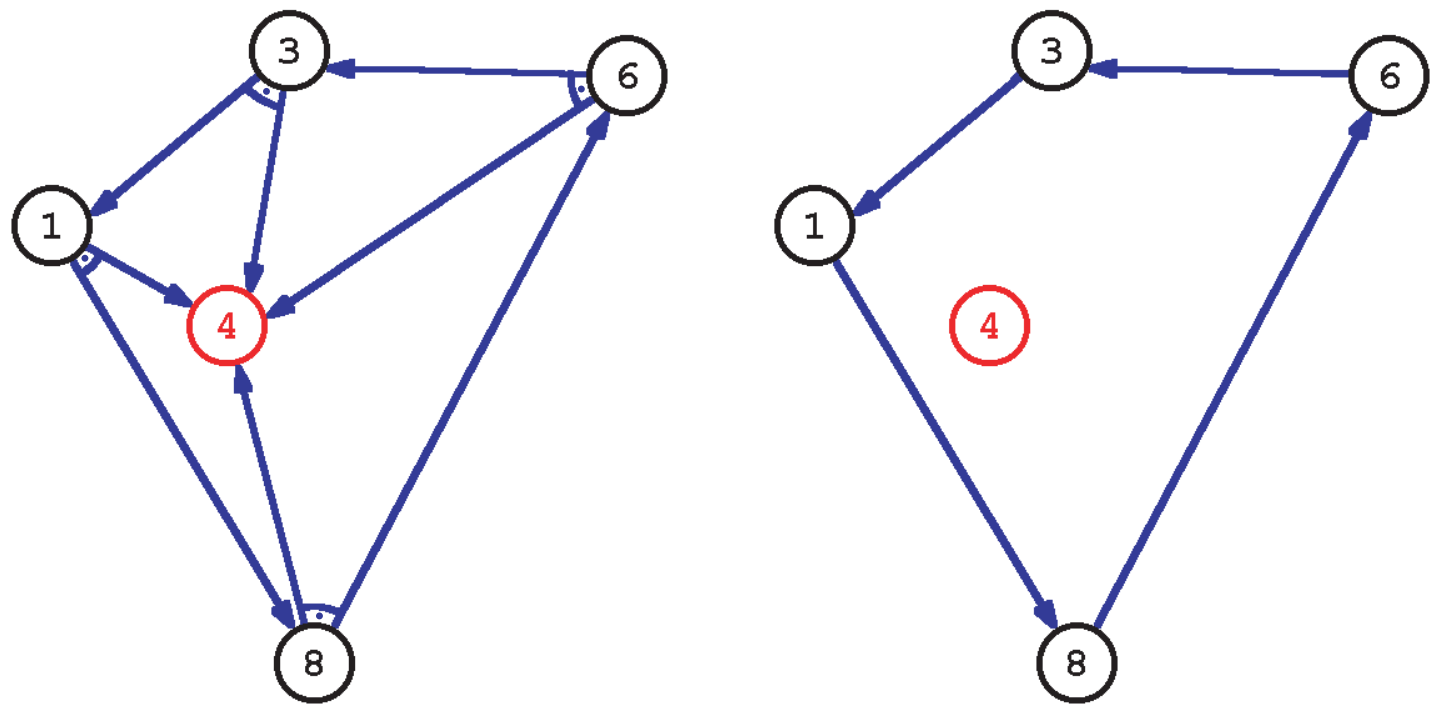

Figure 27: The graph $(G / \leftrightarrow) \mid W$ appears in the left panel, with $W=\{1,3,6,8\}$ being a minimal nonface of $\bar{\Delta}_{G / \leftrightarrow}$ (see Fig. 26). An adversary can prevent attainment of state 4 by selecting transitions as in the right panel. The system then has full controllability within $W$ relative to this adversarial choice.

The left panel of Fig. 27 shows the induced subgraph formed by flights available at the equivalence classes of cities appearing in the minimal nonface $\{1,3,6,8\}$. Sure enough, adversarially, city 4 may become unreachable, as the right panel of Fig. 27 demonstrates.

Remark: The reader may have observed that in the right graph of Fig. 27, although city 4 becomes unreachable, the system can move to any city within the minimal nonface $\{1,3,6,8\}$. This is in fact generally true: if some set $W$ is a nonempty minimal nonface of $\bar{\Delta}_{H}$ for some graph $H$, then the system has full controllability within $W$ relative to the assumption that it does not exit from $W$. In other words, it might happen that the system leaves $W$, but if not, for instance if an adversary makes nondeterministic choices to prevent motions out of $W$, then the system can move to any state within $W$ from any other state in $W$. This is consistent with the observation we made in the proof of Theorem 16 that $\bar{\Delta}_{G \mid W} \simeq S^{|W|-2}$ for any such $W$.

\section{Algorithms}

This section provides algorithms for backchaining from a goal set and for computing a graph's strongly controllable subspaces. With these tools one can then reduce a graph to its potentially simpler quotient graph, as well as compute the source complex of the graph or of its quotient graph. One then obtains a characterization of the underlying system's capabilities, as discussed in the previous sections. We have implemented all these algorithms. Sample runs produced the figures in Section 11.4.

We do not provide an explicit algorithm for computing the strategy complex of a graph. Our homotopy results show that one does not need the strategy complex per se in order 
to understand a system's capabilities. Moreover, the algorithm BACKCHAIN given next will produce a strategy for attaining any particular goal set $S$, or determine that no strategy exists. However, there certainly may exist strategies in a strategy complex other than those computed by backchaining, as Fig. 5 suggests. These extra strategies may be useful practically as backup strategies. The procedure outlined in Section 11.2, particularly starting with "Now suppose ..." on page 48, allows one to construct such strategies individually or as part of the full strategy complex.

\section{Algorithm $1 \quad \operatorname{BaCKChain}(G, S)$}

Input: $\quad$ A stochastic graph $G=(V, \mathcal{A})$ and a nonempty subset $S$ of $V$.

Output: A (possibly empty) set $\left\{v_{1}, \ldots, v_{k}\right\}$ of states in $V \backslash S$ and a corresponding set $\left\{\sigma_{1}, \ldots, \sigma_{k}\right\}$ of collections of actions in $\mathcal{A}$, such that every action in $\sigma_{i}$ has source $v_{i}$ and moves off the set $\left\{v_{i}, \ldots, v_{k}\right\}$, for $i=1, \ldots, k$. Moreover, if $v$ is any state in $V \backslash S$ for which $G$ contains a stochastic strategy for attaining $S$ from $v$, then $v$ will be one of the states $v_{i}$ returned.

\section{Procedure:}

1. Let $W_{0}:=S$.

2. For $i=1,2, \ldots$ until done do:

(a) Let $v_{i}$ be any state in $V \backslash W_{i-1}$ such that some action with source $v_{i}$ moves off $V \backslash W_{i-1}$. If no such $v_{i}$ exists, then done.

(b) Otherwise, let $\sigma_{i}$ be all actions in $\mathcal{A}$ with source $v_{i}$ that move off $V \backslash W_{i-1}$.

(c) Let $W_{i}:=W_{i-1} \bigcup\left\{v_{i}\right\}$.

3. Return $\left\{v_{1}, \ldots, v_{k}\right\}$ and $\left\{\sigma_{1}, \ldots, \sigma_{k}\right\}$, with $k$ the maximum index for which $v_{i}$ is defined. (If there is no such $k$, then return $\emptyset$ and $\emptyset$.)

Remarks: When Algorithm 1 is done, the collection of actions $\sigma=\bigcup_{i=1}^{k} \sigma_{i}$ is stochastically acyclic, constituting a strategy for attaining $V \backslash\left\{v_{1}, \ldots, v_{k}\right\}$. In particular, if $W_{k}=V$ when Step 2 is done, then $\sigma$ is a complete stochastic strategy for attaining $S$ on the state space $V$. Conversely, if $S$ is stochastically attainable in $G$, then $W_{k}$ will be all of $V$, as the proof of Theorem 16 shows.

Aside: Applying Algorithm 1 to the graph of Fig. 5 with $S=\{3\}$ returns one or the other of the two triangles in the strategy complex shown in the figure, rather than merely the central edge. This is because Algorithm 1 grows the sets $W_{i}$ one state at a time. Traditionally, one might add multiple states at once. Doing so would then produce the central edge as the backchained strategy in Fig. 5. 
Given a goal $S$, the next algorithm uses backchaining to iteratively winnow a graph's state space $V$ down to the maximal subspace $W$ within which $S$ is stochastically attainable. This procedure will be a useful step in determining a graph's strongly controllable subspaces.

(The reader may wish to verify that the is correct, that there really is a single maximal subspace $W$ within which $S$ is stochastically attainable.)

\section{Algorithm $2 \quad$ Strategy $(G, S)$}

Input: $\quad$ A stochastic graph $G=(V, \mathcal{A})$ and a nonempty subset $S$ of $V$.

Output: A stochastically acyclic subgraph $H=(W, \mathcal{B})$ of $G$ such that $S \subseteq W$ and $V_{\mathcal{B}}=W \backslash S$, with $W$ maximal among all such $W$.

\section{Procedure:}

1. Let $\left\{v_{1}, \ldots, v_{k}\right\},\left\{\sigma_{1}, \ldots, \sigma_{k}\right\}$ be the results returned by $\operatorname{BaCkchain}(G, S)$.

2. Let $W:=\left\{v_{1}, \ldots, v_{k}\right\} \bigcup S$ and $\sigma:=\bigcup_{i=1}^{k} \sigma_{i}$.

3. If $W=V$, return the graph $(W, \sigma)$.

4. Otherwise, recursively call $\operatorname{Strategy}((W, \mathcal{C}), S)$, where $\mathcal{C}$ consists of all actions in $\mathcal{A}$ whose sources and targets lie in W.

Given a stochastic graph $G=(V, \mathcal{A})$, the next algorithm computes a regular directed graph $(V, E)$ such that $(v, u)$ is an edge in $E$ if and only if $G$ contains a stochastic strategy for attaining $u$ from $v$, with $v$ distinct from $u$. (See again the definitions on page 31.)

\section{Algorithm $3 \quad$ Reachable $(G)$}

Input: $\quad$ A stochastic graph $G=(V, \mathcal{A})$, with $V \neq \emptyset$.

Output: A directed graph $D=(V, E)$, with specifications as above.

\section{Procedure:}

1. For each $u \in V$, let $H_{u}:=\left(W_{u}, \mathcal{B}_{u}\right)$ be the result returned by $\operatorname{Strategy}(G,\{u\})$.

2. Let $E:=\left\{(v, u) \in V \times V \mid v \in W_{u}, v \neq u\right\}$.

3. Return $(V, E)$. 
The next algorithm computes the strongly controllable subspaces of $G$.

\section{Algorithm $4 \quad \operatorname{Subspaces}(G)$}

Input: $\quad$ A stochastic graph $G=(V, \mathcal{A})$, with $V \neq \emptyset$.

Output: The set $\left\{W_{1}, \ldots, W_{m}\right\}$ of equivalence classes of $\leftrightarrow$ for $G$.

\section{Procedure:}

1. Let $\left\{V_{1}, \ldots, V_{\ell}\right\}$ be the strongly connected components of the directed graph returned by $\operatorname{Reachable}(G)$. See [1] for an algorithm to compute these components.

2. If $\ell=1$, return $\left\{V_{1}\right\}$.

3. Otherwise, for each $i=1, \ldots, \ell$ :

(a) Let $H_{i}:=\left(V_{i}, \mathcal{C}_{i}\right)$, with $\mathcal{C}_{i}$ all actions of $\mathcal{A}$ whose sources and targets lie in $V_{i}$.

(b) Let $\mathcal{V}_{i}$ be the result of calling $\operatorname{SubspaCES}\left(H_{i}\right)$ recursively.

$\operatorname{Return} \bigcup_{i=1}^{\ell} \mathcal{V}_{i}$

\section{Remarks:}

- Correctness. It is not difficult to prove that the previous algorithms correctly compute output as specified.

- Runtime. The previous algorithms all run in almost-reasonable polynomial time. For instance, the slowest, $\operatorname{Subspaces}(G)$, has time-complexity $O\left(|V|^{5}|\mathcal{A}|\right)$, with $G=(V, \mathcal{A})$. No doubt, faster implementations exist.

- Quotient Graphs. Computing the quotient graph $G / \leftrightarrow$ from a graph $G$ entails calling $\operatorname{Subspaces}(G)$, then relabeling sources and targets of actions. Specifically, if $\operatorname{Subspaces}(G)$ returns $\left\{W_{1}, \ldots, W_{m}\right\}$, then one relabels every state $w$ in $W_{i}$ by some representative state for $W_{i}$, with $i=1, \ldots, m$.

- Source Complexes. Computing the source complex $\bar{\Delta}_{G}$ of a graph $G$ is conceptually straightforward: For every possible goal set $S$, one calls BackChain $(G, S)$. To see this:

As we observed earlier, if the set of states $\left\{v_{1}, \ldots, v_{k}\right\}$ returned by $\operatorname{Backchain}(G, S)$ is equal to $V \backslash S$, then $S$ is a stochastically attainable goal. Always, $\left\{v_{1}, \ldots, v_{k}\right\}$ is a simplex of $\bar{\Delta}_{G}$. Conversely, any simplex of $\bar{\Delta}_{G}$ must show up as the start region of some minimal complete stochastic strategy for attaining some goal $S$ in $G$. By itself, without other actions of $G$, that strategy necessarily looks like a backchained strategy, as can be seen via recursive application of Lemma 15. Consequently, given other actions of $G$, backchaining may produce a different strategy, but it will produce some complete stochastic strategy for attaining $S$ in $G$.

This naïve algorithm has running time exponential in the size of $V$. One can tinker with the procedure to make it slightly more efficient, but the exponential nature is likely to be fundamental, as the first hardness result of Section 14 indicates. 


\section{Realizability}

We have previously indicated that finite simplicial complexes and stochastic graphs are essentially equivalent objects from a topological perspective. In fact, this equivalence depends on nondeterminism not stochasticity. The underlying reason is that finite simplicial complexes are purely combinatorial and thus are equivalent to finite posets $[6,75]$. Nondeterministic graphs alone, without stochastic actions, are able to capture all finite posets.

This section makes precise these comments with two realizability theorems. The first theorem says that for any finite simplicial complex $\Sigma$, there is some strategy complex isomorphic to the first barycentric subdivision of $\Sigma$. The second theorem says that $\Sigma$ may actually be realized exactly as a source complex of some graph. In both cases, the generating graphs are nondeterministic.

Reminder: $\operatorname{sd}(\Sigma)$ means the first barycentric subdivision of $\Sigma$ (Section 5.4).

Theorem 21 (Realization by Strategy Complexes) Let $\Sigma$ be a finite simplicial complex. There exists a nondeterministic graph $G$ such that $\operatorname{sd}(\Sigma) \cong \Delta_{G}$.

Proof. If $\Sigma=\emptyset$, then $\operatorname{sd}(\Sigma)=\emptyset$, so we may let $G=(\emptyset, \emptyset)$.

Otherwise, define $G=(V, \mathcal{A})$ as follows: $V$ consists of all vertices in the complex $\operatorname{sd}(\Sigma)$ along with one new vertex, $\perp$. $\mathcal{A}$ contains exactly one nondeterministic action $A_{v}$ at every $v \in V$ other than at $\perp . A_{v}=\{(v, u) \mid u \in V$ and $\{v, u\}$ is not a simplex of $\operatorname{sd}(\Sigma)\}$. In other words, $A_{v}$ has transitions to all vertices that are not adjacent or equal to $v$ in the barycentric subdivision of $\Sigma$. Observe that every action $A_{v}$ contains at least one edge, namely a transition from $v$ to $\perp$, so this definition is well-formed. Moreover, no action contains a self-loop.

The map $v \mapsto A_{v}$ is a bijective correspondence between the vertices of $\operatorname{sd}(\Sigma)$ and those of $\Delta_{G}$. In order to establish that $\operatorname{sd}(\Sigma) \cong \Delta_{G}$, we need to show that this correspondence preserves simplices. Definitionally, both $\operatorname{sd}(\Sigma)$ and $\Delta_{G}$ contain the empty simplex.

(Aside: If $\Sigma=\{\emptyset\}$, then $G=(\{\perp\}, \emptyset)$ and $\Delta_{G}=\{\emptyset\}=\operatorname{sd}(\Sigma)$.)

(a) Suppose $\emptyset \neq \sigma \in \operatorname{sd}(\Sigma) . \quad \sigma=\left\{v_{1}, \ldots, v_{k}\right\}$, with $v_{i} \in V \backslash\{\perp\}$. Let $A_{i}$ be the action of $G$ defined at $v_{i}$, for $i=1, \ldots, k$. No $v_{i}$ can appear as the target of any $A_{j}$, since $\left\{v_{i}, v_{j}\right\}$ is a simplex of $\operatorname{sd}(\Sigma)$. That means $\left\{A_{1}, \ldots, A_{k}\right\}$ is a simplex of $\Delta_{G}$.

(b) Suppose $\emptyset \neq \tau \in \Delta_{G} . \quad \tau=\left\{A_{1}, \ldots, A_{k}\right\}$, with each $A_{i} \in \mathcal{A}$. Let $v_{i}$ be the source of $A_{i}$, for $i=1, \ldots, k$. Each $v_{i}$ is the barycenter of some nonempty simplex $\sigma_{i} \in \Sigma$.

Consider the set $\left\{v_{i}, v_{j}\right\}$ formed by any two distinct such vertices. This set must be a simplex of $\operatorname{sd}(\Sigma)$ as otherwise $\left\{A_{i}, A_{j}\right\}$ could generate a cycle in $G$. Consequently, either $\sigma_{i}$ is a proper face of $\sigma_{j}$ or vice-versa. So, without loss of generality, $\emptyset \subset \sigma_{1} \subset \sigma_{2} \subset \cdots \subset \sigma_{k}$, all inclusions being proper. In turn, that means $\left\{v_{1}, \ldots, v_{k}\right\}$ is a $\operatorname{simplex}$ of $\operatorname{sd}(\Sigma)$.

Theorem 22 (Realization as Source Complexes) Let $\Sigma$ be a finite simplicial complex. There exists a nondeterministic graph $G$ such that $\Sigma=\bar{\Delta}_{G}$. 
Proof. If $\Sigma=\emptyset$, let $G=(\emptyset, \emptyset)$. Then $\bar{\Delta}_{G}=\emptyset$.

Otherwise, let $V$ be the vertices of $\Sigma$. If $\Sigma$ is the complex of the full simplex on $V$, enlarge $V$ by one new state, $\perp$. Let $\bar{\Sigma}$ be the set of maximal simplices of $\Sigma$. Observe: By construction of $V, V \notin \bar{\Sigma}$.

Construct $G=(V, \mathcal{A})$ as follows: For every $X \in \bar{\Sigma}$ and every $v \in X$, define the nondeterministic action $A_{v, X}=\{(v, u) \mid u \in V \backslash X\}$. Each such action contains at least one edge, since $X$ cannot be all of $V$. Let $\mathcal{A}$ consist of all such actions.

(Aside: If $\Sigma=\{\emptyset\}$, then $G=(\{\perp\}, \emptyset)$ and $\bar{\Delta}_{G}=\{\emptyset\}$.)

(a) Suppose $\emptyset \neq X \in \Sigma$. We can assume without loss of generality that $X \in \bar{\Sigma}$. Consider the set of actions $\sigma=\left\{A_{v, X} \mid v \in X\right\}$. No source of an action in $\sigma$ is the target of an action in $\sigma$, so $\sigma \in \Delta_{G}$. Thus $X=V_{\sigma} \in \bar{\Delta}_{G}$.

(b) Suppose $\emptyset \neq X \in \bar{\Delta}_{G}$. Let $\sigma \in \Delta_{G}$ such that $V_{\sigma}=X$. By Lemma $15, \sigma$ contains an action that moves off $X$. The action must be of the form $A_{v, Y}$, for some $Y \in \bar{\Sigma}$, with $v \in Y$. By the definition of "moves off", $v \in X$ and all targets of $A_{v, Y}$ lie outside $X$. By construction, the targets of $A_{v, Y}$ are all the states of $V \backslash Y$. That means $X \subseteq Y$. So $X$ is a simplex of $\Sigma$, since $Y$ is.

Both $\Sigma$ and $\bar{\Delta}_{G}$ contain the empty simplex.

Remark: The underlying vertex sets of $\Sigma$ and $\bar{\Delta}_{G}$ may differ slightly, but the simplices in the two complexes are identical.

\section{Hardness}

This section presents some simple hardness results. The first result indicates that the exponential computation of $\bar{\Delta}_{G}$ described in Section 12 is fundamental, assuming $N P$ and $P$ are different complexity classes. The other two results suggest that homotopy equivalence of strategy complexes is either a stronger topological condition than one needs for understanding system capabilities or that homotopy equivalence encodes some interesting properties about uncertain systems beyond those one usually asks at the fixed graph level. It remains a research question to know which of these perspectives is correct.

\subsection{The Difficulty of Determining a System's Precision}

The first result has practical implications. Given an uncertain system, it may be very difficult to determine just how precise the system can be.

Lemma 23 (Precision is Hard) Let $G=(V, \mathcal{A})$ be a stochastic graph. Determining the size of the smallest stochastically attainable goal is NP-complete. 
Proof. The proof is by a reduction from Independent Set. The underlying ideas are related to the realizability ideas from Section 13.

We define the following two problems:

InDEPSET: Given an undirected graph $(V, E)$ and an integer $\ell$, is there a set $I \subseteq V$ of size $\ell$ such that no two states in $I$ constitute an edge in $E$ ?

Precision: Given a stochastic graph $G=(V, \mathcal{A})$ and an integer $k$, is there some set $S \subseteq V$ of size $k$ such that $G$ contains a complete stochastic strategy for attaining $S$ ?

INDEPSET is a known $N P$-complete problem $[49,66]$.

I. Precision lies in NP:

Given $S$, one can verify in polynomial time that $S$ has size $k$ and that $G$ contains a complete stochastic strategy for attaining $S$, using Algorithm 1.

II. Precision is NP-hard:

Suppose $(V, E)$ is an undirected graph and $\ell$ an integer. Let $n=|V|$.

Define a nondeterministic graph $G=(V \cup\{\perp\}, \mathcal{A})$ as follows: $\perp$ is a new state. For every $v \in V, \mathcal{A}$ contains a nondeterministic action $A_{v}=\{(v, u) \mid u=\perp$ or $(v, u)$ is an edge in $E\}$. So $A_{v}$ transitions to every state adjacent to $v$ in the original undirected graph, as well as to $\perp$ (ensuring $A_{v}$ is well-formed).

(a) Suppose $I$ is an independent set of size $\ell$ in $(V, E)$.

Consider the set of actions $\sigma=\left\{A_{i} \mid i \in I\right\}$. No source of an action in $\sigma$ is the target of an action in $\sigma$, by definition of $I$. So $\sigma \in \Delta_{G}$. That means $G$ contains a complete strategy for attaining $\{\perp\} \cup V \backslash I$, which is a set of size $n+1-\ell$.

(b) Suppose $G$ contains a complete strategy for attaining set $S$ of size $k$.

$S$ necessarily contains $\perp$. There is some $\sigma \in \Delta_{G}$ such that $V_{\sigma}=V \backslash S$ and $\left|V_{\sigma}\right|=n+1-k$. $V_{\sigma}$ is an independent set in $(V, E)$. To see this, suppose otherwise. Then $\sigma$ must contain actions $A_{v}$ and $A_{u}$ such that $(v, u)$ is an edge in $E$. That would mean $\sigma$ could generate a cycle in $G$, a contradiction.

To summarize: $(V, E)$ contains an independent set of size $\ell$ if and only if $G$ contains a stochastically attainable goal of size $k$ with $k+\ell=n+1$. One may construct $G$ from $(V, E)$ in polynomial time. Thus PRECISION is NP-hard.

\subsection{Small Realization is Uncomputable}

There are classic deep results telling us it is algorithmically impossible to recognize the homeomorphism or homotopy type of even a finite general simplicial complex [76]. These results have their roots in work on the algorithmic impossibility of deciding whether a word in a finitely presented group is trivial [63]. One particular implication is that it is algorithmically uncomputable to decide whether a finite simplicial complex is contractible (this follows from 
[64]). We present two consequences for nondeterministic (and thus stochastic) graphs.

The following lemma says: Finding the smallest graph whose strategy complex is homotopic to a given complex is an uncomputable problem. Compare this with the realizability results of Section 13.

Lemma 24 (Compact Realization) The following question is undecidable:

Given a finite simplicial complex $\Sigma$ and a nonnegative integer $m$, is there a stochastic graph $G$ with $m$ actions such that $\Delta_{G} \simeq \Sigma$ ?

Proof. Observe: If $G$ is a stochastic graph containing a single action $A$, then either $\Delta_{G}=\{\emptyset\}$ or $\Delta_{G}=\{\emptyset,\{A\}\}$, depending on whether $A$ contains a (non)deterministic selfloop or not, respectively.

We now reduce from the uncomputability of deciding contractibility:

Suppose $\Sigma$ is a finite simplicial complex. We can check whether it is trivial (void or empty) easily. Assuming it is not trivial, we ask whether there is a stochastic graph $G$ containing a single action such that $\Delta_{G} \simeq \Sigma$. The answer is "yes" if and only if $\Sigma$ is contractible.

\subsection{Recognizing Repercussions is Uncomputable}

Suppose we are given a stochastic graph $G=(V, \mathcal{A})$ that somehow does not satisfy some criterion we desire. We wish to understand whether adding a particular new action $A$, with source and targets in $V$, will satisfy our criterion. We can certainly compute the strategy or source complexes of the resulting graph, to see whether any larger start regions or more precise goals have been created. What we cannot do, in general, is decide whether the homotopy type of the strategy (or source) complex has changed, as the following lemma indicates.

Lemma 25 (Detecting Change) The following question is undecidable:

Given a stochastic graph and a new action, does the graph's strategy complex change homotopy type when one adds the action to the graph?

Proof. Suppose $\Sigma$ is a finite simplicial complex. Again, we can check whether it is trivial (void or empty) easily. Assuming it is not trivial, we construct a graph $G$ much as in the proof of Theorem 22, except that we always add two new states, $\perp$ and $T$. So the graph looks like $G=(V, \mathcal{A})$, with $V$ the union of $\{\perp, \top\}$ and the vertices of $\Sigma$. The set of actions $\mathcal{A}$ is computed as in the proof of Theorem 22. Observe that there are no actions at either $\perp$ or $\top$. As before, one obtains exact equality: $\Sigma=\bar{\Delta}_{G}$.

Now consider adding to $G$ a new action $A$, representing a deterministic transition from $\top$ to $\perp$. Call the resulting graph $\widehat{G}$. Then $\Delta_{\widehat{G}}$ is a cone with apex $A$, hence contractible.

In summary:

- $\Sigma=\bar{\Delta}_{G} \simeq \Delta_{G}$ (by proof of Theorem 22 and by Theorem 14).

- $\Delta_{G} \simeq \Delta_{\widehat{G}}$ if and only if $\Delta_{G}$ is contractible (by construction).

So $\Sigma$ is contractible if and only if $\Delta_{G} \simeq \Delta_{\widehat{G}}$.

Recognizing contractibility is undecidable. 


\section{Topological Thinking}

This section discusses some scenarios in which topological thinking reveals the essence of a problem and its solution.

\subsection{Topology Precompiles an Existence Argument}

Suppose $G=(V, \mathcal{A})$ is a stochastic graph with associated nonnegative action transition times $\left\{\delta_{A}\right\}_{A \in \mathcal{A}}$. Let $S$ be a desired stop set (goal states) within $V$ and let $T$ be some nonnegative time.

Suppose for every $u$ in $V \backslash S$, someone has produced a strategy $\sigma_{u}$ by which the system will converge to $S$ when started at $u$, with worst-case expected-convergence time for $u$ no greater than $T$. (In other words, $0 \leq t_{u} \leq T$ in the solution of System (1) on p. 22, written out for $\sigma_{u}$. We make no assertions concerning $\sigma_{u}$ 's times $t_{v}$ for states $v$ other than $u$.)

We ask the question: Is there a complete stochastic strategy $\sigma$ that converges to $S$ from all states of $V$ with all worst-case expected-convergence times no greater than $T$ ?

Intuitively, we certainly expect the answer to be "yes". After all, one can imagine a metastrategy that determines the initial state $u$, then executes strategy $\sigma_{u}$. However, this dispatchbased strategy is not a strategy in our sense. Our strategies have the flavor of feedback-based control laws, that is, they are mappings from states to (sets of) actions. The question therefore is whether we can flatten the dispatch-based strategy to be a true strategy. Observe the difficulties:

Let $v$ and $u$ be distinct states in $V \backslash S$.

Issue 1: It could very well be that $\sigma_{u}$ contains no actions at state $v$.

Issue 2: Even if $\sigma_{u}$ contains one or more actions at state $v$, it could be that $t_{v}>T$ when we solve System (1) written out for $\sigma_{u}$.

Issue 3: The two strategies $\sigma_{u}$ and $\sigma_{v}$ may contain inconsistent actions, meaning that $\sigma_{u} \bigcup \sigma_{v}$ might not be stochastically acyclic. For instance, one strategy might move left through a hallway and the other right.

One can make a backchaining argument that some strategy $\sigma$ exists for attaining $S$ from everywhere in $V$. Arguing that among all such strategies there is at least one for which all worst-case expected convergence times are bounded by $T$ is possible, but tedious. 
There is a short topological argument:

- Write $V=[n]$ and $S=\{n\}$, without loss of generality.

- Consider the loopback graph $G_{\leftarrow n}=\left(V, \mathcal{A}^{\prime}\right)$, as in the proof of Theorem 1 . Associate transition time 0 to every loopback action.

- By Lemma 10, the affine cover $\bigcup_{A \in \mathcal{A}^{\prime}} U_{A}^{+}$contains all sets $\left\{\mathbf{x} \in \mathbf{R}^{n} \mid x_{i}>x_{n}+T\right\}$ and all sets $\left\{\mathbf{x} \in \mathbf{R}^{n} \mid x_{n}>x_{j}\right\}$.

Thus it contains all sets $\left\{\mathbf{x} \in \mathbf{R}^{n} \mid x_{i}>x_{j}+T\right\}$, with $i, j$ in $V$.

Using Theorem 8 and the structure of $\partial C_{T+\epsilon}$,

$$
\Delta_{G \leftarrow n}^{T} \simeq \bigcup_{A \in \mathcal{A}^{\prime}} U_{A}^{+} \bigcap \partial C_{T+\epsilon} \simeq S^{n-2}
$$

- By the remark at the end of Section 8 (p. 35), $\Delta_{G}^{T}$ contains a complete stochastic strategy for attaining $S$. In particular, all worst-case expected convergence times of this strategy are bounded by $T$, as desired.

The intuitive summary:

- Strategy existence depends on cover connectivity in $\mathbf{R}^{n}$.

- Adding strategies, even partial or pairwise inconsistent strategies, can only improve cover connectivity.

Topology has precompiled an existence argument: Bellman's Principle of Optimality shows up as the union of open sets.

\subsection{Topological Analysis of Adversity}

In looking at examples, the reader may have begun to wonder where adversity sits: In the stochastic nature of actions or in the nondeterministic nature of actions? Topology helps us understand an answer to that question.

The definition of moves off on page 41 makes explicit a difference between stochastic and nondeterministic actions: A stochastic action is almost the same as a set of deterministic actions. The following definition and lemma make this idea precise:

Definition: If $G=(V, \mathcal{A})$ is a stochastic graph, let $\operatorname{det}(G)=(V, \operatorname{det}(\mathcal{A}))$ designate the nondeterministic graph in which every stochastic action of $G$ has been replaced by a set of deterministic actions. Specifically, let us $\operatorname{define} \operatorname{det}(\mathcal{A})$ as follows:

- $\operatorname{det}(\mathcal{A})$ contains every deterministic or nondeterministic action of $\mathcal{A}$.

- For every stochastic action $A \in \mathcal{A}$ of the form $A=\left\{\left(v, p_{1} u_{1}\right), \ldots,\left(v, p_{k} u_{k}\right)\right\}, \operatorname{det}(\mathcal{A})$ contains $k$ deterministic actions, each consisting of a single edge $\left(v, u_{i}\right)$, with $i=1, \ldots, k$. (As usual, we permit redundancies, that is, distinct actions with identical edge sets.) 
Lemma 26 (Stochastic Determinism) Let $G=(V, \mathcal{A})$ be a stochastic graph. Then

$$
\Delta_{G} \simeq \Delta_{\operatorname{det}(G)} \quad \text { and } \quad \bar{\Delta}_{G}=\bar{\Delta}_{\operatorname{det}(G)} .
$$

Proof. The first assertion follows from the second, by Theorem 14 .

One can establish the second assertion by using Algorithm 1 and Lemma 15.

Conclusion: From the perspective of homotopy type or from the perspective of understanding a system's stochastically attainable goals, one may study $\operatorname{det}(G)$ in place of $G$. Fig. 28 provides an example, demonstrating homotopy equivalence of $\Delta_{G}$ and $\Delta_{\operatorname{det}(G)}$. Of course, convergence times in the two graphs may very well be different, so one must be careful not to oversimplify.
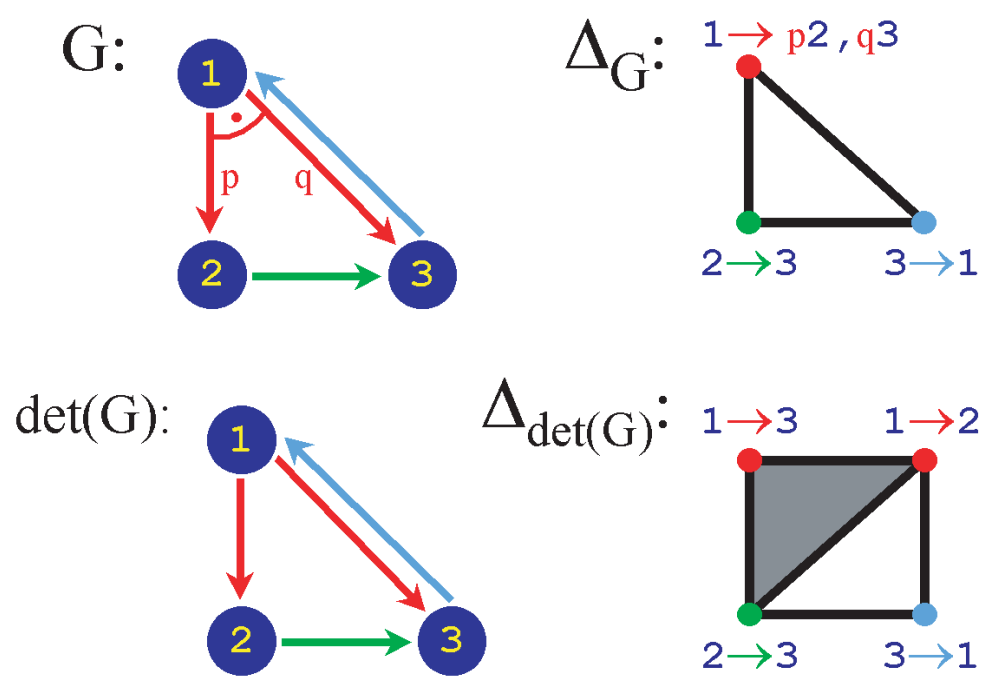

Figure 28: The top row shows a pure stochastic graph and its strategy complex. The bottom row shows the deterministic rendition of this graph plus the resulting strategy complex. Both complexes are homotopic to $S^{1}$.

Pursuing this further, we can ask where true adversity resides. The realizability theorems of Section 13 all used nondeterministic graphs, as did the $N P$-completeness result of Lemma 23. Suppose $G$ is a pure stochastic graph, in the sense that it contains only deterministic and stochastic actions but no multi-edged nondeterministic actions. Then Lemma 26 and Theorem 20 allow us to conclude that $\Delta_{G}$ is homotopic either to a sphere or to a point. Pure stochastic graphs simply cannot attain the richness of strategy complexes that nondeterministic graphs attain. Said differently, true adversity comes from nondeterministic adversaries. Stochastic adversaries can slow a system down, but do not have the ability to switch between limit cycles in the way nondeterministic adversaries sometimes can (limit cycles are the minimal nonfaces of $\bar{\Delta}_{G}$; see also the remark on page 55$)$. 


\section{Some further remarks:}

Suppose again that $G$ is a pure stochastic graph. When $\Delta_{G}$ and $\Delta_{\operatorname{det}(G)}$ are homotopic to a sphere, that sphere has dimension $n-k-1$, where $n$ is the number of states in $G$ and $k$ is the number of strongly connected components of $\operatorname{det}(G)$. In particular, if $\operatorname{det}(G)$ can be written as the disjoint union of its strongly connected components, then $\Delta_{G}$ and $\Delta_{\operatorname{det}(G)}$ will be homotopic to a sphere; otherwise, they will be contractible, as follows from [44] or Theorem 20. Observe that $k$ is no greater than the number of strongly controllable subspaces of $G$ and in fact can be less, but must be the same when $\Delta_{G}$ and $\Delta_{\operatorname{det}(G)}$ are homotopic to a sphere.

When passing to quotient graphs, let us discard any actions that become cyclic (deterministically self-looping). Then the $\operatorname{graph} \operatorname{det}(G) / \leftrightarrow$ is a directed acyclic graph. All the actions of this graph together are acyclic and thus the complex $\Delta_{\operatorname{det}(G) / \leftrightarrow}$ consists of a single simplex (all acyclic actions of $\operatorname{det}(G) / \leftrightarrow$ ) and its faces. It is empty precisely when $\operatorname{det}(G)$ can be written as the disjoint union of its strongly connected components.

The graph $G / \leftrightarrow$ is similar, yet slightly different. As we observed already, the state space of $G / \leftrightarrow$ may be larger than that of $\operatorname{det}(G) / \leftrightarrow$. With regard to strategies, as was the case for $\Delta_{\operatorname{det}(G) / \leftrightarrow,} \Delta_{G / \leftrightarrow}$ consists of a single simplex (all acyclic actions, now of $G / \leftrightarrow$ ) and its faces. A difference lies in certainty. $G / \leftrightarrow$ represents a collection of Markov chains, with all states either transient or absorbing. Whereas in $\operatorname{det}(G) / \leftrightarrow$ the system may be able to move purposefully from some state to some other state, in $G / \leftrightarrow$ it may only be able to do so with some probability; the adversary may have some stochastic choices by which to influence the outcome of motions. This is a simple Markov Decision Process [67].

\subsection{Topological Thinking in Partially Observable Spaces}

This section shows by example how the topological characterization of task solvability may help one decide whether a task has a guaranteed solution. Throughout this section all graphs are nondeterministic.

Implicitly in the analyses thus far, the system has always known its current state. If in fact the system's sensing function is imperfect, then one may redefine the system's state space so as to obtain perfect sensing in the redefined state space. The construction is straightforward $[5,54]$, albeit often with added complexity.

The nondeterministic version of this construction leads to a derived nondeterministic graph, sometimes called a knowledge space [23, 25]. A sensorless version of knowledge space appeared as the search space for the part orienter of [29].

Intuitively, the knowledge space of a graph is a new graph whose states are sets of potential robot locations consistent with the robot's sensing and action history at runtime. For completeness, we give a general definition here.

Definitions Suppose $G=(V, \mathcal{A})$ is a nondeterministic graph.

- For the purposes of the next few definitions, we assume that every action in $\mathcal{A}$ is labeled with a name, which we refer to notationally as $A$. Names of actions at a given state are unique. However, different actions at different states might have the same name. (Think of a global control with possibly different effects at different states.)

We write $A_{v}$ to mean the action in $\mathcal{A}$ whose name is $A$ and whose source is $v$, whenever such an action exists. 
- A sensor on $V$ is a covering $\Xi$ of $V$ by nonempty subsets of $V$, each of which is called a sensory interpretation set. Sensory interpretation sets may overlap.

Intuition: Whenever the system is at some state $v$, a sensor returns some value whose interpretation is a set $I \in \Xi$ such that $v \in I$. (We could model the sensor via some intermediate sensory space, but we will simply map directly to interpretations of the sensor. More general formulations of sensing appear, for instance, in [5, 54].) Sensing in our case is nondeterministic, meaning that the sensor could return any $I \in \Xi$ with $v \in I$.

Observe that $\Xi=\{V\}$ is equivalent to no sensing.

- Suppose $X \subseteq V$ and $A$ is an action name. The forward projection of $X$ under $A$, written $F_{A}(X)$, is the set of all possible locations the system might move to after executing some action named $A$, given that the system starts in $X$. In order for this definition to be sensible, the name $A$ must actually mean something at every state in $X$. Otherwise, by convention, $F_{A}(X)$ is not defined. Formally, if $A_{v}$ exists for every $v \in X$, then

$$
F_{A}(X)=\left\{u \in V \mid(v, u) \in A_{v} \text { for some } v \in X\right\} .
$$

- Once the system moves from $X$ under $A$, it can sense again. The sensor may return any sensory interpretation set $I \in \Xi$ for which $F_{A}(X) \cap I$ is not empty. If the system knows it is in set $X$ before moving under $A$, then after moving and sensing a particular $I$, the system knows it is somewhere in the set $F_{A}(X) \cap I$.

- Given $G$ and $\Xi$ as above, define a new nondeterministic graph $K=\left(V^{+}, \mathcal{A}^{+}\right)$as follows:

- $V^{+}$consists of all nonempty subsets of $V$. (Depending on $\Xi$ and $\mathcal{A}$, one may not really need to construct all of $V^{+}$, of course.)

- For every $X \in V^{+}$, and for every action name $A$ such that $A_{v}$ exists for all $v \in X$, $\mathcal{A}^{+}$contains a nondeterministic action with source $X$ given by:

$$
\left\{(X, Y) \mid \emptyset \neq Y=F_{A}(X) \bigcap I \text { for some } I \in \Xi\right\} \text {. }
$$

$K$ is the knowledge space associated with $G$ and $\Xi$.

Remark: One can define stochastic analogues of $K$ for pure stochastic graphs $G$. This leads to the so-called belief states, of great interest in Partially Observable Markov Decision Processes [54]. Some caution is advised, since problems quickly become intractable [67, 58].

\subsubsection{Inferring Task Unsolvability From Duality}

Recall Theorem 16. Failure of the theorem's contractibility condition is evidence of a potentially inescapable cycle, appearing as a minimal nonface of $\bar{\Delta}_{G}$, indicating that the graph contains no complete guaranteed strategy for attaining set $S$. We saw an example in Fig. 27.

Now consider the graph of Fig. 29, which might model a robot moving in a building. There are two corridors (states 1 and 2). In any one corridor the robot can move RIGHT or LEFT. Atriums connect the corridors at either ends. The task is to reach one particular atrium (state 3). Entry into the corridors from the other atrium (state 4) is imprecise. The gray triangle 

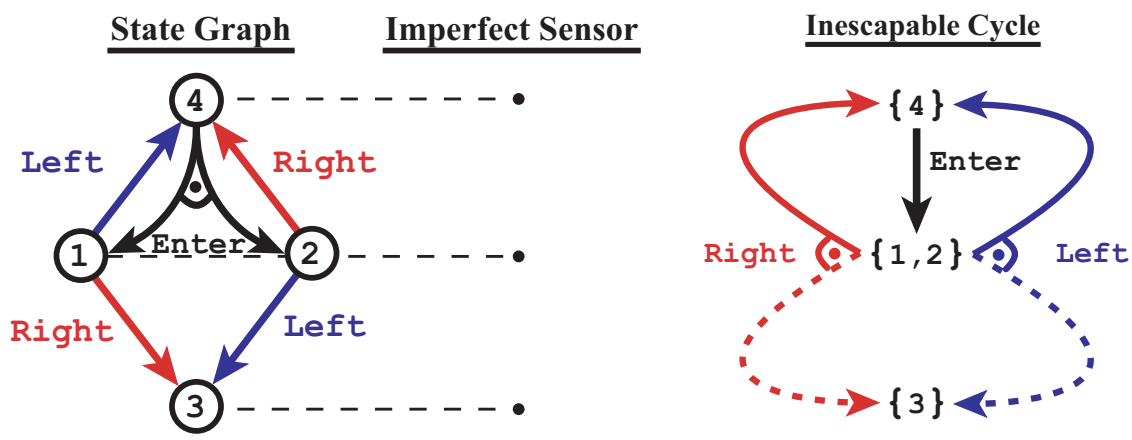

Figure 29: A graph, an imperfect sensor, and a potentially inescapable cycle between knowledge states $\{4\}$ and $\{1,2\}$ (indicated by the solid arrows in the right panel).
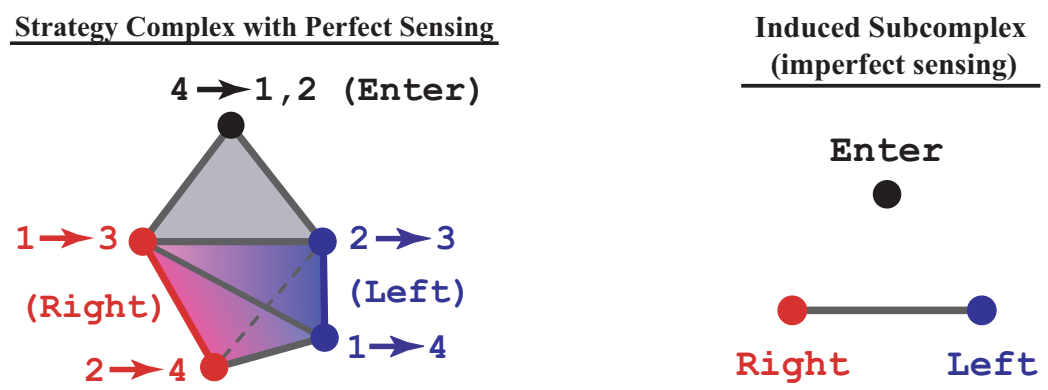

Figure 30: With perfect sensing, the strategy complex of the state graph of Fig. 29 consists of a solid triangle joined to an edge of a solid tetrahedron. With sensing ambiguity at states 1 and 2, this strategy complex collapses, inducing a non-contractible subcomplex of strategies in knowledge space that signals a potentially inescapable cycle. 
in the strategy complex of Fig. 30 constitutes a strategy for accomplishing this task, assuming perfect sensing. The strategy is to ENTER from state 4, move Right from 1, LEFT from 2.

Now imagine a robot controller unable to distinguish the two corridors (states 1 and 2) based on sensing alone. The task no longer has a guaranteed solution. One could see this in a variety of ways, for instance, by explicitly constructing the robot's knowledge space, part of which is shown in the right panel of Fig. 29. Let us take a related but more topological perspective.

One does not need to construct the knowledge space directly. Instead, one can reason about strategies. Consider the strategy complex of the original graph (left panel, Fig. 30). As we will see, this complex induces a non-contractible subcomplex of strategies in knowledge space (right panel, Fig. 30) that violates the contractibility condition of Theorem 16.

Let us focus on the actions at two key knowledge states, namely $\{4\}$, representing certainty that the system is at state 4 , and $\{1,2\}$, representing uncertainty as to whether the system is at state 1 or 2 . The tetrahedron of the original complex describes the strategies possible with perfect sensing at the graph states 1 and 2. The tetrahedron collapses to an edge under sensing ambiguity. This edge represents the two actions, RIGHT and LEFT, possible at state $\{1,2\}$ in knowledge space. In the original complex, only a portion of the tetrahedron joins with the action ENTER; action RIGHT at state 2 and action LEFT at state 1 do not join with ENTER. In the knowledge space complex, the edge $\{$ RIGHT, LEFT $\}$ consequently cannot join with the action ENTER.

We have thus exhibited a non-contractible complex describing the strategies available at a subset of knowledge space, with that subset lying in the complement of the goal (state 3). This means, no matter what the surrounding knowledge space might look like, there can exist no complete guaranteed strategy for attaining state 3 in the presence of control uncertainty at state 4 and sensing confusion between states 1 and 2 .

\subsubsection{Hypothesis-Testing and Sphere Suspension}

Definition: A suspension of a complex is another complex formed by joining each simplex of the given complex with each of two new vertices [61, 39]. For instance, the complex in Fig. 4 is a suspension of the complex in Fig. 2. The key property relevant to us currently is that the suspension of a sphere of any dimension is another sphere, of one higher dimension. For instance, (a globe of) the Earth is a suspension of the Equator by the North and South Poles.

Fig. 31 shows a variant of the example of Fig. 29. Once again there is some control uncertainty, once again the sensor cannot distinguish certain corridors. The system cannot move reliably to state 3 using pure feedback control (that is, using sensing alone, without history). For some sensor values, neither of the actions RIGHT or LEFT will make progress toward the goal 3 at all graph states consistent with the sensor value.

Fortunately, this time the confusable corridors lie in different "wings" of the building. The sensor stratifies the graph into two subgraphs, both containing the goal 3. Within each subgraph the sensor is effectively perfect and each subgraph contains a strategy guaranteed to attain the goal from anywhere within that subgraph using sensor-indexable controls that are well-defined across subgraphs.

Whenever a sensor stratifies a graph into subgraphs in this manner, there exists a hypothesis-testing strategy for attaining the goal from anywhere in the overall graph. Hypothesis-testing means: The system assumes it is in one of the subgraphs; it commands 


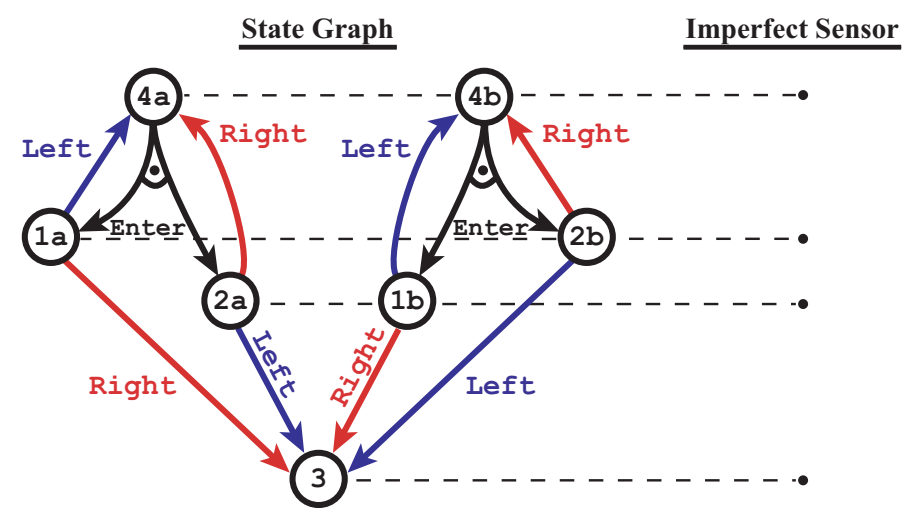

Figure 31: An imperfect sensor stratifies a graph into two subgraphs (the "a wing" and the "b wing"), over each of which sensing is effectively perfect.

actions and interprets sensor readings as if it really were in that subgraph, but also verifies consistency between predicted motions and observed sensor readings. If an inconsistency occurs, the hypothesis of being in that subgraph has been falsified and the system moves on to another hypothesis. Intuitively, this strategy eventually converges at the goal.

Hypothesis-testing is a strategy in knowledge space, but one does not need to construct knowledge space. In general, knowledge space may contain additional, possibly shorter, strategies. Fortuitously, for the example of Fig. 31, hypothesis-testing is effectively the only strategy.

\section{There is a short topological argument that hypothesis-testing converges:}

Hypothesis-testing amounts to repeated sphere suspension.

Further details: The graph $H_{i}$ describing motions under the $i^{\text {th }}$ hypothesis is equivalent to the subgraph $G_{i}$ being hypothesized, except that some actions may move nondeterministically to a new state $\perp_{i}$, signaling falsification of the hypothesis. We also add an "action" from $\perp_{i}$ to the goal, as explained below. Without loss of generality, every action of $G_{i}$, and thus $H_{i}$, contains a nondeterministic transition to the goal. (Adding these transitions does not affect the existence of strategies for attaining the goal, but focuses on the topologically significant simplices in the complexes.)

The relevant loopback complex $\Gamma_{H_{i}}$ of $H_{i}$ is then a suspension, formed by joining the relevant loopback complex $\Gamma_{G_{i}}$ of $G_{i}$ with the loopback action goal $\rightarrow \perp_{i}$ and the action $\perp_{i} \rightarrow$ goal. We now use Theorem 1 , once in each direction: Since $G_{i}$ contains a strategy guaranteed to attain the goal from any state in $G_{i}, \Gamma_{G_{i}}$ is homotopic to a sphere, of the correct dimension. Since $\Gamma_{H_{i}}$ is a suspension of $\Gamma_{G_{i}}$, it too is a sphere, again of the correct dimension, proving that hypothesis-testing converges.

What is the mysterious action $\perp_{i} \rightarrow$ goal ? It is the inductive-hypothesis that there exists a strategy for attaining the goal once the $i^{t h}$ graph-hypothesis has been falsified! (The base case is similar, except that there is no need for a state $\perp_{i}$. The final graph-hypothesis is certain.) 
Remarks: (1) One can make a very similar argument directly at the graph level, further underscoring the parallel between spheres and task solvability. That parallel shows as well that backchaining in nondeterministic graphs is much like repeated sphere suspension. (2) Hypothesis-testing is related to randomization [23, 24, 25].

\section{Conclusions}

\subsection{Summary}

The controllability structure Theorem 20 on page 51 is the most concise description of system capabilities we have currently. Implicitly, this theorem contains as special cases the full controllability Theorem 11 and the goal attainability Theorem 1. These three theorems characterize controllability of uncertain systems by the existence of spheres of a specific dimension. Theorem 16 provides a dual statement via contractibility of subcomplexes. That theorem helps one understand an adversary's capabilities in terms of forced limit cycles.

From a practical perspective, Theorem 14 on page 42 is the most useful. It suggests that when considering homotopy equivalences, one should take the homotopy simplification of a strategy complex down to the state space, and not further. That rendition provides a natural mechanism by which to compare the capabilities of different systems with a common state space, as illustrated by the design example of Sections 9 and 10.

\subsection{Other Results}

We have omitted many interesting results from this paper. The Contributions section ( $\$ 1.6)$ mentioned several. For instance, the number of nonempty simplices in the loopback complex associated with a particular goal is even if the goal is stochastically attainable and odd if it is not. This is a consequence of the Euler characteristic being even for spheres and odd for contractible spaces.

There also exist generalizations of the results presented, by reduction to them. For instance, perhaps one is interested in whether a goal is attainable from some initial subset of the state space rather than from the entire state space. One can reduce this problem to a full attainability question by modifying the graph slightly. In the nondeterministic case this is easy to see: one merely adds a nondeterministic hyperjump at each state, that jumps to every state in the initial region. These hyperjumps will never be needed by an actual strategy in the original system but serve as a convenient reduction tool.

Similarly, perhaps one is merely interested in reaching a goal with some minimum probability, but not necessarily for certain. Again, one can add hyperjumps at some states, assigning strictly positive transition times to these hyperjumps and zero transition times to all other actions. One thus reduces the original problem to the problem of attaining a goal with certainty, but now with a bound on the worst-case expected convergence times.

\subsection{Open Questions}

Our original goal was to develop a topological language for describing system capabilities. We have come a long way. Strategy and source complexes abstract away the detailed connectivity between states in a graph while retaining information about overall goal attainability. 
Consequently, one can assign topological spaces to graphs, much like labels, that succinctly summarize the graphs' capabilities.

One wonders whether this new language holds more expressive power than we have used thus far. Our key theorems are not yet completely "coordinate-free": The number of states shows up in the dimensionality of the spheres that assure controllability. Likewise, we find ourselves working with source complexes on specific state spaces, rather than more abstractly.

Hidden inside this observation is another: Our contractibility results tend to have the flavor of collapsibility (see Section 5.2). This begs the question whether the more general (and more difficult) form of contractibility is relevant, and if so, what it is telling us.

Related to this general interpretation of contractibility is the question of whether our results generalize to continuous state spaces. As we observed in the Introduction, from a practical perspective one tends to work in discrete spaces. Nonetheless, the question is interesting intellectually. For instance, imagine a sequence of finite discrete graphs that approximate ever more finely a continuous space. An amusing issue here is that finite-dimensional spheres are homotopically different from points, whereas the infinite-dimensional sphere is homotopically equivalent to a point.

\section{Acknowledgments}

Many thanks to Ben Mann for his leadership, energy, and vision, which made this research possible, enjoyable, and addictive.

This research was part of a multi-year multi-institutional effort involving topologists and roboticists working on a variety of problems at the interface of algebraic topology and autonomous systems. Many thanks to the entire "SToMP" group for their feedback and encouragement. Particular thanks to Robert Ghrist and Steven LaValle for their infectious enthusiasm and indefatigable leadership. Thanks to Robert Ghrist and Shmuel Weinberger for answering my topology questions so quickly and precisely. Thanks to Matt Mason for advice, as always, on a large number of topics. 


\section{List of Primary Symbols}

\section{Symbol}

$G, H$

V

$W$

$A, B$

$\mathcal{A}, \mathcal{B}, \mathcal{C}$

$H_{\mathcal{B}}$

$G \mid W$

$\operatorname{det}(\cdot)$

$\mathbf{R}^{n}$

$S^{1} ; S^{n-2}$

$\Sigma, \Gamma$

$\sigma, \tau, \gamma$

$\mathcal{N}(\cdot)$

$\mathcal{F}(\cdot)$

$\operatorname{sd}(\cdot)$

$\Delta_{G}$

$\bar{\Delta}_{G}$

$\bar{\Delta}_{G}^{*}$

$t_{\max }(\cdot)$

$\Delta_{G}^{T}$

$V_{\mathcal{A}}, V_{\mathcal{B}}, V_{\sigma}, V_{\tau}$

$U_{A}, U_{B}, U_{A}^{+}, U_{B}^{+}$

$[n]$

$s$

$S$

$G_{\leftarrow s}$

$\Delta_{G_{\leftarrow s}}$

$(M, W)$

$*$

$\cong$

$\approx$

$\simeq$

$\leftrightarrow$

$G / \leftrightarrow$

$G / W$

$C_{r}, \partial C_{r}, \partial C_{T+\epsilon}$
Meaning

Page Reference

nondeterministic or stochastic graph

11, 21

set of all states in a graph (state space)

11, 21

some set of states in a graph

12,21

11,20

11,21

12,23

41

64

18

$15 ; 16$

12,17

12,46

19

19

19

12, 24

42

44

24

24

22

25

16

16

30

16

16

loopback complex

22

46

17

17

17

49

49,54

49

27 


\section{List of Lemmas and Theorems}

\section{\# Description}

*1 characterization of task solvability by spheres $\quad 16$

2 Collapsibility Tool (from the literature) 19

3 Nerve Lemma (from the literature) 19

4 Quillen Fiber Lemma (from the literature) 20

5 equivalence of convergence time and Markov chain perspectives 23

6 characterization of stochastically acyclic actions 26

7 characterization of time-bounded stochastically acyclic actions $\quad 27$

8 the homotopy types of strategy complexes are given by covering sets 28

$9 \quad$ stochastically certain connectivity implies a homogeneous halfspace $\quad 31$

10 time-bounded stochastic connectivity implies an affine halfspace 31

*11 characterization of graph controllability by spheres 34

12 characterization of time-bounded graph controllability by spheres $\quad 35$

13 contractibility of start regions 41

*14 strategy and source complexes are homotopic 42

15 characterization of source complexes 42

*16 characterization of task solvability by contractibility 43

17 some conditions under which graph union implies strategy join 47

18 simplices combine if they differ by actions with the same source 47

19 controllable subspaces factor strategy complexes 50

*20 structure theorem for strategy complexes 51

21 realizability of simplicial complexes by strategy complexes 59

22 realizability of simplicial complexes as source complexes $\quad 59$

23 determining the precision of a system is $N P$-complete 60

24 small realizability is uncomputable $\quad 62$

25 detecting changes in homotopy type is uncomputable 62

26 making stochastic actions deterministic preserves homotopy type 65

* _ indicates a key theorem of the paper 


\section{List of Algorithms}

$\begin{array}{llll}\text { \# } & \text { Call Format } & \text { Description } & \text { Page } \\ 1 & \text { Backchain }(G, S) & \text { Finds actions to move off the complement of } S \text {, and repeats. } & 56 \\ 2 & \text { Strategy }(G, S) & \begin{array}{l}\text { Finds a subgraph of } G \text { with maximal state space within } \\ \text { which } S \text { is stochastically attainable. }\end{array} & 57 \\ 3 & \operatorname{Reachable}(G) & \begin{array}{l}\text { Constructs the directed graph representing stochastically } \\ \text { certain strategies for moving between states of } G .\end{array} & 57 \\ 4 & \text { Subspaces }(G) & \begin{array}{l}\text { Constructs the strongly controllable subspaces of } G . \\ -\end{array} & 58 \\ - & - & \begin{array}{l}\text { Description of an algorithm for computing quotient graphs. } \\ \text { Description of an algorithm for computing source complexes. }\end{array} & 58 \\ - & - & \text { An approach for computing strategy complexes. } & 58 \\ & & \end{array}$

\section{Term}

Page Reference

contractible

18

collapsible

18

cone

18

acyclic

12

stochastically acyclic

(nondeterministic) strategy 12

(stochastic) strategy 24

complete guaranteed strategy 16

complete stochastic strategy, stochastically attainable goal, etc. 30

System (1) 22

worst-case expected convergence times 24

an action moves off a set 41

fully controllable $\quad 35$

strongly controllable subspace 49

pure stochastic graph $\quad 65$

$\begin{array}{ll}\text { knowledge space } & 67\end{array}$ 


\section{List of Figures}

\section{Figure Description}

Page

1 nondeterministic motion 9

2 nondeterministic graph; its strategy complex 9

$3 \quad$ cyclic motions 10

4 graph with a cycle; its strategy complex 10

$5 \quad$ directed graph with three states; its strategy complex 13

$6 \quad$ graph with two cycle-inducing nondeterministic actions; its complex 13

$7 \quad$ a strongly connected graph with three states; its complex 14

8 another strongly connected graph with three states; its complex 14

$9 \quad$ loopback graph and complex for the graph of Fig. $5 \quad 15$

$10 \quad$ loopback graph and complex for the graph of Fig. $6 \quad 15$

11 homotopy equivalence favors more precise actions 20

12 stochastic graph with two eventually-convergent actions; its complex 21

13 stochastic graph with three uncertain actions; its complex 28

14 covering sets for the graph of Fig. $13 \quad 29$

15 action tuning parameters 36

$16 \quad$ snapshot of designing a system by considering covering sets 37

17 design space from graph perspective 38

18 design space from covering set perspective 39

19 design space from strategy complex perspective 39

20 the design space summarized by four simplicial complexes 40

21 source and dual complexes describing the design space 45

22 attainability versus strong controllability 49

$23 \quad$ a sample stochastic graph, perhaps modeling air travel $\quad 52$

24 nontrivial strongly controllable subspaces of the previous graph 53

$25 \quad$ quotient graph and a complete strategy for attaining one state 54

$26 \quad$ source complex of the quotient graph 54

$27 \quad$ interpretation of a minimal nonface $\quad 55$

28 deterministic instantiation of stochastic actions preserves homotopy type $\quad 65$

$\begin{array}{lll}29 & \text { imperfect sensing causes cycling } & 68\end{array}$

$30 \quad$ effect of imperfect sensing on strategy complex $\quad 68$

$\begin{array}{lll}31 & \text { an imperfect sensor stratifies a graph perfectly } & 70\end{array}$ 


\section{References}

[1] A. V. Aho, J. E. Hopcroft, and J. D. Ullman. The Design and Analysis of Computer Algorithms. Addison-Wesley, Reading, MA, 1974.

[2] S. Akella and M. T. Mason. Posing polygonal objects in the plane by pushing. Proc. IEEE Intl. Conference on Robotics and Automation, pages 2255-2262, 1992.

[3] A. Barr, P. Cohen, and E. Feigenbaum, editors. The Handbook of Artificial Intelligence. William Kaufmann, Inc., Los Altos, California, 1981-1989.

[4] R. Bellman. Dynamic Programming. Princeton University Press, Princeton, N.J., 1957.

[5] D. P. Bertsekas. Dynamic Programming: Deterministic and Stochastic Models. PrenticeHall, Englewood Cliffs, N.J., 1987.

[6] A. Björner. Topological methods. In R. Graham, M. Grötschel, and L. Lovász, editors, Handbook of Combinatorics, volume II, pages 1819-1872. North-Holland, Amsterdam, 1995.

[7] A. Björner and M. Tancer. Note: Combinatorial Alexander duality - A short and elementary proof. Discrete and Computational Geometry, 42(4):586-593, 2009.

[8] A. Björner and V. Welker. Complexes of directed graphs. SIAM J. Discrete Math, 12(4):413-424, 1999.

[9] M. Brady, J. M. Hollerbach, T. Johnson, T. Lozano-Pérez, and M. T. Mason. Robot Motion: Planning and Control. MIT Press, Cambridge, MA, 1982.

[10] R. C. Brost. Automatic grasp planning in the presence of uncertainty. Intl. J. Robotics Research, 7(1):3-17, 1988.

[11] R. C. Brost and K. Y. Goldberg. A complete algorithm for designing planar fixtures using modular components. IEEE Trans. on Robotics and Automation, 12(1):31-46, 1996.

[12] J. F. Canny. The Complexity of Robot Motion Planning. MIT Press, Cambridge, MA, 1988.

[13] V. de Silva and R. Ghrist. Coordinate-free coverage in sensor networks with controlled boundaries via homology. Intl. J. Robotics Research, 25(12):1205-1222, 2006.

[14] B. R. Donald. Robot motion planning with uncertainty. Artificial Intelligence, 37(13):223-271, 1988.

[15] B. R. Donald. Error Detection and Recovery in Robotics. Lecture Notes in Computer Science, No. 336. Springer-Verlag, Berlin, 1989.

[16] B. R. Donald. Planning multi-step error detection and recovery strategies. Intl. J. Robotics Research, 9(1):3-60, 1990.

[17] B. R. Donald. On information invariants in robotics. Artificial Intelligence, 72:217-304, 1995. 
[18] B. R. Donald and J. Jennings. Constructive recognizability for task-directed robot programming. Robotics and Autonomous Systems, 9:41-74, 1992.

[19] B. R. Donald, J. Jennings, and D. Rus. Towards a theory of information invariants for cooperating autonomous mobile robots. Sixth International Symposium on Robotics Research, pages 29-48, 1993.

[20] J. Dugundji. Topology. Allyn and Bacon, Boston, 1966.

[21] H. F. Durrant-Whyte. Sensor models and multisensor integration. Intl. J. Robotics Research, 7(6):97-113, 1988.

[22] M. A. Erdmann. Using backprojections for fine motion planning with uncertainty. Intl. J. Robotics Research, 5(1):19-45, 1986.

[23] M. A. Erdmann. On Probabilistic Strategies for Robot Tasks. PhD thesis, EECS, MIT, Cambridge, MA, 1989. Also available as Technical Report AI-TR-1155.

[24] M. A. Erdmann. Randomization in robot tasks. Intl. J. Robotics Research, 11(5):399-436, 1992.

[25] M. A. Erdmann. Randomization for robot tasks: Using dynamic programming in the space of knowledge states. Algorithmica, 10(2-4):248-291, 1993.

[26] M. A. Erdmann. Understanding action and sensing by designing action-based sensors. Intl. J. Robotics Research, 14(5):483-509, 1995.

[27] M. A. Erdmann. An exploration of nonprehensile two-palm manipulation. Intl. J. Robotics Research, 17(5):485-503, 1998.

[28] M. A. Erdmann. On the topology of plans. Eighth Intl. Workshop on the Algorithmic Foundations of Robotics, Guanajuato, Mexico, December 2008.

[29] M. A. Erdmann and M. T. Mason. An exploration of sensorless manipulation. IEEE J. Robotics and Automation, 4(4):369-379, 1988.

[30] M. A. Erdmann, M. T. Mason, and G. Vaněček, Jr. Mechanical parts orienting: The case of a polyhedron on a table. Algorithmica, 10(2-4):226-247, 1993.

[31] M. Farber. Topological complexity of motion planning. Discrete and Computational Geometry, 29(2):211-221, 2003.

[32] W. Feller. An Introduction to Probability Theory and Its Applications. Volume I. John Wiley and Sons, New York, third edition, 1968.

[33] A. Fox and S. Hutchinson. Exploiting visual constraints in the synthesis of uncertaintytolerant motion plans. IEEE Trans. on Robotics and Automation, 11(1):56-71, 1995.

[34] R. Ghrist and S. LaValle. Nonpositive curvature and Pareto-optimal coordination of robots. SIAM J. Control \& Opt, 45(5):1697-1713, 2006. 
[35] R. Ghrist, J. O'Kane, and S. LaValle. Computing Pareto optimal coordinations on roadmaps. Intl. J. Robotics Research, 24(11):997-1010, 2005.

[36] R. Ghrist and V. Peterson. The geometry and topology of reconfiguration. Adv. Appl. Math, 38:302-323, 2007.

[37] K. Y. Goldberg. Orienting polygonal parts without sensors. Algorithmica, 10(2-4):201$225,1993$.

[38] L. Guilamo, B. Tovar, and S. LaValle. Pursuit-evasion in an unknown environment using gap navigation trees. Proc. IEEE/RSJ Intl. Conference on Intelligent Robots and Systems, pages 3456-3462, 2004.

[39] A. Hatcher. Algebraic Topology. Cambridge University Press, Cambridge, 2002.

[40] M. Herlihy and N. Shavit. The topological structure of asynchronous computability. J. ACM, 46(6):858-923, 1999.

[41] J. Hopcroft and G. Wilfong. Motion of objects in contact. Intl. J. Robotics Research, 4(4):32-46, 1986.

[42] K. Hsiao, L. Kaelbling, and T. Lozano-Pérez. Grasping POMDPs. Proc. IEEE Intl. Conference on Robotics and Automation, pages 4685-4692, 2007.

[43] K. Hsiao, L. Kaelbling, and T. Lozano-Pérez. Robust belief-based execution of manipulation programs. Eighth Intl. Workshop on the Algorithmic Foundations of Robotics, Guanajuato, Mexico, December 2008.

[44] A. Hultman. Directed subgraph complexes. Elec. J. Combinatorics, 11(1):R75, 2004.

[45] Y.-B. Jia and M. A. Erdmann. Geometric sensing of known planar shapes. Intl. J. Robotics Research, 15(4):365-392, 1996.

[46] Y.-B. Jia and M. A. Erdmann. Pose and motion from contact. Intl. J. Robotics Research, 18(5):466-490, 1999.

[47] J. Jonsson. Simplicial Complexes of Graphs. PhD thesis, Department of Mathematics, KTH, Stockholm, Sweden, 2005.

[48] S. Karlin and H. M. Taylor. A Second Course in Stochastic Processes. Academic Press, New York, 1981.

[49] R. M. Karp. Reducibility among combinatorial problems. In R. E. Miller and J. W. Thatcher, editors, Complexity of Computer Computations, pages 85-103. Plenum Press, New York, 1972.

[50] J. L. Kelley. General Topology. Springer Verlag, New York, 1955.

[51] J. Kerr and B. Roth. Analysis of multifingered hands. Intl. J. Robotics Research, 4(4):3-17, 1986. 
[52] D. E. Koditschek. An approach to autonomous robot assembly. Robotica, 12(2):137-155, 1994.

[53] J.-C. Latombe. Robot Motion Planning. Kluwer Academic Publishers, Boston, 1991.

[54] S. M. LaValle. Planning Algorithms. Cambridge University Press, New York, 2006.

[55] A. Lazanas and J.-C. Latombe. Motion planning with uncertainty: A landmark approach. Artificial Intelligence, 76(1-2):285-317, 1995.

[56] T. Lozano-Pérez, M. Mason, and R. Taylor. Automatic synthesis of fine-motion strategies for robots. Intl. J. Robotics Research, 3(1):3-24, 1984.

[57] D. Mackenzie. ROBOTICS: Topologists and Roboticists Explore an 'Inchoate World'. Science, 301(5634):756, 8 August 2003.

[58] O. Madani, S. Hanks, and A. Condon. On the undecidability of probabilistic planning and related stochastic optimization problems. Artificial Intelligence, 147(1-2):5-34, 2003.

[59] M. T. Mason. Automatic planning of fine motions: Correctness and completeness. Proc. IEEE Intl. Conference on Robotics and Automation, pages 492-503, 1984.

[60] M. T. Mason. Mechanics and planning of manipulator pushing operations. Intl. J. Robotics Research, 5(3):53-71, 1986.

[61] J. R. Munkres. Elements of Algebraic Topology. Addison-Wesley, Menlo Park, 1984.

[62] J. Nevins, D. Whitney, S. Drake, D. Killoran, M. Lynch, D. Seltzer, S. Simunovic, R. Spencer, P. Watson, and A. Woodin. Exploratory research in industrial modular assembly. Technical Report R-921, The Charles Stark Draper Laboratory, 1975.

[63] P. S. Novikov. On the algorithmic unsolvability of the word problem in group theory. Trudy Mat. Inst. Steklov, 44:1-143, 1955.

[64] S. P. Novikov. Appendix to: The problem of the algorithmic discrimination of the standard three-dimensional sphere, by I. A. Volodin, V. E. Kuznetsov, and A. T. Fomenko. Uspekhi Mat. Nauk, 29(5):71-168, 1974.

[65] J. M. O'Kane and S. M. LaValle. Comparing the power of robots. Intl. J. Robotics Research, 27(1):5-23, 2008.

[66] C. H. Papadimitriou and K. Steiglitz. Combinatorial Optimization: Algorithms and Complexity. Prentice-Hall, Englewood Cliffs, N.J., 1982.

[67] C. H. Papadimitriou and J. N. Tsitsiklis. The complexity of Markov decision processes. Mathematics of Operations Research, 12(3):441-450, 1987.

[68] D. Quillen. Homotopy properties of the poset of non-trivial $p$-subgroups of a group. Advances in Math., 28:101-128, 1978.

[69] J. J. Rotman. An Introduction to Algebraic Topology. Springer Verlag, New York, 1988. 
[70] R. C. Smith and P. Cheeseman. On the representation and estimation of spatial uncertainty. Intl. J. Robotics Research, 5(4):56-68, 1986.

[71] E. H. Spanier. Algebraic Topology. McGraw-Hill, San Francisco, 1966.

[72] R. H. Taylor, M. T. Mason, and K. Y. Goldberg. Sensor-based manipulation planning as a game with nature. In R. Bolles and B. Roth, editors, Robotics Research, volume IV, pages 421-429. MIT Press, Cambridge, MA, 1988.

[73] B. Tovar, R. Murrieta, and S. LaValle. Distance-optimal navigation in an unknown environment without sensing distances. IEEE Transactions on Robotics, 23(3):506-518, 2007.

[74] B. Tovar, A. Yershova, J. O'Kane, and S. LaValle. Information spaces for mobile robots. Proc. Intl. Workshop on Robot Motion and Control, 2005.

[75] M. L. Wachs. Poset topology: Tools and applications. Technical report, IAS/Park City Mathematics Institute, Summer 2004.

[76] S. Weinberger. Computers, Rigidity, and Moduli: The Large-Scale Fractal Geometry of Riemannian Moduli Space. M. B. Porter Lectures. Princeton University Press, Princeton, 2004 .

[77] D. E. Whitney. Quasi-static assembly of compliantly supported rigid parts. Journal of Dynamic Systems, Measurement, and Control, 104(1):65-77, 1982.

[78] Wikipedia, The Free Encyclopedia. Markov Decision Process. http://en.wikipedia.org/wiki/Markov_decision_process. 